\title{
Über die Verteilung der Primzahlen in Folgen der Form $[f(n+x)]$
}

von

FRTEDRICH ROESLER (München)

Einleitung. Dirichlets Prinizahlsatz

$$
\lim _{s \downarrow 1}\left(\log \frac{1}{s-1}\right)^{-1} \sum_{\substack{p \operatorname{Primzahl} \\ p=(k)}} p^{-s}=\frac{1}{\varphi(k)}
$$

läßt sich mit dem Taubersatz von Hardy-Littlewood-Karamata umformen in die asymptotische Dichte-Aussage

$$
\lim _{x \rightarrow \infty} \frac{\sum_{1 \leq n \leqslant N} \frac{\chi_{P}(f(n))}{n}}{\sum_{1 \leqslant n \leqslant N^{N}} \frac{\chi_{P}(n)}{n}}=\frac{k}{\varphi(k)},
$$

in der $\chi_{P}$ die charakteristische Funktion der Menge $P$ aller Primzahlen bezeichnet und $f(y)=7 y+l$ Polynomfunktion in $\mathbb{Z}[y]$ ist mit $(k, l)=1$ und positivem Leitkoeffizienten $k$. Formel (1) bildet den Ausgangspunkt der folgenden Überlegungen.

Ist $\not{P}$ irgendeine fest rorgegebene unendliche Teilmenge der natürlichen Zahlen $\boldsymbol{N}$ und $f$ eine beliebige Funktion von $\boldsymbol{R}_{+}=[0, \infty[$ in $\boldsymbol{R}$, so erweist sich die Wahl geeigneter Wichtungsfunktionen für die Summanden $\chi_{\mathscr{P}}(f(n))$ und $\chi_{\mathscr{G}}(n)$ in einem Ausdruck analog zur linken Seite von (1) als abhängig rom Wachstum

(i) der Anzahlfunktion $\pi_{\mathscr{P}}(y)=\sum_{0<m \leqslant y} \chi_{\mathscr{P} P}(m)$ dex Menge $\mathscr{P}$,

(ii) des Fehlerterms $\pi_{2}(y)$, wenn $\dot{\pi}_{\mathscr{P}}(y)$ mit einer handhabbaren Funktion $\pi_{1}(y)$ approximiert wird dureh $\pi_{\mathscr{P}}(y)=\pi_{1}(y)+O\left(\pi_{2}(y)\right)$,

(iii) der Funktion $f$.

Allgemein soll deshalb zunächst für beliebige monoton wachsende Funktionen $\psi_{1}$ und $\psi_{2}$ von $\boldsymbol{R}_{+}$in $\boldsymbol{R}_{+}$und für jedes $x$ aus dem halboffenen 
Intervail

$$
I^{1}=[0, I[
$$

die (asymptotische) $\psi_{1}-\psi_{\mathrm{s}}$-Dichte $D_{\psi_{2}}^{\mu_{1}}(\mathscr{P}, f)(x)$ der Menge $\mathscr{P}$ in der Folge der ganzen Zahlen $[f(n+n)]_{n \in N}$ definiert werden durch

mit

$$
D_{\psi_{2}}^{\eta_{1}}(\mathscr{P}, f)(x)=\lim _{N \rightarrow \infty} D_{\psi_{2}}^{\eta_{1}}(\mathscr{P}, f ; N)(x)
$$

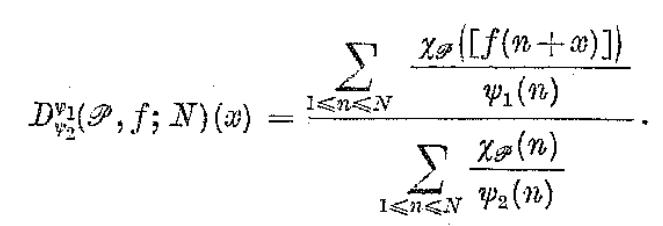

Bemerkng 3 in Teil 7 zeigt an einem Beispiel, daß der Limes in (2) durehaus nicht immer existiert.

Folgende Spezialisierungen werden wir im einzelnen behandeln:

(i) $\psi_{1}(y)=\psi_{2}(y)=1$. Dam heißt $D_{\psi_{2}}^{y_{1}}$ die natürliche Dichte von $\mathscr{P}$ in $[f(n+\infty)]_{n \in N}$. Sie soll hier mit $D_{\text {har }}^{\text {nat }}$ bezeichnet werden.

(ii) $\psi_{1}(y)=\psi_{2}(y)=y$. In diesem Fall soll $D_{312}^{\varphi_{1}}$ die harmonisehe Dichte von $\mathscr{P}$ in $[f(n+\infty)]_{n \in N}$ heißen and mit $D_{\text {har }}^{\text {nat }}$ abgekürzt werlen. In der Literatur (etrra bei Ostmann [23], S. 95) wird sie zuweilen logarithmische Dichte genannt. Diesen Ausdruck wollen wir aber reservieren für

(iii) $\psi_{1}(y)=\max \left\{\log 2, \log y_{j}, \psi_{2}(y)=y \cdot D_{\psi_{2}}^{\psi_{1}}\right.$ nennen wir dann die logarithmisch-harmonische Dichte und schreiben $D_{\text {har }}^{\mathrm{log}}$.

Mit (1) folgt nun fiur $f(y)=k y+l$ mit natürlichem $k$ und reellem $l$ und für jedes $x$ ans $I^{1}$

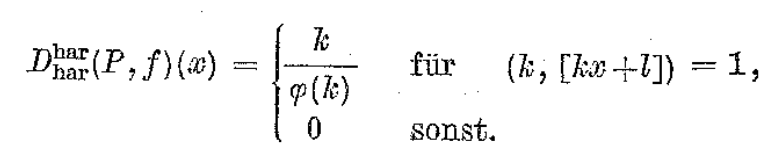

Aus der genaueren Aussage über die Verteilung der Primzahlen in arithmetischen Progressionen von de la Vallée-Poussin folgt, daß (3) richtig bleibt, wenn darin $D_{\text {lar }}^{\text {har }}$ ersetzt wird durch die präzisere Dichtefunktion $D_{\text {nat. }}^{\text {nat }}$ Hat der Leitkoeffizient $k$ ron $f$ die Form $r / s$ mit natürlichen, teilerfremden Zabilen $r$ und $s$, so ergibt sich bei Untersuchung der Folgen $\left[\frac{r}{s}(s n+m+x)+l\right]_{n \in \mathbb{N}}$

$$
D_{\mathrm{nat}}^{\mathrm{nat}}(P, f)(x)=k \frac{\Phi(x)}{\varphi(r)}
$$

Wobei $\Phi(x)$ die Anzahl der ganzen Zahlen $m$ mit $0 \leqslant m<s$ bezeichnet, für die $[k(m+x)+l]$ und $r$ teilerfremd sind.
Für irmationales $k>1$ und beliebiges reelles $l$ hat Fogels in [7], Theorem 1, mit Abschätzungen ron trigonometrischen Summen für die Anzahl $x(N ; k, l)$ der Primzahlen $\leqslant N$ in der Folge $[k n+l]_{n \in N}$ die Formel $\pi(N ; z, l) \sim \frac{1}{k} \frac{X}{\log X}$ bewiesen, wenn die Quotienten $z_{c_{0}}, k_{1}, k_{2}, \ldots$ in der Kettenbruchentwicklung ron $k_{i}$ der Bedingung $k_{n}$ $=O\left(e^{c^{n}}\right)$ mit geeigneten $c>1$ genügen. Wit einem Satz yon Borel-Bernstein (Hardy-Wright [12], Theorem 197) folgt daraus für $f(y)=k y+l$ aus $\boldsymbol{R}[y]$ mit irrationalem $k>1$

$$
D_{\text {nat }}^{\text {nat }}(P, f)(x)=1 \quad \text { für fast alle } \mathfrak{a} \text { aus } I^{1} .
$$

Dasselbe Ergebnis findet sich auch bei Leitmann-Tolke [20].

Über die Primzahlverteilung in den Folgen $[f(n+x)]_{n \in \mathbf{N}}$ im nichtlinearen Fall sind Ergebnisse nur für Funktionen der Form $f_{k ; a, \beta}(y)$ $=\alpha y^{k}+\beta$ veröffentlicht: Deshouillers [32] und Nordon [33] haben bewiesen, dab für fast alle $l_{i}>1$ und fast alle $a>0$ die Folgen $\left[n^{k}\right]_{n \in N}$ und $\left[a n^{2}\right]_{n \in N}$ je unendlich viele Primzahlen darstellen. Darüberhinaus haben sie $D_{\text {nat }}^{\text {nat }}\left(\mathscr{P}, f_{h ; 1,0}\right)(0)$ und $D_{\text {nat }}^{\text {nat }}\left(\mathscr{P}, f_{2 ; a, 0}\right)(0)$ nach oben abgeschätzt. Zuvor schon war gezeigt worden:

$$
D_{\mathrm{na} \hat{\mathrm{t}}}^{\mathrm{nat}}\left(\mathscr{P} ; f_{k ; 1,0}\right)(0)=1 / \hbar
$$

and zwar für $1<k<12 / 11$ von Pjateckii-Shapiro [25], für $1<k<10 / 9$ von Kolesnik [17] und für fast alle $k$ aus ] 1, 2[ ron Leitmann-Wolke [20]. Fïr Primzahlen in arithmetischen Progressionen $\mathscr{P}_{q, l}=\{p \in \mathscr{P} ; p \equiv l(q)\}$, $(l, q)=1$, hat Rieger $[26]$ Abschätzangen von $D_{\mathrm{nat}}^{\text {nat }}\left(\mathscr{P}_{q, l}, f_{k ; a, \beta} ; N\right)(0)$ angegeben, und zwar nach oben für $1<k<2$ und nach unten für 1 $<k<12 / 11$. Leitmann and Wolke haben in [19] das Ergebnis von Pjateckii-Shapiro auf arithmetische Progressionen übertragen:

$$
D_{\text {nat }}^{\text {nat }}\left(\mathscr{P}_{q, l}, f_{k ; 1,0}\right)(0)=1 / k \quad \text { für } \quad 1<7<12 / 11 .
$$

Ferner sind für ganzwertige Polpnomfunktionen $f$ Absehätzungen von $D_{\text {nat }}^{\text {nat }}(P, f ; N)(0)$ nach oben unter Benutzung ron Siebmethoden bewiesen worden; ein derartiges Ergebnis wird zu Anfang von Teil 6 zitiert.

Es werden im folgenden zwei Klassen nichtlinearer reeller Funktionen untersucht, and zwar rerallgemeinerte Polynomfunktionen und Exponentialfunktionen

$$
\begin{aligned}
& \mathscr{F}_{\mathrm{pol}}=\left\{a y^{k t}+\sum_{i=1}^{m} a_{i} y^{k_{i}} ; a>0, k>k_{1}>\ldots>k_{m} \geqslant 0\right\}, \\
& \mathscr{F}_{\mathrm{exp}}=\left\{e^{k y+t}+f(y) ; k>0, f \in \mathscr{F}_{\mathrm{poI}} \cup\{0\}\right\} .
\end{aligned}
$$

Für jedes $f$ aus einer dieser Klassen wird $k$ der Grad ron $f$ genannt. Die 
Wahl gerade dieser Klassen ist historisch motiviert. Aus den Beweisen der Arbeit läßt sich jedoch. leicht ersehen, für welche größeren Funktionsklassen dieselben Aussagen xichtig bleiben. Entscheidend in die Beweise der Sätze geht nämlich neben. Differenzierbarkeitsbedingungen an $f$ nur die Wachstumsordnung der Funktionen ein.

Das Hanptergebnis dieser Arbeit besagt (Satz 11):

(i) Ist $f$ aus $\mathscr{F}_{\text {exp }}$ vom Grad $k$, so gilt für die logarithmisch-harmonische Dichte der Primzahlen in der Folge $[f(n+x)]_{n \in \boldsymbol{N}}$

$$
D_{\text {har }}^{\mathrm{log}}(P, f)(x)=\frac{1}{k} \quad \text { für fast alle } x \text { ans } I^{1} \text {. }
$$

(ii) Unter der Voraussetzung, daß der Primzahlsatz

$$
\pi_{P}(y)=\operatorname{li} y+O(\sqrt{y} \log y)
$$

richtig ist, gilt für die harmonische Diehte der Primzallen in der Folge $[f(n+x)]_{n \in \mathbf{N}}$ für Funktionen $f$ ans $\mathscr{F}$ pol mit Grad $k>2$

$$
D_{\text {har }}^{\text {har }}(P, f)(x)=\frac{1}{k} \quad \text { für fast alle } x \text { aus } I^{1} \text {. }
$$

Der in (ii) voransgesetzte Primzahlsatz

$$
\pi_{P}(y)=\operatorname{li} y+O(\sqrt{y} \log y)
$$

ist äquivalent zur Richtigkeit der Riemannschen Vermutung (Davenport [4], S. 117f). Zum Beweis von (i) hingegen genügt die Benutzung des bekannten Primzahlsatzes

$$
\pi_{P}(y)=\operatorname{li} y+O\left(\frac{y}{\exp \sqrt{\log y}}\right) .
$$

Teil 1 behandelt qualitative Überlegungen im Zusammenhang mit der Verteilung der Zahlen einer Menge $\mathscr{P}$ aus $\boldsymbol{N}$ in Folgen der Form $[f(n+x)]_{n \in \mathbf{N}}$. In Teil 2 werden drei Integralabschätzungen (Lemmata 1, 2 und 3) bewiesen, die mehrfach den Ausgangspunkt für die Ableitung quantitativer Dichteaussagen bilden werden. Anschließend läßt sich Einblick gewinnen in die Beziehung zwischen den beiden. Wiehtungsfunktionen $\psi_{1}$ und $\psi_{2}$. Als erste Anwendang wird in Teil 3 ein Satz über die Verteilung der "Psendoprimzahlen" $P^{*}=\left\{\left[\mathrm{li}^{-1} n\right] ; n \geqslant 2\right\}$ in den Folgen $[f(n+x)]_{n \in \boldsymbol{N}}$ bewiesen (Satz 4). Dasselbe Resultat ist auch für die Primzahlmenge $P$ selbst zu erwarten $\div$ und findet sich in fast der. selben Form dann in Satz 11. Der Beweis für $P$ statt $P^{*}$ erweist sich jedoch als ungleich komplizierter. Das liegt daran, daB die Zahlen aus $P$ unregelmäBiger verteilt sind als die aus $P^{*}$. Der Unweg, der für die Funktionen aus $\mathscr{F}_{\text {pol }}$ deshalb eingeschlagen wird, erstreckt sich über die Teile 4,5 und 6 und besteht darin, zunächst Kenntnisse zu sammeln über dje Teilbarkeit der Folgenelemente von $[f(n+x)]_{n \in \mathbf{N}}$ durch eine rorgegebene Zahl $d$ - also $D(d \boldsymbol{N}, f)$ zu ermitteln - und diese dann mit dem SelbergSieb umzusetzen in eine qualitatice Aussage über die Primzahlverteilung in dieser Folge (Teil 6, Hilfssatz 4(i)). Es liegt in der Natur des SelbergSiebes, da:B dieses Vorgehen nicht mehr liefert als Abschätzungen nach oben in der richtigen Größenordnung; daß dies aber für den Beweis der quantitativen Aussage in Satz 11 genügt, erklärt sich daraus, daß das Sieb-Ergebnis aus Teil $6 \mathrm{im}$ Beweis von Satz 11 nur zur Abschätzung eines 0 -Termes herangezogen werden $\mathrm{muB}$; die quantitative Aussage hingegen basiert auf Lemma. 2. Die aus den Lemmata 1 und 3 direkt bereisbare Gleichung $D_{\text {har }}^{\operatorname{har}}(d N, f)=1$ für Funktionen aus $\mathscr{F}_{\text {pol }}$ (Satz $6(\mathrm{i})$ fürr $\mathscr{P}=d \boldsymbol{N}$ ) reicht indessen nicht aus zur Anwendung des Selberg-Siebes. Dies und das deshalb notwendige Vorgehen in den Teilen 4 und 5 rersuchen die Bemerkungen am Sehlu $\beta$ von Teil $3 \mathrm{zu}$ motivieren.

Teil 4 behandelt eine sehr anschauliche Frage aus der Theorie der diophantischen Approximationen: Gegeben ist eine Schnur mit $N$ Knoten, je zwei aufeinanderfolgende im festen Abstand $y$ voneinander. Diese Schnur wird auf eine Spule vom Umfang 1 gerollt. Wie groß wird dann der maximale Bogenabstand benachbarter Knoten? Die Antwort liefert Satz 7. Damit läßt sich ein Integral abschätzen (Satz 8), mit dessen Hilfe in Teil $\tilde{s}$ eine Aussage über $D_{\text {nat }}^{\text {nat }}(d \boldsymbol{N}, f)$ für Funktionen aus $\mathscr{F}_{\text {pol }}$ gewonnen wiid (Satz 9), und zwar mit einem O-Term, der den Anforderungen des Selberg-Siebes genügt. In Teil 6 wird das Siebverfahren durchgeführt in Anlehnung an die Darstellungen bei Halberstam-Roth [10], Kap. IV und Schwarz [27], Kap. IV. Dabei ergibt sich ein weiteres Ergebnis (Satz 10) über die Verteilung der Primzahlen in den Folgen $[f(n+x)]_{n \in \mathbf{N}}$.

$\varphi \quad$ ist durchweg die Euler-Funktion;

$\mu$ bezeichnet das Lebesgue-Ma:B auf $\boldsymbol{R}$ und an einer Stelle in Teil 6 auch die Möbiussche Funktion.

$f=g$ heißt, daß $f(y)=g(y)$ für alle $y$ außerhalb einer Lebesgue-Nullmonge richtig ist.

$f(y) \leqslant g(y)+O(h(y))$ steht ablürzend dafür, daß mit einer Konstanten $c>0$ für alle großen $y$ die Ungleichung $f(y) \leqslant g(y)+c|h(y)|$ gilt, und

$f(y) \geqslant g(y)+O(h(y))$ meint $f(y) \geqslant g(y)-c \mid h(y)]$.

$\Theta$ mit oder ohne Index wird stets für Zahlen aus $[0,1]$ verwendet.

[y] für reelles $y$ bezeichnet den Ganzteil und

$\{y\}=y-[y]$ den Bruchteil von $y$.

$\mathrm{A}\left(Y ;\left(y_{n}\right)_{1 \leqslant n \leqslant N}\right)$ für Mengen $Y$ reeller Zahlen und endliche Folgen $\left(y_{n}\right)$ sei die Anzahl der Elemente $y_{n}$ in $Y$. 
$\exp (y)$ steht zuweilen für $e^{y}$,

li $(y)$ ist der Integrallogarithmus, und

$\delta_{i j}$ bezeichnet das Kronecker-Symbol.

Herm Professor A. Leubbecher danke ich sehr herzlich für seine Anregungen und das fortwährende Interesse, das er dieser Arbeit entgegengebracht hat. Weiter gilt mein Dank Herrn H.-W. Kirstein und Dr. H. L. Skudlarek für frnchtbare Diskussionen über den Gegenstand der Arbeit.

1. Qualitative Aussagen. $\mathscr{P}$ bezeichne stets eine unendliche Teilmenge der natïrlichen Zahlen. Neben der Menge $P$ aller Primzahlen werden für $\mathscr{P}$ auftreten die eingangs erwähnten Pseudoprimzallen $P^{*}$ und die Vielfachen $d N$ von natürlichen Zahlen $d$. Gegenstand der Untersuchungen ist für zweimal differenzierbare Funktionen $f: \boldsymbol{R}_{+} \rightarrow \boldsymbol{R}$ aus zunächst nicht näher spezifizierten Funktionsklassen $\mathscr{F}$ die Verteilung der Zahlen aus $\mathscr{P}$ innerhalb der Folgen $[f(n+\infty)]_{n \in \mathbf{N}}$ mit $x$ aus $I^{1}$. Dazu definieren wir zu vorgegebenen $\mathscr{P}, f$ aus $\mathscr{F}$ und einer monoton steigenden Wichtungsfunktion $\varphi: \boldsymbol{R}_{+} \rightarrow \boldsymbol{R}_{+}$

$$
\begin{array}{cc}
s_{n}: I^{1} \rightarrow\{0,1\}, & s_{n}(x)=\chi_{\mathcal{P}}([f(n+x)]), \\
S_{N}^{q}: I^{1} \rightarrow \boldsymbol{R}_{+}, & S_{N}^{\psi}(x)=\sum_{n=1}^{N} \frac{s_{n}(x)}{\psi(n)} .
\end{array}
$$

Ist nicht eindeutig ersichtlich, um welche Menge $\mathscr{P}$ es sich handelt, wird ausfübrlicher $S_{N}^{p}(\mathscr{P} ; x)$ geschrieben. $S_{N}^{\text {p}}(x)$ gibt die mit $\psi$ gewichtete "Anzahl" der Zahlen aus $\mathscr{P}$ in der endhichen Folge $[f(n+x)]_{1 \leqslant n \leqslant N}$ an. Die speziellen Wichtungen

$$
\psi(y)=1, \quad \psi(y)=y, \quad \psi(y)=\max \{\log 2, \log y\}
$$

kennzeichnen wir suggestiv durch $S_{N}^{\text {nat }}, S_{N}^{\text {har }}, S_{N}^{\text {log }}$.

Aus rechnerischen Gründen erweist es sich als sinnvoll, statt der Mengen $\mathscr{F}$ jeweils zunächst nur die Teilmengen

$$
\mathscr{F}^{*}=\{f \in \mathscr{F} ; f \text { erfüllt }(\mathrm{F} 1)\}
$$

mit der Bedingung

(F1) $f(0) \geqslant 1, \quad f^{\prime}(0) \geqslant 1, \quad f^{\prime \prime}(0)>0, \quad f^{\prime \prime}$ monoton wachsend

zu untersuchen.

Es sei also $f$ aus $\mathscr{F}^{*}$. [a, $b\left[\right.$ bezeichne ein halboffenes Intervall in $\boldsymbol{I}^{\mathbf{1}}$ und $n$ eine feste natürliche Zahl. Für $x$ aus $[a, b[$ ist $[f(n+x)]$ Element von $\mathscr{P}$ genau dann, wenn $x$ in eines der Intervalle

$$
I_{p, n}=\left[f^{-1}(p)-n, f^{-1}(p+1)-n[, \quad p \in \mathscr{P},\right.
$$

und damit in eines der

$$
I_{p, n}^{a, b}=I_{p, n} \cap[a, b[, \quad p \in \mathscr{P},
$$

fällt. $I_{p, n}^{a, b}$ ist nicht lexr nur für die $p$ aus

$$
\mathscr{P}_{n}(a, b)=\{p \in \mathscr{P} ; f(n+a)-1<p<f(n+b)\} .
$$

$s_{n}$ nimmt auf $[a, b[$ also den Wert 1 an genau tür die $x$ aus einem der paarweise disjunkten Intervalle $I_{p, n}^{a, b}, p \in \mathscr{P}_{n}(a, b)$. Ferner ist $I_{p, n}^{a, b}=I_{p, n}$ für genand die $p$ aus

$$
\mathscr{P}_{n}^{*}(a, b)=\{p \in \not{P} ; f(n+a) \leqslant p \leqslant f(n+b)-1\}
$$

und.

$$
\mathscr{P}_{n}^{* *}(a, b)=\mathscr{P}_{n}(a, b)-\mathscr{P}_{n}^{*}(\dot{a}, b)
$$

enthält höchstens zrej Elemente.

Tur Analyse der Funktionen $s_{n}$ ist die Länge, also das Lebesgue-Mai3 $\mu$ der Intervalle $I_{p, n}^{a, b}$ zu ermitteln: Ans den Differenzierbarkeits- und Monotonieforderungen (F1) an $f$ ergibt sich mit dem Mittelwertsatz

$\begin{array}{ll}\text { (1) } \mu\left(I_{p, n}^{a, b}\right)=\mu\left(I_{p, n}\right)=\frac{1}{f^{\prime}\left(n+\Theta_{j}\right)}, a \leqslant \Theta_{p} \leqslant b, & \text { für } p \in \mathscr{P}_{n}^{*}(a, b), \\ \text { (2) } \mu\left(I_{p, n}^{a, b}\right) \leqslant \frac{1}{f^{\prime}(n+a)} & \text { für } p \in \mathscr{P}_{n}^{* *}(a, b) .\end{array}$

SATz 1. $f: \boldsymbol{R}_{+} \rightarrow \boldsymbol{R}$ sei zweimal differenzierbar mit für $y \geqslant y_{0}$ monoton wachsender zweiter Ableitung und $f^{\prime \prime}\left(y_{0}\right)>0$. Dann sind gleichbedeutend:

(i) Fïr alle Zahlen $a<b$ in $[0,1]$ sehneidet die Intervallfolge $[f(n+a)$, $f(n+b)-1]_{n \in \mathrm{N}}$ die Henge $\mathscr{P}$ unendlich of

(ii) Jede nichtleere offene Menge in $[0,1]$ enthält einen Punkt $x$, für den die Folge $[f(n+x)]_{n \in \mathbf{N}}$ unendlich viele Zahlen aus $\mathscr{P}$ darstellt.

(iii) Jede nichtleere offene Blenge in $[0,1]$ enthäth uberabähhlbar viele Punkte $x$, für welehe die Folgen $[f(n+x)]_{n \in N}$ je unendlich viele Zahlen aus $\not{P}$ darstellen.

Beweis. Es darf $f \in \mathscr{F}^{*}$ vorausgesetzt werden, denn sicher genügt $f_{M}(y)=f(M I+y)$ für hinreichend großes natürliches $M$ der Bedingung (F1), und sind die Äquivalenzen richtig für $f_{M}$, so auch für $f$.

(ii) folgt aus (iii) ohne Miuhe. Zum Beweis von (i) aus (ii) sei $a<a<b$ und $\left(p_{m}\right)_{n \in N}$ eine streng monoton wachsende Folge von Zahlen aus $\mathscr{P}$ die in $[f(n+x)]_{n \in \mathbf{N}}$ auftreten. Für alle hinreichend großen $m$ ist dann zunächist $c \in I_{p_{m}, n_{m}}$ mit $n_{m}=\left[f^{-1}\left(p_{m}\right)\right]$ und wegen $\lim _{m \rightarrow \infty} \mu\left(I_{p_{m}, n_{m}}\right)=0$ sogar $I_{p_{m}, n_{m}} \subset[a, b]$, also $p_{m} \in\left[f\left(n_{m}+a\right), f\left(n_{m}+b\right)-1\right]$. 
Zum Beweis von (iii) aus (i) sei eine nichtlecre offene Menge in $[0,1]$ vorgegeben. Diese enthält ein Intervall $[a, b]$ mit $a<b$. Wir konstruieren eine injektive Abbildung $\Phi$ von der Menge $\mathfrak{A}$ aller Folgen $A=\left(a_{m}\right)_{m \in N}$ mit Werten $a_{m}$ aus $\{0,1\}$ in die Menge allex $x$ aus $[a, b]$, für die $[f(n+x)]_{n \in N}$ unendlich viele Zahlen aus $\mathscr{P}$ darstellt. Fürr $p$ aus $\mathscr{P}$ sei $n_{p}=\left[f^{-1}(p)\right]$ und

$$
J_{p}^{i}=\left[f^{-1}\left(p+\frac{i}{2}\right)-n_{p}, f^{-1}\left(p+\frac{2 i+1}{4}\right)-n_{p}\right], \quad i=0,1
$$

Die $J_{p}^{i}$ sind abgeschlossen, disjunkt und in $I_{p, n_{p}}$ enthalten. Gemäß (i) existiert ein kleinstes $p_{0}$ in $\mathscr{P}$ mit $I_{p_{0},{ }^{n} p_{0}} \subset[a, b]$. Ist nun $A$ aus $\mathfrak{A}$ vorgelegt und sind $p_{1}, \ldots, p_{m-1}$ schon bestimmt, so kann $p_{m}$ wegen (i) definiert werden als die kleinste $\mathrm{Zahl}$ in $\not \supset$ mit

$$
I_{p_{m}, n_{p_{m}}} \subset J_{p_{m-1}}^{a_{m}}
$$

Die Folge $\left(p_{m}\right)_{m \in \boldsymbol{N}}$ steigt streng monoton, da $\mu\left(I_{p, n}\right)$ für wachsendes $p$ monoton fällt.

$$
\Phi(A)=\bigcap_{m \in N} J_{p_{m-1}}^{a_{m}}
$$

definiert einen Punkt in $[a, b]$, denn aus (3) folgt $J_{p_{m}+1}^{a_{m+1}} \subset J_{x_{m-1}}^{a_{m}}$, und ferner ist $\lim \mu\left(J_{p_{m}}^{a_{m+1}}\right)=0$. Also stellt die Folge $[f(n+\Phi(A))]_{n \in \mathbf{N}}$ alle $p_{m}$ dar. Sind $A$ und $A^{\prime}$ verschiedene Folgen in $\mathfrak{A}$ und ist $m$ der kleinste Index mit $a_{m} \neq a_{m}^{\prime}$, so folgt $p_{k}=p_{k}^{\prime}$ für $0 \leqslant k<m$ und damit $\Phi(A) \in J_{p_{m}}^{a_{m}}$, $\Phi\left(A^{\prime}\right) \in J_{p_{m-1}^{\prime}}^{a_{m}}$. Da $J_{p_{m-1}}^{a_{m}}$ und $J_{p_{m-1}}^{a_{m}}$ disjunkt sind, ist $\Phi(A) \neq \Phi\left(A^{\prime}\right)$, und das beweist die Injektivität von $\Phi$.

CoRolLAR. Zu jedem $f$ ans $\mathscr{F}_{\text {pol }}$ vom Grad $k>12 / 5$ ist die Menge der $x$ aus $I^{1}$, für welche die Folge $[f(n+x)]_{n \in N}$ unendlich viele Primzahlen darstellt, überabzählbar und dicht in $I^{1}$.

Bemerkung. Ist die Vermutung von Oramér [3] richtig, daß es zu jedem himreichend großen $y$ eine Primzahl zwischen $y$ und $y+O\left(\log ^{2} y\right)$ gibt, so gilt die Aussage des Corollars für alle Funktionen aus $\mathscr{F}_{\text {pol }}$ vom Grad $k>1$.

Beweis. Ohne Einschränkung darf $f \in \mathscr{F}_{\text {pol }}^{*}$ vorausgesetzt werden; ferner seien Zahlen $a<b$ in $[0,1]$ vorgegeben. Wegen $k>12 / 5$ existiert ein $\varepsilon>0$ derart, daß für alle hinreichend großen $n$

$$
f(n+a)^{7 / 12+8}+1<(b-a) f^{\prime}(n+a)
$$

ist. Das zusammen mit einem Satz von Ingham [14] - in der derzeit besten numerischen Fassung findet er sich bei Huxley. [13] -, demzufolge für jedes $a>7 / 12$ and genügend großes $y$ zwischen $y$ und $y+y^{\alpha}$ stets eine Primzahl liegt, garantiert zu jedem $n \geqslant n_{0}$ die Existenz einer Primzahl $p_{n}$ mit

$$
f(n+a) \leqslant p_{n} \leqslant f(n+a)+f(n+a)^{7 / 2+e} \leqslant f(n+b)-1
$$

Also ist (i) in Satz 1 richtig; und aus (iii) folgt die Aussage des Corollars. Es hat sich also gezeigt, daß fïr $P=P$ und himreichend stark wachsende Funktionen aus $\mathscr{F}_{\text {pol }}$ zu jedem $n$ aus $N$ und $p$ aus $\mathscr{P}_{n}^{*}(0,1)$ ein $n^{\prime}>n$ und ein $p^{\prime}$ aus $\mathscr{P}_{n^{\prime}}^{*}(0,1)$ existieren mit $I_{p^{\prime}, n^{\prime}} \subset I_{p, n^{*}}$. Durch eine zusätzliche Forderung an das Wachstum von $f$ läßt sich $n^{\prime}=n+1$ erzwingen:

SATZ 2. Erfüllt $f$ die Toraussetzungen von Satz 1 und ist überdies

$$
f(y)^{\alpha}=O\left(\frac{f^{\prime}(y)}{f^{\prime}(y-1)}\right) \text { für ein } a>\frac{7}{12}
$$

so gibt es ein $n_{t}$ und überabähthlbar viele $x$ aus $I^{1}$ derart, daß die Folgen $[f(n+x)]_{n \geqslant n_{f}}$ nur ans Primaahlen bestehen.

Beweis. Wieder darf $f \in \mathscr{F} *$ vorausgesetzt werden. Ferner reicht es zu zeigen, daß für jedes $n \geqslant n_{0}, i \in\{0,1\}$ und $p \in \mathscr{P}_{n}^{*}(0,1)$ ein $p^{\prime} \in P$ existiert mit $I_{p^{\prime}, n+1} \subset J_{p}^{i}$. Dann folgt die Behauptung des Satzes auf die gleiche Weise wie die ron (iii) aus (i) in Satz 1. Die zweite Bedingung an $f$ garantiert mit einem $\left.\alpha^{\prime} \in\right] \pi / 12, \alpha\left[\right.$ für alle $y \geqslant y_{1}$

$$
f(y+1)^{a^{\prime}} \leqslant \frac{1}{4} \frac{f^{\prime}(y+1)}{f^{\prime}(y)}
$$

also

$$
f\left(f^{-1}(y) \div 1\right)^{a^{\prime}} \leqslant f\left(f^{-1}(y+\theta)+1\right)^{\alpha^{\prime}} \leqslant \frac{1}{4} \frac{f^{\prime}\left(f^{-1}(y+\theta)+1\right)}{f^{\prime} f^{-1}(y+\Theta)}
$$

für alle $y \geqslant y_{2}$ und $\Theta \geqslant 0$. Der Nittelwertsatz, angewandt auf $h(y)$ $=f\left(f^{-1}(y)+1\right)$, liefert für geeignetes $\Theta_{y} \geqslant 0$

$$
f\left(f^{-1}\left(y+\frac{1}{4}\right)+1\right)-f\left(f^{-1}(y)+1\right)=\frac{1}{4} \frac{f^{\prime}\left(f^{-1}\left(y+\Theta_{y}\right)+1\right)}{f^{\prime} f^{-1}\left(y+\Theta_{y}\right)} .
$$

Zusammen mit (4) und einem $\left.\alpha^{\prime \prime} \in\right] 7 / 12, \alpha^{\prime}[$ führt das auf

$$
f\left(f^{-1}(y)+1\right)+f\left(f^{-1}(y)+1\right)^{\alpha^{\prime \prime}} \leqslant f\left(f^{-1}\left(y+\frac{1}{2}\right)+1\right)-1
$$

für alle $y \geqslant y_{3}$. Also liegt für jedes $y \geqslant y_{4}$ zwischen $f\left(f^{-1}(y)+1\right)$ und $f\left(f^{-1}\left(y+\frac{1}{4}\right)+1\right)-1$ eine Primzahl. Ist nun $n_{0} \geqslant f^{-1}\left(y_{4}\right), \quad n_{z} \geqslant n_{0}$ and $p \in \mathscr{P}_{n}^{*}(0,1)$, so folgt wegen $n=\left[f^{-1}(p)\right]$ für $y=p+i / 2$ zunächst $y \geqslant y_{4}$ and damit die Existenz einer Primzahl $p^{\prime}$ zwischen $f\left(f^{-1}\left(p+\frac{i}{2}\right)+1\right)$ and $f\left(f^{-1}\left(p+\frac{2 i+1}{4}\right)+1\right)-1$. Das heißt aber

$$
\left[f^{-1}\left(p^{\prime}\right), f^{-1}\left(p^{\prime}+1\right)\left[\subset \left[f^{-1}\left(p+\frac{i}{2}\right)+1, f^{-1}\left(p+\frac{2 i+1}{4}\right)+1[,\right.\right.\right.
$$

also $I_{p^{\prime}, n+1} \subset J_{p}^{i}$. 
CoRoLLAR. Zn jedem $b>12 / 5$ existieren überabyählbar viele $a>1$ derart, daß die Folge $\left[c^{b^{n}}\right\rceil_{n \in \mathbf{N}}$ mur Primzahlen darstellt.

Beweis. $f(y)=c_{0}^{b y}$ exfüllt für $c_{0}>1$ und $b>12 / 5$ die Voraussetzungen von Satz 2. Deshalb gibt es ein $n_{0}$ und überabzählbar viele $x$ aus $I^{1}$, für welche die Folge $\left[c_{0}^{b^{n+x}}\right]_{n>n_{0}}$, also $\left[c(x)^{b^{n}}\right]_{n \in \mathbf{N}}$ mit $c(x)=c_{0}^{b_{0}^{n_{0}}+x}$, nur Primzahlen darstellt.

Bemerkungen. (1) Für $b=3$ ist die Existenz einer Zahl $a$ mit der im Corollar beschriebenen Eigenschaft ein Ergebnis von Mills [21]. Kuipers [18] hat gezeigt, daß zn jeder natiullichen Zahl $b \geqslant 3$ ein $c$ existiert, mit dem die Folge $\left[c^{b^{n}}\right]_{n \in N}$ nur Primzahlen darstellt, und Ansari [1] hat dasselbe für jedes $b>77 / 29$ bewiesen. Zusammengefabt sind diese Ergebnisse bei Dudley [6]; dort wird auch der Zusammenhang mit der Riemannschen $\zeta$-Funktion diskutiert: Ist die Vermutung richtig, daß $\zeta\left(\frac{1}{2}+i t\right)$ $=O\left(t^{\varepsilon}\right)$ für jedes positive $\varepsilon$ gilt, so stimmt Ansaris Satz für jedes $b>2$.

(2) Bei Niven [22] findet sich ein ähnlicher Satz: Zu jedem $c>1$ existieren mindestens abzählbar vicle $b>1$, für welche die Folgen $\left[c^{b}\right]_{n \in \mathbf{N}}$ nur Primzahlen darstellen.

(3) Nach Wright [31] sind sowohl für jedes $b>8 / 3$ die Menge aller $c>1$ als auch für jedes $c>1$ die Nenge aller $b>1$, für die $\left[c^{b^{n}}\right]_{n \in N}$ nur Primzahlen darstellt, üburabzählbare, nirgends dichte Nullmengen. Corollar 1 zu Satz 3 wird zeigen, daß für jedes $b>1$ sogar die Menge aller $c>1$, für die $\left[e^{b^{n}}\right]_{n \in N}$ lediglich unendlich viele Primzahlen enthält, Nullmenge ist.

Für $f$ aus $\mathscr{F}^{*}$ wird nun das Integral $\int_{0}^{1} \mathcal{S}_{N}^{\text {nat }}(\mathscr{P} ; x) d x$ untersucht. Dabei ist neben Formel (1) die Gleichung

$$
\mu\left(I_{p, n}^{0,1}\right)+\mu\left(I_{p, n+1}^{\beta, 1}\right)=\mu\left(I_{p, n}\right)
$$

für $p$ aus $\mathscr{P}_{n}(0,1) \cap \mathscr{\mathscr { F }}_{n+1}(0,1)$ zu beachten.

$$
\begin{aligned}
& \int_{0}^{1} S_{N}^{\text {nat }}(\mathscr{P} ; x) d x=\sum_{n=1}^{N} \int_{0}^{1} s_{n}(x) d n=\sum_{n=1}^{N} \sum_{p \in \mathscr{P}_{P_{0}}(0,1)} \mu\left(I_{p, n}^{0,1}\right) \\
& =\sum_{\substack{p \in \vartheta^{j} \\
f(0) \leqslant p \leqslant f(N)}} \mu\left(I_{p, n}\right)+O(1)=\sum_{\substack{p \in \mathscr{F}^{\prime} \\
f(0) \leqslant p \leqslant f(N)}}\left(f^{-1}\right)^{\prime}\left(p+\Theta_{p}\right)+O(1) \\
& =\sum_{\substack{p \in \xi^{*} \\
p \leqslant f(N)}}\left(f^{-1}\right)^{\prime}(p)+O(1),
\end{aligned}
$$

wobei in der letzten Summe nur über diejenigen $p$ aus $\not P$ mit $p \leqslant f(N)$ summiert wird, für die $\left(f^{-1}\right)^{\prime}(p)$ definiert ist.

Die Teile 2 bis 7 dieser Arbeit behandeln den Fall

$$
\sum_{p}^{*}\left(f^{-1}\right)^{\prime}(p)=\infty
$$

Hat hingegen die Summe einen endlichen Wert $c$, so läßt sich der Satz von Levi anwenden : Die Treppenfunktionen $S_{N}^{\text {nat }}\left(\mathscr{g}^{\not} ; \mathfrak{c}\right)$ bilden eine monoton steigende Folge mit durch $c+O(1)$ beschränkter Integralfolge. Also ist $\lim _{N \rightarrow \infty} S_{N}^{\text {nat }}(\mathscr{P} ; x)=\infty$ höchstens auf einer Nollmenge. Mit anderen Worten:

SATZ 3. Erfullt $f$ die Voraussetzungen von Satz 1 und ist

$$
\sum_{p \in \mathbb{P}}^{*}\left(f^{-1}\right)^{\prime}(p)<\infty
$$

so stellen fïr fast alle $n \geqslant 0$ die Frotgen $[f(n+\infty)]_{n=\mathbf{N}}$ je nur endlich viele Zahler ans $\mathscr{P}$ dar.

Ist zum Beispiel $\mathscr{P}$ die Menge der Quadratzahlen und $f$ eine vexallgemeinerte Polynomfunktion rom Grad $k>2$, so gilt $\sum_{m \in N}\left(f^{-1}\right)^{\prime}\left(m^{2}\right)<\infty$, and $[f(n+x)]_{n \in N}$ enthält für fast jedes $x$ nur endlich viele Quadrate.

COROLLAR 1. Für jedes $b>1$ stellen für fast alle $c>1$ die Folgen $\left[c^{b^{n}}\right]_{n \in \mathbf{N}}$ je nur endlich viele Primzahlen dar.

Im Binblick auf die Fermat-Zahlen sei noch erwähnt:

ConolLaR 2. Die positiven Zahlen c, für die $\left[\left(2^{2^{n}}\right)^{c}+1\right]_{n \in \mathrm{N}}$ unendlich viele Primzahlen enthälẗ, bilden eine dichte, überabsählbare Nullmenge in $\boldsymbol{R}_{+}$.

Beweis der Corollare. Es sei $f_{i}(y)=a^{b^{y}+} \delta_{i, 2}$, und $i$ bezeichne die Nummer des Corollars. Dann ist

$$
f_{i}^{\prime}(y)=(\log a)(\log b) b^{y} a^{b^{y}}
$$

und.

$$
f_{i}^{-1}(y)=\frac{1}{\log b}\left(\log \log \left(y-\delta_{i, 2}\right)-\log \log a\right)
$$

Aus dem Primzalilsatz folgt $p_{n} \sim n \log n$, das zeigt für $n \geqslant n_{0}$ und passendes $C>0$

$$
f_{i}^{\prime} f_{i}^{-1}\left(p_{n}\right)=(\log a)(\log b) \frac{\log \left(p_{n}-\delta_{i, 2}\right)}{\log a}\left(p_{n}-\delta_{i, 2}\right) \geqslant C n \log ^{2} n
$$

und $\sum_{n \geqslant 2}\left(n \log ^{2} n\right)^{-1}$ ist endlich.

Also bilden die $x$ aus $I^{1}$, für die $\left[\left(a^{b^{x}}\right)^{b^{n}}+\delta_{i, 2}\right]_{n \in \mathbf{N}}$ unendlich viele Primzahlen darstellt, eine Nullmenge $M$. Daraus folgt erstens, daß auch die Menge der $c$ aus $\left[a, a^{b}\left[\right.\right.$ mit $c=a^{b^{x}}$ und $w$ aus $M$ Nullmenge ist, and Überdeckung von $] 1, \infty\left[\right.$ mit abzählbar vielen Intervallen $\left[a, a^{b}[\right.$ bei festem $b$ liefert die Aussage von Corollar 1 . Zweitens folgt daraus mit $a=b$ $=2$, daß zu jedem ganzzahligen $m$ die $x$ aus $I^{1}$, für die $\left[\left(2^{2^{n}}\right)^{2^{m+x}}+1\right]_{n \in \mathbb{N}}$ unendlich viele Primzahlen darstellt, das Maß 0 haben. Die Menge der $c$ in Corollar 2 ist also Nullmenge. Daß diese Menge überabzählbar und dicht in $\boldsymbol{R}_{+}$ist, folgt aus Satz 1 durch Nachweis der Eigensehaft (i) für $f_{m}(y)$ 
$=2^{2^{y+m}}+1:$ Bertiands Postulat zeigt, daß für $a<b$ und hinreichend großes $n$ zwischen $f_{m}(n+a)$ und $f_{m}(n+b)-1$ stets eine Primzahl liegt.

2. Drei Integralabschätzungen. Wir beginnen mit einer Reihe von Forderungen an die Menge $\mathscr{P}$ : Die Anzahlfunktion

$$
\pi_{\mathscr{P}}(y)=\sum_{0<m \leqslant y} \chi_{\mathscr{P}}(m)
$$

zu $\mathscr{P}$ soll sich approximieren lassen durch $\pi_{1}(y)$ mit einem Fehler $\pi_{2}(y)$ $=0\left(\pi_{1}(y)\right) ;$ also

$$
\pi_{\mathscr{P}}(y)=\pi_{1}(y)+O\left(\pi_{2}(y)\right) .
$$

Dabei seien $\pi_{1}, \pi_{2}: \boldsymbol{R}_{+} \rightarrow \boldsymbol{R}_{+}$zweimal differenzierbar. Für die folgenden Rechnungen werden Monotonieeigenschaften von $\pi_{1}$ und $\pi_{2}$ und Abschätzungen der Form $\pi_{2}(C y)=O_{C}\left(\pi_{2}(y)\right)$ für positives $O$ benötigt; wir fordern deshallo

$$
x_{i}^{\prime}(y) \geqslant 0, \quad x_{i}^{\prime \prime}(y) \leqslant 0 \quad(i=1,2) \quad \text { für } \quad y>1 .
$$

Ferner erweist es sich als notwendig - und Satz 3 hat das schon angedeutet, die Mengen $\mathscr{F}^{*}$ durch Ausschluß allzu stark wachsender Funktionen, genauer, durch die Forderung

(F2)

zu verkleinern $\mathrm{zu}$

$$
f^{(i)}(y+\Theta)=O_{f}\left(f^{(i)}(y)\right) \quad(i=0,1,2)
$$

$$
\mathscr{F}^{* * *}=\left\{f \in \mathscr{F}^{*} ; f \text { erfüllt }(\mathrm{F} 2)\right\} .
$$

Jedes $f$ aus $\mathscr{F}^{* *}$ genügt dann einer Gleichung $f^{\prime \prime}(y)=O_{f}\left(f^{\prime}(y)\right)$. Für $\mathscr{F}_{\text {pol }}^{*}$ und $\mathscr{F}_{\text {exp }}^{*}$ ist (F2) belanglos.

Die Forderungen an $\pi_{1}$ und $\pi_{2} \xi$ statten es, zu $f$ aus $\mathscr{F}^{* *}$ die Anzahl der $p$ aus $\mathscr{P}_{n}(a, b)$ mit dem Mittelwertsatz abzuschätzen durch

$$
\begin{aligned}
\left|\mathscr{P}_{n}(a, b)\right|= & \pi_{\mathscr{P}}(f(n+b))-\pi_{\mathscr{F}}(f(n+a))+O(1) \\
= & \pi_{1} f(n+b)-\pi_{1} f(n+a)+O\left(\pi_{2} f(n+b)\right) \\
= & \left.(b-a) f^{\prime}\left(n+\Theta_{n}\right) \pi_{1}^{\prime} f\left(n+\Theta_{n}\right)+O_{f}\left(\pi_{2} f(n)\right), \quad \Theta_{n} \in\right] a, b[ \\
= & (b-a) f^{\prime}(n+a) \pi_{1}^{\prime} f\left(n+\Theta_{n}\right)+ \\
& \quad+O_{f}\left(\pi_{2} f(n)+(b-a)^{2} f^{\prime \prime}(n) \pi_{1}^{\prime} f(n)\right) .
\end{aligned}
$$

Für die Länge der Intervalle $I_{p, n}^{a, b}$ werden wir die Abschätzung

(2) $\quad \mu\left(I_{x, n}^{a, b}\right)=\frac{1}{f^{\prime}(n+a)}+O_{f}\left((b-a) \frac{f^{\prime \prime}(n)}{f^{\prime}(n)^{2}}\right), \quad p \in \mathscr{P}_{n}^{*}(a, b)$

benutzen. Sie folgt aus Formel (1) in Teil 1 unter Beachtung von

$$
0 \leqslant \frac{1}{f^{\prime}(n+a)}-\frac{1}{f^{\prime}(n+b)}=\langle b-a) \frac{f^{\prime \prime}(n+\Theta)}{f^{\prime}(n+\Theta)^{2}}=O_{f}\left((b-a) \frac{f^{\prime \prime}(n)}{f^{\prime}(n)^{2}}\right)
$$

Als erstes soll zu $f$ aus $\mathscr{F}^{* *}$ das Integral $\int_{a}^{b} S_{N}^{w}(\mathscr{P} ; x) d a$ abgeschätzt werden. Die Konsequenz $f^{\prime \prime}(y)=O_{f}\left(f^{\prime}(y)\right)$ aus (F2), die Voraussetzangen über $\pi_{1}$ und $\pi_{2}$ sowie die Formeln $(1)$ und $(2)$ zeigen

$$
\text { (3) } \begin{aligned}
\int_{a}^{b} s_{n}(x) d x= & \sum_{p \subseteq \mathscr{S}_{n}(a, b)} \mu\left(I_{n, n}^{a, b}\right) \\
= & \left((b-a) f^{\prime}(n+a) \pi_{1}^{\prime} f\left(n+\Theta_{n}\right)+O_{f}\left(\pi_{\mathrm{g}} f(n)+\right.\right. \\
& \left.\left.+(b-a)^{2} f^{\prime \prime}(n) \pi_{1}^{\prime} f(n)\right)\right)\left(\frac{1}{f^{\prime}(n+a)}+O_{f}\left((b-a) \frac{f^{\prime \prime}(n)}{f^{\prime}(n)^{2}}\right)\right) \\
= & (b-a) \pi_{1}^{\prime} f\left(n+\Theta_{n}\right)+O_{f}\left(\frac{\pi_{2} f(n)}{f^{\prime}(n)}+(b-a)^{2} \frac{f^{\prime \prime}(n)}{f^{\prime}(n)} \pi_{1}^{\prime} f(n)\right) .
\end{aligned}
$$

Daraus ergibt sich

$$
\begin{aligned}
\int_{a}^{b} S_{N}^{w}(x) d x= & \sum_{n=1}^{N} \frac{1}{\psi(n)} \int_{a}^{b} s_{n}(x) d x \\
= & (b-a) \sum_{n=1}^{N} \frac{\pi_{1}^{\prime} f\left(n+\Theta_{n}\right)}{\psi(n)}+ \\
& +O_{f}\left(\sum_{n=1}^{N} \frac{\pi_{2} f(n)}{f^{\prime}(n) \psi(n)}+(b-a)^{2} \sum_{n=1}^{N} \frac{f^{\prime \prime}(n) \pi_{1}^{\prime} f(n)}{f^{\prime}(n) \psi(n)}\right)
\end{aligned}
$$

Weil nach Voraussetzung $\pi_{1}^{\prime}$ und $1 / \psi$ monoton fallen, läßt sich der Hauptterm abschätzen durch

$$
\sum_{n=1}^{N} \frac{\pi_{1}^{\prime} f(n)}{\psi(n)} \geqslant \sum_{n=1}^{N} \frac{\pi_{1}^{\prime} f\left(n+\Theta_{n}\right)}{\psi(n)} \geqslant \sum_{n=1}^{N} \frac{\pi_{1}^{\prime} f(n+1)}{\psi(n+1)}=\sum_{n=1}^{N} \frac{\pi_{1}^{\prime} f^{\prime}(n)}{\psi(n)}+O_{f}(1) .
$$

Das beweist

LEivcua 1. Für f aus $\mathscr{F}^{* *}$ und $0 \leqslant a<b \leqslant 1$ ist

$$
\int_{a}^{b} S_{N}^{\varphi}(\mathscr{P} ; x) d x=(b-a) \Pi(N)+O_{f}\left(R_{1}(a, b ; N)\right)
$$

mit

$$
\begin{gathered}
\Pi(N)=\sum_{n=1}^{N} \frac{\pi_{1}^{\prime}(f(n))}{\psi(n)}, \\
R_{1}(a, b ; N)=(b-a)^{2} R_{11}(N)+R_{12}(N) \\
R_{11}(N)=\sum_{n=1}^{N} \frac{f^{\prime \prime}(n) \pi_{1}^{\prime}(f(n))}{f^{\prime}(n) \psi(n)}, \quad R_{12}(N)=\sum_{n=1}^{N} \frac{\pi_{2}(f(n))}{f^{\prime}(n) \psi(n)}
\end{gathered}
$$


Als zweites Integral wird nntersucht

$$
\int_{a}^{b} S_{M}^{\mathrm{nat}}(\mathscr{P} ; x) S_{N}^{\mathrm{mat}}(\mathscr{P} ; x) d x=\sum_{\substack{1 \leqslant m \leqslant M \\ 1 \leqslant n \leqslant N}} \int_{a}^{b} s_{m}(x) s_{n}(x) d x
$$

Betrachten wir zunächst die einzeInen Summanden rechts in (4): Anf genau $\left|\mathscr{P}_{m}(a, b)\right|$ Intervallen $J$ innerhalb $\left[a, b\left[\right.\right.$ nimmt $s_{m}(x)$ den Wert 1 an, und die Länge jedes dieser Intervalle ist nach (1), (2) in Teil 1 höchstens $\frac{1}{f^{\prime}(m+a)}$. Die Integrale $\int_{J} s_{n}(x) d x$ lassen sich deshalb mit Formel (3) abschätzen, wenn darin rechts $b-a$ ersetzt wird durch $\frac{1}{f^{\prime}(m+a)}$.

Mit Formel (1) für $\left|\mathscr{P}_{m}(a, b)\right|$ ergibt sich

$$
\begin{aligned}
& \int_{a}^{b} s_{m}(x) s_{n}(x) d x \leqslant\left((b-a) f^{\prime}(m+a) \pi_{1}^{\prime} f(m)+o_{f}\left(\pi_{2} f(m)+\right.\right. \\
& \left.\left.+(b-a)^{2} f^{\prime \prime}(m) \pi_{1}^{\prime} f(m)\right)\right)\left(\frac{\pi_{1}^{\prime} f(n)}{f^{\prime}(m+a)}+o_{f}\left(\frac{\pi_{2} f(n)}{f^{\prime}(n)}+\frac{f^{\prime \prime}(n) \pi_{1}^{\prime} f(n)}{f^{\prime}(n) f^{\prime}(m)^{2}}\right)\right) \\
& \leqslant(b-a) \pi_{1}^{\prime} f(m) \pi_{1}^{\prime} f(n)+O_{f}\left(\sum_{i=1}^{8} T_{i}\right)
\end{aligned}
$$

mit den 0 -Termen

$$
\begin{aligned}
& T_{1}=(b-a) \frac{f^{\prime}(m)}{f^{\prime}(n)} \pi_{1}^{\prime} f(m) \pi_{2} f(n), \\
& T_{2}=(b-a) \frac{f^{\prime \prime}(n)}{f^{\prime}(n) f^{\prime}(m)} \pi_{1}^{\prime} f(m) \pi_{1}^{\prime} f(n), \\
& T_{8}=\frac{1}{f^{\prime}(m)} \pi_{1}^{\prime} f(n) \pi_{2} f(m), \\
& T_{4}=\frac{1}{f^{\prime}(n)} \pi_{2} f(m) \pi_{2} f(n), \\
& T_{5}=\frac{f^{\prime \prime}(n)}{f^{\prime}(n) f^{\prime}(m)^{2}} \pi_{1}^{\prime} f(n) \pi_{2} f(m), \\
& T_{6}=(b-a)^{2} \frac{f^{\prime \prime}(m)}{f^{\prime}(m)} \pi_{1}^{\prime} f(m) \pi_{1}^{\prime} f(n), \\
& T_{1}=(b-a)^{2} \frac{f^{\prime \prime}(m)}{f^{\prime}(n)} \pi_{1}^{\prime} f(m) \pi_{2} f(n), \\
& T_{8}=(b-a)^{2} \frac{f^{\prime \prime}(m) f^{\prime \prime}(n)}{f^{\prime}(m)^{2} f^{\prime}(n)} \pi_{1}^{\prime} f(m) \pi_{1}^{\prime} f(n),
\end{aligned}
$$

Als in den Anwendungen kritischster Term wird sich $T_{1}$ erweisen. Nach Multiplikation mit geeigneten positiven Konstanten wird $T_{8}$ durch $T_{6}$ majorisiert, $T_{7}$ durch $T_{1}$ and $T_{5}$ wie auch $T_{3}$ durch $T_{3}$; letzteres, weil $\pi_{1}^{\prime}$ fällt, während $\tau_{2}$ steigt. Übrig bleibt

(5) $\quad \int_{a}^{b} s_{m}(x) s_{n}(x) d x \leqslant(b-a) \pi_{1}^{\prime} f(m) \pi_{1}^{\prime} f(n)+O_{f}\left(T_{1}+T_{3}+T_{4}+T_{6}\right)$,

and das wird in (1) eingesetzt:

$$
\begin{aligned}
\int_{a}^{b} S_{M I}^{\text {nat }}(x) S_{N}^{\text {nat }}(x) d x \leqslant(b-a) & \sum_{\substack{1 \leqslant n+M \\
1 \leqslant n \leqslant N}} \pi_{1}^{\prime} f(m) \pi_{1}^{\prime} f(n)+ \\
+ & o_{f}\left(\left(\sum_{n} \frac{\pi_{2} f(n)}{f^{\prime}(n)}\right)\left((b-a) \sum_{m} f^{\prime}(m) \pi_{1}^{\prime} f(m)+\sum_{m} \pi_{2} f(m)\right)+\right. \\
& \left.+\left(\sum_{n} \pi_{1}^{\prime} f(n)\right)\left(\sum_{m} \frac{\pi_{2} f(m)}{f^{\prime}(m)}+(b-a)^{2} \sum_{m} \frac{f^{\prime \prime}(m)}{f^{\prime}(m)} \pi_{1}^{\prime} f(m)\right)\right) .
\end{aligned}
$$

LEMMA 2. Fïr $f$ aus $\mathscr{F}^{* *}$ und $0 \leqslant a<b \leqslant 1$ ist

$$
\int_{a}^{b} S_{M}^{\mathrm{nat}}(\mathscr{P} ; x) S_{N}^{\mathrm{mat}}(\mathscr{P} ; x) d x \leqslant(b-a) \Pi(M) \Pi(N)+O_{f}\left(R_{z}(a, b ; M, N)\right)
$$

mit

$$
\begin{gathered}
R_{2}(a, b ; M, N)=R_{31}(N)\left((b-a) R_{22}(M)+R_{23}(M)\right)+ \\
\quad+\Pi(N)\left(R_{21}(M)+(b-a)^{2} R_{24}(M)\right), \\
R_{21}(N)=\sum_{n=1}^{N} \frac{\pi_{2}(f(n))}{f^{\prime}(n)}, \\
R_{22}(N)=\sum_{n=1}^{N} f^{\prime}(n) \pi_{1}^{\prime}(f(n)), \\
R_{23}(N)=\sum_{n=1}^{N} \pi_{2}(f(n)), \\
R_{24}(N)=\sum_{n=1}^{N} \frac{f^{\prime \prime}(n)}{f^{\prime}(n)} \pi \pi_{1}^{\prime}(f(n)) .
\end{gathered}
$$

Für $M=N$ erweist sich die Abschätzung in Lemma 2 als unbradichbar, weil dann der $O$-Term den Hauptterm majorisiert. In dieser Situation behandeln wir das Integral $\int_{a}^{b} S_{N}^{\varphi}(\mathscr{P} ; x)^{2} d x$ wie folgt: Formel (5) 
wird eingesetzt in die rechte Seite der Gleichung

$$
\int_{a}^{b} S_{N}^{\varphi}(x)^{2} d x=\sum_{n=1}^{N} \frac{1}{\psi(n)^{2}} \int_{a}^{b} s_{n}(x)^{2} d x+2 \sum_{n=2}^{N} \sum_{m=1}^{n-1} \frac{1}{\psi(m) \psi(n)} \int_{a}^{b} s_{m}(x) s_{n}(x) d x .
$$

Als Hauptterm erscheint $(b-a) \Pi(N)^{2}$, und an $O$-Texmen treten auf

$$
\begin{aligned}
& T_{1}^{\prime}=(b-a) \sum_{n=1}^{N} \frac{\pi_{2} f(n)}{\psi(n) f^{\prime}(n)} \sum_{m=1}^{n} \frac{f^{\prime}(m) \pi_{1}^{\prime} f(m)}{\psi(m)} \\
& T_{3}^{\prime}=\sum_{n=1}^{N} \frac{\pi_{2}^{\prime} f(n)}{\psi(n)} \sum_{m=1}^{n} \frac{\pi_{2} f(m)}{\psi(m) f^{\prime}(m)} \\
& T_{4}^{\prime}=\sum_{n=1}^{N} \frac{\pi_{2} f(n)}{\psi(n) f^{\prime}(n)} \sum_{m=1}^{n} \frac{\pi_{2} f(m)}{\psi(m)} \\
& T_{6}^{\prime}=(b-a)^{2} \sum_{n=1}^{N} \frac{\pi_{1}^{\prime} f(n)}{\psi(n)} \sum_{m=1}^{n} \frac{f^{\prime \prime}(m) \pi_{1}^{\prime} f(m)}{f^{\prime}(m) \psi(m)}
\end{aligned}
$$

Damit ergibt sich als dritte Abschätzung

LeñuA 3. Fiü $f$ aus $\mathscr{F}^{* * *}$ und $0 \leqslant a<b \leqslant 1$ ist

$$
\int_{a}^{b} S_{N}^{\Psi}(\mathscr{P} ; x)^{2} d x \leqslant(b-a) \Pi(N)^{2}+O_{f}\left(R_{3}(a, b ; N)\right)
$$

mit

$$
\begin{gathered}
R_{3}(a, b ; N)=(b-a) R_{31}(N)+(b-a)^{2} R_{32}(N)+R_{33}(N) \\
R_{31}(N)=\sum_{n=1}^{N} \frac{\pi_{2}(f(n))}{\psi(n) f^{\prime}(n)} \sum_{m=1}^{n} \frac{f^{\prime}(m) \pi_{1}^{\prime}(f(m))}{\psi(m)} \\
R_{32}(N)=\sum_{n=1}^{N} \frac{\pi_{1}^{\prime}(f(n))}{\psi(n)} \sum_{m=1}^{n} \frac{f^{\prime \prime}(m) \pi_{1}^{\prime}(f(m))}{f^{\prime}(m) \psi(m)} \\
R_{33}(N)=\sum_{n=1}^{N}\left(\frac{\pi_{1}^{\prime} f(n)}{\psi(n)} \sum_{m=1}^{n} \frac{\pi_{2} f(m)}{\psi(m) f^{\prime}(m)}+\frac{\pi_{2} f(n)}{\psi(n) f^{\prime}(n)} \sum_{m=1}^{n} \frac{\pi_{2} f(m)}{\psi(m)}\right) .
\end{gathered}
$$

Mit der Hölderschen Ungleichung folgt aus den Lemmata 1 und 3 : Corollar. Mit $R_{i}(N)=R_{i}(0,1 ; N)(i=1,3)$ ist

$$
\int_{0}^{1}\left|\frac{S_{N}^{\varphi}(\mathscr{P} ; x)}{\Pi(N)}-1\right| d x=O_{f}\left(\Pi(N)^{-1}\left(I(N) R_{1}(N)+R_{3}(N)\right)^{1 / 2}\right) .
$$

Aus dem Corollax folgt

$$
\varliminf_{N \rightarrow \infty} \frac{S_{N}^{\varphi}(x)}{\Pi(N)}=1 \quad \text { für fast alle } n \text { ans } I^{1},
$$

soferm nur der $O$-Term des Corollars hinreichend gut gegen 0 konvergiert. Andererseits ist

$$
\sum_{n=1}^{N} \frac{\chi_{\mathscr{P}}(n)}{\psi_{2}(n)} \sim \sum_{n=1}^{N} \frac{\pi_{1}^{\prime}(n)}{\psi_{9}(n)}
$$

sobald die linke Seite in (7) gegen $\infty$ konvergiext, und das kann im Hinblick auf die Definition der Dichte

$$
D_{y_{2}}^{\psi_{1}}(\mathscr{P}, f)(x)=\lim _{N \rightarrow \infty} \frac{S_{N}^{w_{1}}(\mathscr{P} ; x)}{\sum_{1 \leqslant n \leqslant N} \frac{\chi_{\mathscr{P}}(n)}{\psi_{2}(n)}}
$$

vorausgesetzt werden. Aus (6), (7) und (8) läßt sieh erkennen: $\psi_{1}$ und $\psi_{2}$ sind so zu wählen - eventuell bis auf eine von 0 verschiedene nultiplikative Konstante, daß

$$
I I(N) \sim \sum_{n=1}^{N} \frac{\pi_{1}^{\prime}(n)}{\psi_{2}(n)}
$$

gilt. Die Definition $\Pi(N)=\sum_{n=1}^{N} \frac{\pi_{1}^{\prime} f(n)}{\psi_{1}(n)}$ zeigt nun, daB bei vorgegebenem $\psi_{1}$

$$
\psi_{2}(n) \sim \frac{\pi_{1}^{\prime}(n)}{\pi_{1}^{\prime} f(n)} \psi_{1}(n)
$$

die nächstliegende Wahl für $\psi_{2}$ darstellt.

3. Anwendungen. Als erstes wollen wir untersuchen, wie die Pseudoprimzahlen $P^{*}=\left\{\left[1 \mathrm{i}^{-1}(n)\right] ; n \geqslant 2\right\}$ in Folgen der Form $[f(n+x)]_{n \in \mathbf{N}}$
verteilt sind. Für $P^{*}$ ist

$$
\pi_{P^{*}}(y)=\operatorname{li}(y)+O(1)
$$

und die Funktionen $\pi_{1}(y)=\operatorname{li}(y), \quad \pi_{1}^{\prime}(y)=\log ^{-1} y, \pi_{2}(y)=1$ erfüllen die zu Beginn ron Teil 2 gestellten Bedingungen.

Sei zunächst $f$ aus $\mathscr{F}_{\text {pol }}^{*}$ rom Grad $k>2$. Wir wichten mit $\psi(y)=y$. Lemma 1 liefert

$$
\Pi(N)=\sum_{n=1}^{N} \frac{1}{n \log f(n)} \sim \frac{1}{k} \log \log N
$$


$R_{11}(N)=O_{f}(1)$ und $R_{12}(N)=O_{f}(1)$. Also wird

$$
\int_{0}^{1} S_{N}^{\text {har }}\left(P^{*} ; x\right) d x=\Pi(N)+O_{f}(1) \text {. }
$$

Die $O$-Terme $R_{31}(N), R_{32}(N)$ und $R_{33}(N)$ in Lemma 3 sind beschränkt durch $O_{f}(\log \log N)$, und das führt auf

$$
\int_{0}^{1} S_{N}^{\mathrm{har}}\left(P^{*} ; x\right)^{2} d x \leqslant \Pi(N)^{2}+O_{f}(\log \log N)
$$

Zusammen folgt mit dem Corollar zu Lemma 3

$$
\int_{0}^{1}\left|\frac{S_{N}^{\operatorname{har}}\left(P^{*} ; x\right)}{\Pi(N)}-1\right| d x=O_{f}\left\{(\log \log N)^{-1 / 2}\right\} .
$$

Mit $g(N)=3^{3^{N^{4}}}$ ist $\sum_{Y=1}^{\infty}(\log \log g(N))^{-1 / 2}<\infty$, und deshalb ergibt sich aus (3) und (2)

$$
\lim _{N \rightarrow \infty} \frac{S_{g(N)}^{\mathrm{har}}}{\log \log g(N)}=\frac{1}{\mu} \text {. }
$$

Der nun folgende Konvergenzschluß wird verschiedentlich anftreten; deshalb widmen wir ihm einen

HHFSS.ATz 1. Ist $\left(a_{n}\right)_{n \in N}$ eine beschränkte Folge nichtnegativer reeller Zahlen und sind $g$ und $h$ monoton gegen $\infty$ wachsende Frunktionen anf $\boldsymbol{N}$ mit $\lim _{N \rightarrow \infty} \frac{h g(N+1)}{h g(N)}=1$, so konvergiert die Folge der gewichteten Mittel

$$
A_{N}=\frac{1}{h(N)} \sum_{n=1}^{N} a_{n}
$$

schon dann, wenn 'die Teilfolge $\left(A_{g(N)}\right)_{N \in \mathbf{N}}$ konvergiert.

Mit $a_{n}=\frac{1}{n} \chi P^{*}([f(n+x)])$ und $h(N)=\log \log N$ zeigt Hilfssatz 1 bei Anwendung auf (4)

$$
\lim _{N \rightarrow \infty} \frac{S_{N}^{\mathrm{har}}}{\log \log N}=\frac{1}{\hbar} .
$$

Schließlich ergibt sich aus (1)

$$
\sum_{1 \leqslant n \leqslant N} \frac{1}{n} x_{P^{*}}(n)=\log \log N+O(1)
$$

and damit wird $D_{\mathrm{har}}^{\mathrm{bar}}\left(P^{*}, f\right) \underset{\mu}{\bar{\mu}} \frac{1}{k}$.
Die Funktionen $f$ aus $\mathscr{F}_{\text {exp }}^{*}$ wichten wir mit $\psi(y)=1$. Für den Hauptterm in Lemma 1 ergibt sich dann

$$
\Pi(N)=\sum_{n=1}^{N} \frac{1}{\log f(\eta n)} \sim \frac{\log N}{k}
$$

und $R_{11}(N)=O_{f}(\Pi(N)), \quad R_{19}(N)=O_{f}(1)$. Wird nun $I^{1}$ in $[\sqrt{\Pi(N)}]$ Intervalle gleicher Länge zerlegt, wird auf diese Intervalle Lemma 1 angewandt und werden dann die so erhaltenen Gleichungen addiert,
folgt

$$
\int_{0}^{1} S_{N}^{\text {nat }}\left(P^{*} ; x\right) d x=\Pi(N)+O_{f}(\sqrt{\Pi(N)}) .
$$

Für die $O$-Terme in Lemma 3 gelten die Abschätzungen $R_{31}(N)=O_{f}(\log N)$, $R_{32}(N)=O_{f}\left(\log ^{2} N\right)$ and $R_{33}(N)=O_{f}(\log N)$. Zexlegen wir $I^{1}$ wie eben, wenden Lemma 3 an und addieren, so erhalten wir

$$
\int_{0}^{1} S_{N}^{\text {nath }}\left(P^{*} ; x\right)^{2} d x \leqslant \Pi(N)^{2}+O_{f}\left(\log ^{3 / 2} N\right),
$$

und das Corollar zu Lemma 3 zeigt

$$
\int_{0}^{1}\left|\frac{S_{N}^{\mathrm{nat}}\left(P^{*} ; x\right)}{\Pi(\bar{N})}-1\right| d x=O_{f}\left(\log ^{-1 / 4} N\right) .
$$

Mit $g(N)=3^{N^{8}}$ ist unter Beachtung von (5) also

$$
\lim _{N \rightarrow \infty} \frac{S_{\mathscr{R}(N)}^{\mathrm{mat}}}{\log g(N)}=\frac{1}{k}
$$

Hilfssatz 1 mit $h(N)=\log N$ liefert daraus

$$
\lim _{N \rightarrow \infty} \frac{S_{N}^{\text {nat }}}{\log N}=\frac{1}{k}
$$

und so folgt in Verbindong mit

$$
\sum_{1 \leqslant n \leqslant N} \frac{1}{\operatorname{li}(n)} \chi_{P^{*}}(n) \sim \log N
$$

schlieblich $D_{\mathrm{li}}^{\text {nat }}\left(P^{*}, f\right)=1 / k$. Damit ist bewiesen:

SATZ 4. Fiür die Diohte der Pseudoprimzahlen $P^{*}$ in Folgen der Form $[f(n+\infty)]_{n \in \mathbf{N}}$ gilt:

$$
\begin{array}{ll}
D_{\mathrm{har}}^{\mathrm{har}}\left(P^{*}, f\right)=\frac{1}{k} & \text { für } f \text { aus } \mathscr{F}_{\text {pol }} \text { vom Grad } k>2, \\
D_{\mathrm{li}}^{\mathrm{nat}}\left(P^{*}, f\right)=\frac{1}{k} \quad \text { für } f \text { aus } \mathscr{F}_{\text {exp }} \text { vom Grad } k .
\end{array}
$$


Bemerkungenl. (1) Daß für $f$ ans $\mathscr{F}_{\text {pol }}$ sogar $D_{\text {nat }}^{\text {nat }}\left(P^{*}, f\right)=\frac{1}{\mu}$ gilt, läßt sich nicht in ähnlicher Weise zeigen. Bei Wichtung mit $\psi(y)=1$ wird nämlich der $O$-Term $R_{31}(N)$ in Lemma 3 um den Falktor $\log N$ größer als der Flauptterm $\Pi(N)^{2}$.

(2) Nit Abelscher partieller Summation folgt aus dem zweiten Ergeb nis des Satzes auch $D_{\mathrm{har}}^{\log }\left(P^{*}, f\right)=\frac{1}{\mu}$.

Nachdem Satz 4 gezeigt hat, welche Resultate für die Verteilung der Primzahlen in den Folgen $[f(n+x)]_{n \in \mathbb{N}}$ zu erwaxten sind, soll num untersucht werden, was davon in ähnlicher Weise sich zeigen läßt. Dazu werden die in der Einleitung angeführten Primzahlsätze (PZS 1) und (PZS 2) herangezogen. Lemma 3 ist weder für Funktionen ans $\mathscr{F}_{\text {pol }}^{*}$ noch fürr solche aus $\mathscr{F}_{\exp }^{*}$ anwendbar; ganz unabhängig ron der WahI möglicher Wichtungsfunktionen $\psi$ übertrifft der 0 -Term $R_{31}(N)$ den Hanptterm $\Pi(N)^{2}$ jeweils bei weitem.

Für $f$ aus $\mathscr{F}_{\text {exp }}^{*}$ und Wichtung $\psi(y)=1$ árgibt sich bei Benutzung. von (PZS 1) in Lemma 1

$$
\begin{gathered}
\Pi(N)=\sum_{n=1}^{N} \log ^{-1} f(n)=O_{f}(\log N), \\
R_{11}(N)=O_{f}(I(N)) \quad \text { and } \quad R_{12}(N)=O_{f}(1) .
\end{gathered}
$$

Jedes vorgegebene Intervall $\left[a, b\left[\right.\right.$ in $I^{1}$ zerlegen wir in $[\sqrt{\Pi(\mathcal{N})}]$ Intervalle gleicher Länge, wenden auf diese Lemma 1 an, addieren und erhalten. so

$$
\int_{a}^{b} \frac{S_{N}^{\mathrm{nat}}(P ; x)}{\Pi(N)} d x=b-a+O_{f}\left(\log ^{-1 / 2} N\right)
$$

Für Funktionen $f$ aus $\mathscr{F}_{\text {pol }}^{*}$ liefert Lemma 1 nichts, wenn nur (PZS 1 ) zugelassen wird, da dann $R_{12}(N)$ den Hauptterm $\Pi(N)$ übertrifft. Anwendung ron (PZS 2) ergibt aber bei Wichtung $\psi(y)=1$

$$
\begin{gathered}
\Pi(N)=\sum_{n=1}^{N} \log ^{-1} f(n)=O_{f}\left(\frac{N}{\log N}\right) \\
R_{11}(N)=O_{f}(\log \log N) \quad \text { und } \quad R_{12}(N)=O_{f}\left(N^{2-k / 2} \log N\right) \quad \text { für } k>2
\end{gathered}
$$

und es wird

$$
\int_{a}^{b} \frac{S_{N}^{\text {mat }}(P ; x)}{\Pi(N)} d x=b-a+O_{f}\left(N^{1-1 / 2} \log ^{2} N\right)
$$

SATZ 5. (i) Für Funktionen $f$ aus $\widetilde{F}_{\exp }$ nom Grad $k$ ist

$$
\liminf _{N \rightarrow \infty} D_{\mathrm{li}}^{\text {nat }}(P, f ; N) \leqslant \frac{1}{k} .
$$

(ii) Seif aus $\mathscr{F}_{\text {pol }}$ vom Grad $\gamma_{>}>2$. Ist der Primzahlsatz $\pi_{P}(y)=\operatorname{li} y+$ $+O(\sqrt{y} \log y)$ richtig, so folgt

$$
\liminf _{N \rightarrow \infty} D_{\text {nat }}^{\text {nat }}(P, f ; N) \leqslant \frac{1}{k}
$$

Beweis. Für die Funktionen aus $\mathscr{F}_{\text {pol }}^{*}$ rom Grad $k>2$ und die aus $\mathscr{F}_{\exp }^{*}$ gilt gemäß (6) und $(7)$

(8) $\quad \int_{a}^{b} \frac{S_{N}^{\mathrm{nat}}(x)}{\Pi(N)} d x=b-a+0_{f}(R(N))$ mit $\lim _{N \rightarrow \infty} R(N)=0$.

Hieraus folgt für alle offenen Mengen $A$ in $[0,1]$

$$
\liminf _{N \rightarrow \infty} \int_{A} \frac{S_{N}^{\text {nat }}(x)}{I(N)} d x \geqslant \mu(A)
$$

denn $A$ ist abzählbare Vereinigung paarweise disjunkter offener Intervalle $A_{i}, i \in N$, deshalb folgt aus (8) mit einer positiven Konstante $C_{f}$ für jedes $n k$

$$
\int_{i} \frac{S_{N}^{\mathrm{nat}}(x)}{\Pi(N)} d x \geqslant \int_{i \leqslant m} \frac{S_{N}^{\mathrm{mat}}(x)}{\Pi(N)} d x \geqslant \mu\left(\bigcup_{i \leqslant n} A_{i}\right)-\sigma_{f} m R(N),
$$

und daraus ergibt sich für $m=\left[R(N)^{-1 / 2}\right]$ und $N \rightarrow \infty$ Formel (9). Für abgeschlossenes $B$ in $[0,1]$ folgt aus $(9)$

$$
\limsup _{N \rightarrow \infty} \int_{B} \frac{S_{N}^{\text {nat }}(x)}{\Pi(N)} d x \leqslant \mu(B)
$$

Eür $\varepsilon>0$ und natürliche Zahlen $M I$ sind die Mengen

$$
A_{\varepsilon_{3} I I}=\left\{x \in I^{1} ; \frac{S_{N}^{\mathrm{nat}}(x)}{\Pi(N)}<1+\varepsilon \text { für ein } N \geqslant M\right\}
$$

als abzählbare Vereinigungen von Intervallen $\mu$-offen; die Komplemente $B_{\varepsilon, \Delta I}=I^{1}-A_{\varepsilon, \text { XI }}$ sind also $\mu$-abgeschlosser, und auf sie läßt sich (10) anwenden:

$$
(1+\varepsilon) \mu\left(B_{\varepsilon, M}\right) \leqslant \limsup _{N \rightarrow \infty} \int_{B_{\varepsilon, M M}} \frac{S_{N^{*}}^{\text {nat }}(x)}{\Pi(N)} d x \leqslant \mu\left(B_{\varepsilon, M}\right) .
$$


Also ist $\mu\left(B_{\varepsilon, M}\right)=0$. Dann ist aber $\mu\left(A_{\varepsilon, M}\right)=1$ für jedes positive $\varepsilon$ und alle $M$, und daraus folgt $\liminf _{N \rightarrow \infty} \frac{S_{N}^{\text {nat }}}{\Pi(N)} \leqslant 1$. Das zusammen mit

$$
\lim _{N \rightarrow \infty} \frac{\Pi(N)}{\sum_{1 \leqslant n \leqslant N} \chi_{P}(n)}=\lim _{N \rightarrow \infty} \frac{\sum_{1 \leqslant n \leqslant N} \log ^{-1} f(n)}{\operatorname{li} N}=\frac{1}{k} \text { für } f \text { aus } \mathscr{F}_{\text {pol }}^{*}
$$

and

$$
\lim _{N \rightarrow \infty} \frac{\Pi(N)}{\sum_{1 \leqslant n \leqslant N} \frac{1}{\operatorname{li} n} \chi_{P}(n)}=\lim _{N \rightarrow \infty} \frac{\sum_{1 \leqslant n \leqslant N} \log ^{-1} f(n)}{\log N}=\frac{1}{k} \quad \text { für } f \text { aus } \mathscr{F}_{\operatorname{axp}}^{*}
$$

ergibt die beiden Behauptungen des Satzes.

Als drittes Beispiel untersuchen wir Mengen $\mathscr{P}$ mit positiver natürlicher Dichte in $\boldsymbol{N}$ und verhältnismäßig schwach wachsender Fehlerfunktion $\pi_{2}(y)$ : funktion

SATZ 6. Sei PP eine Teilmenge der natillichen Zahlen mit der Anzahl-

$$
\pi_{\mathscr{P}}(y)=\gamma y+O\left(\log ^{\delta} y\right), \quad \gamma>0,0 \leqslant \delta<1 .
$$

(i) Für $f$ aus $\mathscr{F}_{\text {pol }}$ vom Grad $k>2$ ist $D_{\text {har }}^{\text {har }}(\mathscr{P}, f)=1$.

(ii) Fïur $f$ ans $\mathscr{F}_{\exp }$ ist $D_{\text {nat }}^{\text {nat }}(\mathscr{P}, f)=1$.

Beweis. $\pi_{1}(y)=\gamma y$ und $\pi_{2}(y)=\log ^{d} y$ erfüllen die zu Anfang von Teil 2 gestellten Bedingungen. Ferner darf angenommen werden, daß $f$ der Forderung (F1) genügt.

(i) Bei Wichtung $\psi(y)=y$ wird $\Pi(N)=\sum_{n=1}^{N} \frac{\gamma}{n}=\gamma \log N+O(1)$, und für die $O$-Terme der Lemmata 1 and 3 erhalten wir $R_{1}(0,1 ; N)$ $=O_{f}(\mathcal{1})$ und $R_{3}(0,1 ; N)=O_{f}\left(\log ^{1+\delta} N\right)$. Mit dem Oorollar zu Lemma 3 folgt daraus

$$
\int_{0}^{1}\left|\frac{S_{N}^{\operatorname{har}}(x)}{\Pi(N)}-1\right| d x=o_{f}\left(\log ^{-(1-\delta) / 2} N\right)
$$

Für natürliches $t>2 /(1-\delta)$ and $g(N)=3^{N^{t}}$ ist $\sum_{N=1}^{\infty} \log ^{-(1-\delta) / 2} g(N)<\infty$, und deshalb. folgt aus (11) zunächst $\lim _{N \rightarrow \infty} \frac{S_{g(N)}^{\text {har }}}{\Pi(g(N))}=1$ und dann mit
Hilfssatz 1 auch $\lim _{N \rightarrow \infty} \frac{S_{N}^{\text {har }}}{\Pi(N)}=1$. Im Hinblick auf

$$
\sum_{1 \leqslant n \leqslant N} \frac{x_{\mathcal{P}}(n)}{n} \sim \Pi(N)
$$

ist das die Behauptung.

(ii) Wichtung mit $\psi(y)=1$ führt auf $\Pi(N)=\gamma N$. Die 0 -Terme in Lemma $I$ sind $R_{11}(N)=O_{f}(N)$ und $R_{12}(N)=O_{f}(1)$. Wie schon in den ersten Beispielen zerlegen wir $I^{1}$ wieder in $[\sqrt{\Pi(N)}]$ gleichlange Intervalle, wenden auf diese Lemma 1 an, addieren and exhalten

$$
\int_{0}^{1} S_{N}^{\text {mat }}(x) d x=\gamma N+O_{f}(\sqrt{N})
$$

Dasselbe Vorgehen führt bei Lemma 3 wegen $R_{31}(N)=O_{f}\left(N^{\mathrm{I}+\delta}\right), R_{32}(N)$ $=O_{f}\left(N^{2}\right)$ and $R_{33}(N)=O_{f}(N)$ auf

$$
\int_{0}^{1} S_{N}^{\text {mat }}(x)^{2} d x \leqslant \gamma^{2} N^{2}+O_{f}\left(N^{1+\delta^{\prime}}\right), \quad \delta^{\prime}=\max \left\{\delta, \frac{1}{2}\right\} .
$$

Insgesamt ergilut sich mit dem Corollar zu Lemma 3

$$
\int_{0}^{1}\left|\frac{S_{N}^{\text {nat }}(x)}{\gamma N}-1\right| d x=O_{f}\left(N^{-\left(1-\delta^{\prime}\right) / 2}\right) .
$$

Daraus folgt für hinreichend großes ganzes $t$ und $g(N)=N^{t}$

$$
\lim _{N \rightarrow \infty} \frac{S_{g(N)}^{\text {nat }}}{\gamma g(N)}=1
$$

und Hilfssatz 1, zusammen mit $\sum_{n=1}^{N} \chi_{g}(n) \sim \gamma N$, liefert Aussage (ii).

Bemerkungen. (1) Für jede natürliche Zahl $a$ erfüllt $\mathscr{P}=d N$ die Voraussetzungen von Satz 6. So ergeben sich Aussagen über die Teilbarkeit der Folgenelemente $[f(n+x)]$ durch $d$.

(2) Das Ergebnis ron Satz 6(i) läBt sich mit den im Beweis angewandten Schlüssen nicht verbessem zu einer Konvergenzaussage für die natürliche Dichte. Das gelingt selbst dann nicht, wenn $\mathscr{P}$ der sehärferen Bedingung $\pi_{\mathscr{F}}(y)=\gamma y+O(1)$ genügt. In jedem Fall nämlich erreicht für $\psi(y)=1$ der 0 -Term $R_{31}(N)$ in Lemma 3 dieselbe Größenordnung wie der Hauptterm. Immerhin aber besagt (11) für $\mathscr{P}=d \boldsymbol{N}$

$$
\int_{0}^{1}\left|S_{N}^{\mathrm{har}}(d \boldsymbol{N} ; x)-\frac{\log N}{d}\right| d x=O_{f}(\sqrt{\log N}) .
$$


Ond dabei kann die Konstante zu. $O_{f}$ so gewählt werden, daß (12) mit $f$ zugleich auch für alle Funktionen $f_{n}(y)=f(n+y), n \in N$, richtig ist. Wenden wir (12) an auf $f_{n}$ anstelle von $f$ und auf $N-n$ statt $N$, dividieren die so erhaltene Gleichung durch $\log (n+2)$ und summieren über $n$ von 0 bis $N-1$, so folgt für $f$

$$
\int_{0}^{1}\left|S_{N}^{p}(d \mathbf{N} ; x)-\frac{1}{d} \sum_{n=0}^{N-1} \frac{\log (N-n)}{\log (n+2)}\right| d x=O_{f}\left(\sum_{n=0}^{N-1} \frac{\sqrt{\log (N-n)}}{\log (n+2)}\right)
$$

mit

$$
\psi(n)=\left(\sum_{m=1}^{n} \frac{1}{m \log (n-m+2)}\right)^{-1}
$$

Zusammen mit den Gleichungen

$$
\begin{gathered}
\sum_{n=1}^{N} \frac{1}{n \log (N+2-n)}=1+O\left(\log ^{-1} N\right) \\
\sum_{n=0}^{N-1} \frac{\log (N-n)}{\log (n+2)}=N+O\left(N \log ^{-1} N\right) \\
\sum_{n=0}^{N-3} \frac{\sqrt{\log (N-n)}}{\log (n+2)}=\frac{N}{\sqrt{\log N}}+O\left(N \log ^{-3 / 2} N\right)
\end{gathered}
$$

ergibt sich dann für $f$

$$
\int_{0}^{1}\left|S_{N}^{\mathrm{nat}}(d N ; x)-\frac{N}{d}\right| d x=o_{f}\left(\frac{N}{\sqrt{\log N}}\right) .
$$

In dieser Situation ist aber Hilffssatz 1 nicht anwendbar; es folgt lediglich

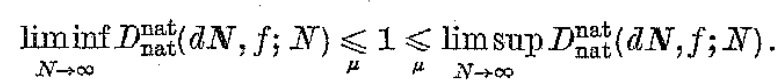

(3) Gleichwohl wird sich in Teil 5 herausstellen, daß $D_{\text {nat }}^{\text {nat }}(d N, f) \underset{\mu}{\rightleftharpoons} 1$ für Funktionen aus $\mathscr{F}_{\text {pol }}$ vom Grad $k \geqslant 2$ richtig ist. Das Entscheidende im Hinblick auf die Anwendbarkeit der Teilbarkeitsaussagen in den Folgen $[f(n+x)]_{n \in \mathbf{N}}$ bei dem Selbergschen Sieb in Teil 6 ist aber, daß sich der 0 -Term von Formel (13) wesentlich verkleinern läßt, wie ein Vergleich mit Satz 9 zeigt. Entscheidend ist das deshalb, weil das Selberg-Sieb weder mit dem 0 -Term aus (13) noch mit der Wichtung $\psi(y)=y$ aus Satz 6(i) das im Beweis von Satz 11 benötigte Ergebnis (i) in Hilfssatz 4 liefert. Daß der 0 -Term in Satz 9 sehr viel besser ist als der in Formel (13), hat folgenden Grund: Zum Beweis der Ungleichung in Lemma 3 wurden für festes $n$ alle Integrale

$$
\int_{a}^{b} s_{m}(x) s_{n}(x) d x \quad(1 \leqslant m \leqslant n)
$$

unabhängig voneinander nach oben abgeschätzt. Dazu war die jeweils ungünstigste Konstellation von $s_{m}$ in Bezug auf $s_{n z}$ zu berücksichtigen. Durchläuft aber $m$ die Zahlen 1 bis $n$, so treten angünstige Konstellationen gar nicht so häufig aut und werden überdies zumindest teilweise kompensiert durch besonders günstige. In Teil 4 wird ein - dort als solcher noch nicht interpretierter - Mittelwert über die Lage der $s_{m n}$ bezüglich $s_{n}$ errechnet, und in Teil 5 wird dieser dann benutzt, um (13) zu der Aussage von Satz 9 zu verbessern.

\section{Maximalabstände in endlichen arithmetischen Folgen modnlo 1 .} Die Theorie der diophantischen Approximationen behandelt für irrationale Zahlen $x$ Eigenschaften der Folgen $(n x)_{n=v_{0}}$; vergl. etwa Kolssma [16], Kap. IX. Bekanntlich sind diese Folgen gleichverteilt modulo 1. Eingehendere Untersuchungen benutzen den Begriff der Diskrepanz

$$
D_{N}(x)=\sup _{0 \leqslant \alpha<\beta \leqslant 1} \mid \frac{1}{N} \mathrm{~A}\left(\left[\alpha, \beta\left[;\{n x\}_{0 \leqslant n<N}\right)-(\beta-\alpha) \mid .\right.\right.
$$

Khintchine [15] hat gezeigt, daß für jedes positive $\varepsilon$ und fast jedes reelle $x$ $D_{N}(x)=o\left(N^{-1} \log ^{1+5} N\right)$ gilt. Für $\dot{\varepsilon}=0$ wird diese Aussage falsch. Für Trrationalzahlen $x$ mit beschränkten Teilnennein der regelmäßigen Kettenbruchentwicklung hat Ostrowski in [24] sogar $D_{N}(x)=O_{x}\left(N^{-1} \log N\right)$ bewiesen.

Uns interessiert hier für beliebiges $x \geqslant 0$ und $y \geqslant 1$ eine der Diskrepanz ähnliche Funktion, nämlich der maximale unter den Abständen je zweier benachbarter Punkte der endlichen Folge $(n x)_{0 \leqslant n<\{y\}}$ modulo 1 , also

$$
\mathfrak{D}_{y}(x)=\sup _{0 \leqslant \alpha<\beta \leqslant 1}\left\{\beta-\alpha ; \mathrm{A}\left(\left[\alpha, \beta\left[;\{n x\}_{0 \leqslant n<[y]}\right)=0\right\} .\right.\right.
$$

Offensichtlich ist immer $\grave{D}_{y}(x) \leqslant D_{[y]}(x)$. Zunächst wird eine Rekursionsformel zur Berechnung von $\mathrm{D}_{y}$ bewiesen; Satz 7 beschreibt dann den Funktionsverlauf von $\mathfrak{b}_{y}$. Daraus wird eine in Teil $\breve{b}$ benötigte Abschätzung $\operatorname{von} \int_{0}^{1} \mathfrak{D}_{y}(x) d x$ hergeleitet.

Für positives $y$ bezeichne $\mathfrak{F}_{y}$ die Farey-Reihe der Ordnung $y$, also die der Größe nach geordncten Brüche mit nichtnegativen, je teilerfremden, durch $y$ beschränkten Zählern und Nennern im Intervall $[0,1]$. Sind $a / b$ wnd $c / d$ benachbart in der Farey-Reihe $\mathfrak{F}_{y}$ und gilt $a / b<c / d$, so schreiben wir kurz $a / b \underset{y}{<} c / d$. Zn $a / b<_{y} c / d$ heiBt $(a+c) /(b+d)$ der Mediant der 
benachbarten Brüche. Bei Betrachtung modulo 1 zerfällt der Kreis $[0,1[$ mit Umfang 1 in Bögen, deren Anfangs- und Endpunkte je benachbarte Medianten sind, und jeder diesex Farey-Bögen enthält genau einen Bruch $a / b$ der Farey-Reihe. Dieser Bruch $a / b$ zerlegt seinen Farey-Bogen $F_{a / b}$ in zwei Teile $F_{a / b}^{1}$ und $F_{a j b}^{2}$. Die so hergestellte Zerlegung des Kreises heibt Farey-Zerlegung der Ordnung $y$.

HEFSSATZ 2. (i) (Farey-Canchy) Aus $a / b<c / d$ folgt $b c-a d=1$.

(ii) In der Farey-Zerlegung der Ordnung $y \geqslant 2$ gitt für die Lü̈nge jedes der beiden Teile $F_{a / b}^{1}(i=1,2)$ des Farey-Bogens $F_{a / b}$

$$
\frac{1}{b(2[y]-1)} \leqslant \mu\left(F_{a / b}^{i}\right) \leqslant \frac{1}{b([y]+1)} .
$$

Beweis. Hardy-Wright [12], Theoreme 28 und 35 .

Reduktions-Lencha. Für $x \in] 0,1]$ und $y \geqslant 1$ ist

$$
\mathfrak{D}_{y}(x)=\left\{\begin{array}{lll}
\max \{x, 1-[y-1] x\} & \text { für } & {[y] \leqslant 1+\frac{1}{x},} \\
x \cdot \mathfrak{D}_{x\{[y]-[1 / x])+1}(\{1 / x\}) & \text { für } & {[y]>1+\frac{1}{x} .}
\end{array}\right.
$$

Beweis. Für Intervalle $I$ endlicher Länge in $\boldsymbol{R}$ und endliche Mengen $M \subset I$ bezeichne $\max (I ; M)$ das Maximum der Abstände je zweier der Größe nach benachbarter Zahlen der Menge $M \cup \operatorname{Rand}(I)$.

Im Fall $[y] \leqslant 1+\frac{1}{x}$ ist $\mathfrak{b}_{y}(x)$ das Maximum der Differenzen aufeinanderfolgender Zahlen der Folge $0, x, 2 x, \ldots,[y-1] x, 1$; und das ist $\max \{x, 1-[y-1] x\}$.

Sei nun $[y]>1+\frac{1}{x}$. Wir können $\left.x \in\right] 0,1[$ annehmen, denn für $x=1$ ist die Formel offensichtlich richtig. Zu jedem $j$ aus $N_{0}$ existiert genau ein $r_{j}$ aus $\boldsymbol{N}_{0}$ mit

$$
\left(r_{j}-1\right) x<j \leqslant r_{j} x
$$

Wegen $x<1$ ist $r_{1} \geqslant 2$; außerdem gilt stets $\left\{r_{j} x\right\}=r_{j} x-j$.

Die Zahlen der Folge $\left(\{n x\}_{0}\right)_{0 n<[y]}$ verteilen sich auf die Intervalle $I_{m}=\left[m x,(m+1) x\left[, \quad 0 \leqslant m \leqslant r_{1}-2\right.\right.$, und $I_{-1}=[1-x, 1[$ wie folgt: In $I_{m}$ liegen genau die Zahlen $\left(\left.\left\{\left(m+r_{j}\right) x\right\}\right|_{-1, m \leqslant j \leqslant k(m)}\right.$, und dabei ist $k(m)=[y] x-(m+1) x$.

Denn für $m \geqslant 0$ folgt aus (1) $j \leqslant r_{j} x<j+\infty$, also $m x \leqslant\left(m+r_{j}\right) x-j$ $<(m+1) x$. Ist umgekehrt $\{n x\} \in I_{m}$, also $m a \leqslant\{n x\}<(m+1) x$, so folgen bei Addition von $[n x]-m x$ die Ungleichungen $[n x] \leqslant(n-m) x$ $<[n x]+x$, also $(n-m-1) x<[n x] \leqslant(n-m) x$, und (1) zeigt $r_{[n x]}=n-m$. Für $m=-1$ folgt aus $(1) j-x \leqslant\left(r_{j}-1\right) x<j$, also $1-x \leqslant\left\{\left(r_{j}-1\right) x\right\}<1$.
Ist umgekehrt $\{n x\} \in I_{-1}$, also $1-x \leqslant\{n s\}<1$, so folgt $[n x]+1-x$ $\leqslant n x<[n x]+1$ und daraus $n x<[n x]+1 \leqslant(n+1) x$, und (1) zeigt $r_{[n x]+1}=n+1$. SchlieBlich ist das jeweils größte $j$ bestimmt durch die Bedingung $m+r_{j} \leqslant[y-1] \leqslant m+r_{j+1}-1$, und daraus ergibt sich bei Multiplikation mit $x$ und unter Beachtung von (1) $j \leqslant[y-1] x-m x<j \div 1$.

Weil die Intervalle $I_{m}$ für $-1 \leqslant m \leqslant r_{1}-2$ das Intervall $[0,1[$ überdecken und. weil die Randpunkte der $I_{m}$ mit $m \neq-1$ Zahlen der Folge $\left(\{n x\}_{0 \leqslant n<\{y]}\right.$ sind, nicht aber notwendig die linke Intervallgrenze $1-\infty$ von $I_{-1}$, tritt mindestens eine der folgenden drei Möglichkeiten ein:

(i) $\triangleright_{y}(x)=\max _{0 \leqslant m \leqslant r_{1}-2}\left\{\max \left(I_{m} ;\left(\left\{\left(m+r_{j}\right) x\right\}\right\}_{0 \leqslant j \leqslant k(m)}\right)\right\}$,

(ii) $D_{y}(x)=\max \left(I_{-1} ;\left(\left\{\left(r_{j}-1\right) x\right\}\right)_{1 \leqslant j \leqslant k(-1)}\right)$,

(iii) $\mathfrak{D}_{y}(x)=\left\{n_{2} x\right\}-\left\{n_{1} x\right\}$ für zwei der Größe nach benachbarte Elemente $\left\{n_{1} x\right\}, \quad\left\{n_{2} x\right\}$ der Folge $\left(\{n x x)_{0 \leqslant n<[y]}\right.$ mit $\left\{n_{1} x\right\}<1-x \leqslant\left\{n_{2} x\right\}$.

Trifft (iii) zu, so folgt mit $\left(r_{1}-2\right) x<1-x \leqslant\left(r_{1}-1\right) x$ sofort $\left(r_{1}-2\right) x$ $\leqslant\left\{n_{1} x\right\}<\left\{n_{2} x\right\} \leqslant\left(r_{1}-1\right) x$, und dann ist auch (i) richtig. Ebenso ist (i) richtig; wenn (ii) gilt. Denn aus (1) folgt $j-1<\left(r_{j}-1\right) x<j$; die Abstände der Zahlen aus $I_{-1}$ zur rechten Intervallgrenze 1 von $I_{-1}$ sind demnach

$$
1-\left\{\left(r_{j}-1\right) x\right\}=j-\left(r_{j}-1\right) x=x-\left(r_{j} x-j\right)=x-\left\{r_{j} x\right\},
$$

und das sind wegen $k(0) \leqslant k(-1)$ für $1 \leqslant j \leqslant k(0)$ die Abstände der Zahlen $\left(\left\{r_{j} x\right\}\right)_{1 \leqslant j \leqslant k(0)}$ aus $I_{0}$ von $x$. (i) ist demnach richtig. Weil nun $\left.\max \left(I_{m} ;\left\{\left(m+r_{j}\right) x\right\}\right\}_{0 \leqslant i \leqslant k(m)}\right)$ invariant bleibt gegenüber gleichzeitiger Translation des Intervalls $I_{m}$ und der relevanten Punkte, und weil $k$ a.ls Funktion von $m$ monoton fällt, folgt ans (i) mit $k^{*}=k\left(r_{1}-2\right)$

$$
\begin{aligned}
\mathfrak{b}_{y}(x) & =\max \left(\left[0, x\left[;\left(\left\{r_{j} x\right\}\right)_{0 \leqslant j \leqslant k^{*}}\right)\right.\right. \\
& =\max \left(\left[0, x\left[;\left(r_{j} x-j\right)_{0 \leqslant j \leqslant k^{*}}\right)\right.\right. \\
& =x \max \left(\left[0,1\left[;\left(r_{j}-j \mid x\right)_{0 \leqslant j \leqslant k^{*}}\right) .\right.\right.
\end{aligned}
$$

Da $\mathfrak{b}_{y}(x)$ seinen Wert nicht ändert bei Spiegelung des Intervalls und der Punkte rechts in (2) am Punkt 1/2, folgt weiter

$$
\begin{aligned}
& \left.\left.\grave{D}_{y}(x)=x \max (] 0,1\right] ;\left(1-r_{j}+j|x\rangle_{0 \leqslant j \leqslant k^{*}}\right)=x \max (10,1] ;(\{j \mid x\})_{0 \leqslant j \leqslant k^{*}}\right) \\
& =x \mathrm{D}_{k^{*}+1}(\{1 / \infty\}) \text {. }
\end{aligned}
$$

Gemäß (1) ist $r_{1}-1<1 / x \leqslant r_{1}$. Für $1 / x<r_{1}$ folgt daraus [1/x] $=r_{1}-1$ und wir haben $k^{*}+1=[y] x-\left(r_{1}-1\right) x+1=x([y]-[1 / x])+1$. Für $1 / x=r_{1}$ ist einerseits $\mathrm{D}_{y}(x)=x$ und andererseits

$$
\mathfrak{D}_{x([y]-[1 / x])+1}(\{1 / x\})=1,
$$

weil 1/x ganzzahlig ist. Damit ist die Rekursionsformel bewiesen. 
SATZ T. Für $y \geqslant 1$ und $x \in\left[\frac{a}{b}, \frac{c}{d}\right]$ mit $\frac{a}{b}<\frac{c}{d}$ ist

$$
\grave{D}_{y}(x)=(b-d) x-(a-c) \text {. }
$$

Bemerkungen. (1) Der Graph von $\mathfrak{b}_{y}$ im $\boldsymbol{R}^{2}$ ist demnach die geradlinige Verbindung der Punkte

$$
\left(\frac{0}{1}, \frac{1}{1}\right), \ldots,\left(\frac{a}{b}, \frac{1}{b}\right), \ldots,\left(\frac{1}{1}, \frac{1}{1}\right)
$$

wenn

$$
\frac{0}{1}<\ldots<\frac{a}{b}<\ldots<\frac{1}{1}
$$

die Farey-Reilhe $\widetilde{F}_{y}$ bezeichnet.

(2) Für jedes $x$ aus $[0,1]$, das nicht zu $\mathfrak{F}_{y}$ gehört, ist $\left|\mathrm{D}_{y}^{\prime}(x)\right| \leqslant[y]-1$.

Beweis. Es sei $D_{y}^{*}:[0,1] \rightarrow \boldsymbol{R}$ definiert durch

$$
\grave{D}_{y}^{*}(x)=(b-d) x-(a-c) \quad \text { für } \quad x \in\left[\frac{a}{b}, \frac{e}{d}\right] \text { mit } \frac{a}{b}<\frac{c}{d} .
$$

Hilfssatz $2(\mathrm{i})$ zeigt, da $\mathrm{B}$ diese Definition konsistent ist. Wir beweisen $\mathfrak{D}_{y}=\mathfrak{D}_{y}^{*}$ für $y \in N$ induktiv. Im Fall $y=1$ ist $\mathfrak{o}_{y}(x)=1=\mathfrak{D}_{y}^{*}(x)$; es sei also $y \geqslant 2$.

(i) $x \in[0,1 / y]$ : Für $x=0$ ist $\mathrm{b}_{y}(0)=1=\mathrm{b}_{y}^{*}(0)$. Sonst ergibt sich $\mathrm{D}_{y}^{*}(x)=(1-y) x+1$, und denselben Wert liefert das ReduktionsLemma für $\mathrm{D}_{y}(x)$.

(ii) Für $x \in] 1 / y, 1 /(y-1)]$ folgt mit $1 / y<1 /(y-1)$ sofort $\mathrm{D}_{y}^{*}(x)=x$, und wegen $x \leqslant 1 /(y-1)$, also $y \leqslant 1+1 / x$, liefert das Reduktions-tumma zusammen mit $1 / y<x$, also $1-(y-1) x<x$, ebenfalls $\mathfrak{D}_{y}(x)$ $=\max \{x, 1-(y-1) x\}=x$.

(iii) Ist $x \in[1-1 /(y-1), 1]$, so folgt die Richtigkeit der Behauptung aus (i) und (ii) unter Benutzung der Symmetrieformeln

$$
\mathfrak{D}_{y}(x)=\mathrm{D}_{y}(1-x), \quad \mathrm{D}_{y}^{*}(x)=\mathrm{D}_{y}^{*}(1-x) .
$$

Die erste Formel besagt, daß $b_{y}$ invariant bleibt, wenn die Prnktefolge $(\{n x\}\}_{0 \leqslant n<[y]}$ am Punkt $1 / 2$ gespiegelt wird und dabei modalo 1 in die Folge $(\{n(1-x)\})_{0 \leqslant n<[y]}$ übergeht. Zur zweiten der beiden Gleichtngen ist zu bemerken: Aus $\frac{a}{b}<\frac{c}{d}$ und $x \in\left[\frac{a}{b}, \frac{c}{d}\right]$ folgen

$$
\frac{a-c}{a}<\frac{b-a}{b} \text { und } \quad 1-x \in\left[\frac{d-c}{d}, \frac{b-a}{b}\right]
$$

also ist

$$
\mathrm{D}_{y}^{*}(1-x)=(d-b)(1-x)-((d-c)-(b-a))=(b-a) x-(a-c)=\mathfrak{b}_{y}^{*}(x) .
$$

(iv) $x \in] 1 /(y-1), 1-1 /(y-1)[: a / b$ und $c / d$ bezeichne die benachbarten Brüche der Farey-Reihe $\mathfrak{F}_{y}$ mit $a / b<x \leqslant c / d$. Ohne Einschränkung darf

$$
\frac{a}{b}<x<\frac{e}{d}
$$

angenommen werden, denn für $x=c / d$ ist

$$
\mathrm{b}_{y}\left(\frac{c}{d}\right)=\frac{1}{d}=\mathrm{b}_{y}^{*}\left(\frac{c}{d}\right) \text {. }
$$

Wegen $x>1 /(y-1)$ sind $a$ und $c$ positiv. Für $n=[1 / x]$ folgt ans (3)

$$
\frac{d^{\prime}}{c}=\frac{d-n c}{c}<\left\{\frac{1}{x}\right\}<\frac{b-n a}{a}=\frac{b^{\prime}}{a}
$$

mit $d^{\prime} \mid c \geqslant 0$ und $b^{\prime} \mid a \leqslant 1$. Wäre nämlich $d^{\prime} \mid c<0$, so ergäbe (4)

$$
\frac{a}{c}<n<\frac{b}{a} \text {, also } \frac{a}{b}<\frac{1}{n}<\frac{c}{d} \quad \text { mit } \quad n \leqslant \frac{1}{a}<y-1 \text {, }
$$

im Widerspruch zu $a / b<c / d$. Und ans $b^{\prime} \mid a>1$ wïrde mit (4) $d / c<n+1$ $<b / a$ folgen, also

$$
\frac{a}{b}<\frac{1}{n+1}<\frac{c}{d} \quad \text { mit } \quad n+1<y .
$$

Ferner können wir

$$
b>d
$$

annehmen. $b=d$ führt wegen $b c-a d=1$ nämlich auf $b=d=1$ and somit auf den Fall $y=1$. Ist aber $b<d$, reicht es wegen der unter (iii) bewiesenen Symmetrieformeln, die Behauptung für $x^{*}=1-x$ statt für $x$ zu beweisen, und dann ist

$$
\frac{a-c}{d}<x^{*}<\frac{b-a}{b} \text { mit } \quad \frac{d-e}{d}<\frac{b-a}{b}
$$

and $b^{*}=a>b=d^{*}$. SchlieBlich gilt

$$
\frac{d^{\prime}}{e} \underset{y^{\prime}}{<} \frac{b^{\prime}}{a} \quad \text { mit } \quad y^{\prime}=x\left(y-\left[\frac{1}{x}\right]\right)+1 \text {. }
$$

Denn zunächst ist nach $(3) a<x b \leqslant x y \leqslant y^{\prime}$ und $o \leqslant a$, also auch $o \leqslant y^{\prime}$, da aus $c>a$ mit $a d+1=b c$ sofort $a d \geqslant a b$ und damit $d \geqslant b$ folgen würde, im Widerspruch zu (5). Es bleibt zu zeigen, daß fïr jeden Bruch u/v mit 
$d^{\prime} / c<u / v<b^{\prime} / a$ die Ungleichung $v>\left[y^{\prime}\right]$ folgt. Das geschieht, indem sulzessiv

$$
v \geqslant a+e \geqslant \frac{c}{d} y+\frac{c-1}{d}>y^{\prime}
$$

bewiesen wird. Aus $d^{\prime} / c<u / v<b^{\prime} / a$ folgen $d^{\prime} v+1 \leqslant c u$ und $u a \leqslant v b^{\prime}-1$, also $a \bar{d}^{\prime} v+a \leqslant a c u \leqslant c v b^{\prime}-c$, und das zeigt

$$
a+c \leqslant v\left(c b^{\prime}-a \bar{d}^{\prime}\right)=v(c(b-n a)-a(d-n c))=v(b c-a d)=v
$$

Wegen

$$
\frac{a}{b}<\frac{c}{d} \quad \text { und } \quad \frac{a}{b}<\frac{a+c}{b+d}<\frac{c}{d}
$$

muß $b+d \geqslant y+1$ sein, und damit $(a+c) d+1=a d+1+c d=b c+c d$ $\geqslant a(y+1)$, also

$$
a+e \geqslant \frac{e}{d} y+\frac{c-1}{d}
$$

Die Ungleichung $[d / c]+1>d / c$ führt bei Multiplikation mit $c$ auf $c([d / c]+1) \geqslant \ddot{d}+1$, also auf

$$
\frac{c}{d}\left(\left[\frac{d}{c}\right]+1\right) \geqslant 1+\frac{1}{d}
$$

Außexdem ist

$$
\left(\frac{c}{d}-x\right)\left(y-\left[\frac{d}{c}\right]\right)>0
$$

denn aus $[d / c] \geqslant y$ würde $x<c / d \leqslant 1 / y$ folgen. Addition von (7) und (8). ergibt nun

$$
\frac{c}{d}(1+y)>1+\frac{1}{d}+x\left(y-\left[\frac{d}{c}\right]\right)
$$

also

$$
\frac{e}{a} y+\frac{o-1}{a}>x\left(y-\left[\frac{d}{c}\right]\right)+1 \geqslant x\left(y-\left[\frac{1}{x}\right]\right)+1=y^{\prime},
$$

und damit ist (6) bewiesen.

Nun kann wegen $y^{\prime} \leqslant x y+1<\left(1-\frac{1}{y-1}\right) y+1<y$ die Induktionsvoraussetzung auf $\left[y^{\prime}\right]$ und die Stelle $\{1 / x\}$ angewandt werden. Das liefert unter Beachtung von (4) und (6)

$$
\delta_{y^{\prime}}(\{1 / x\})=\delta_{y^{\prime}}^{*}(\{1 / x\})=(c-a)\{1 / x\}-\left(d^{\prime}-b^{\prime}\right) .
$$

Mit der Gleichung

$$
\mathrm{D}_{y}(x)=x \cdot \mathrm{D}_{y^{\prime}}(\{1 / x\})
$$

des Reduktions-Lemmas exgibt sich also

$$
\mathrm{D}_{y}(x)=x\left((c-a)\left(\frac{1}{x}-n\right)-\left(d^{\prime}-b^{\prime}\right)\right)=(b-d) x-(a-c)=\mathfrak{D}_{y}^{*}(x) .
$$

SATZ 8.

$$
\int_{0}^{1} D_{y}(x) d x=0\left(\frac{\log y}{y}\right)
$$

Beweis. Wir zeigen, daß $\frac{\log y}{y}$ die genaue Größenordnung von $\int_{0}^{1} \mathrm{D}_{v}(x) d x$ ist. Für $y=N \geqslant 2, b>1$ und $\frac{r}{s}<_{N} \frac{a}{b}<_{N} \frac{u}{v}$ liegt $a / b$ im FareyBogen

$$
F_{a / b}=\left[\frac{r+a}{s+b}, \frac{a+u}{b+v}\right]
$$

and für dessen beide Teile

$$
F_{a \mid b}^{1}=\left[\frac{r+a}{s+b}, \frac{a}{b}\right] \quad \text { und } \quad F_{a / b}^{2}=\left[\frac{a}{b}, \frac{a+u}{b+v}\right]
$$

gilt nach Eilfssatz 2(ii)

$$
\text { (9) } \quad \frac{1}{2 b N}<\mu\left(F_{a / b}^{i}\right)<\frac{1}{b N} \quad(i=1,2) \text {. }
$$

In dem Randpunkt $a / b$ von $F_{a l b}^{i}$ nimmt $D_{N}(x)$ den Wert $1 / b$ an; ferner

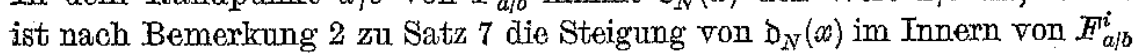
stets kleiner als $N$. Das ergibt für alle $x \in F_{a / b}$

$$
\frac{1}{b}-\left|x-\frac{a}{b}\right| N \leqslant \grave{D}_{N}(x) \leqslant \frac{1}{b}+\left|x-\frac{a}{b}\right| N .
$$

Daraus folgt mit (9)

$$
\begin{aligned}
& \int_{F a / b} D_{N}(x) d x \leqslant \int_{\frac{a}{b}-\frac{1}{b N}}^{\tilde{b}^{+}+\frac{1}{b N}}\left(\frac{1}{b}+\left|x-\frac{a}{b}\right| N\right) d x \leqslant \frac{3}{b^{2} N}, \\
& \int_{F=b} \mathfrak{D}_{N}(x) d x \geqslant \int_{\frac{a}{b}-\frac{1}{2 b N}}^{\frac{a}{b}+\frac{1}{2 b N}}\left(\frac{1}{b}-\left|x-\frac{a}{b}\right| N\right) d x \geqslant \frac{3}{4 b^{2} N}
\end{aligned}
$$


Summation über alle Brüche $a / b$ der Farey-Reihe $\mathfrak{F}_{N}$ mit $b>1$ ergibt

(10) $\quad \frac{3}{4 N} \sum_{b=2}^{N} \frac{\varphi(b)}{b^{2}} \leqslant \int_{0}^{1} \mathfrak{b}_{N}(x) d x \leqslant \frac{3}{N} \sum_{b=2}^{N} \frac{\varphi(b)}{b^{2}}+\frac{2}{N}$.

Ans der Formel

$$
\sum_{b=1}^{N} \varphi(b)=\frac{3}{\pi^{2}} N^{2}+O(N \log N)
$$

kommt bei Abelscher partieller Summation

$$
\sum_{b=1}^{N} \frac{\varphi(b)}{b^{2}}=\frac{6}{\pi^{2}}(\log N)+O(\mathcal{1})
$$

und mit (10) und einer positiven Konstante $e$ führt das auf

$$
\frac{9}{2 \pi^{2}} \frac{\log N}{N}-\frac{c}{N} \leqslant \int_{0}^{1} D_{N}(x) d x \leqslant \frac{18}{\pi^{2}} \frac{\log N}{N}+\frac{e}{N} .
$$

\section{Teilbarkeit durch $d$}

$\mathrm{SATZ}_{\triangle \mathrm{T}}$ 9. Fü̈ Funktionen f aus $\mathscr{F}_{\text {pol }}$ vom Grad $k \geqslant 2$, natürliche Zahlen $\vec{*}$ und $\mathscr{P}=d \boldsymbol{N}$ ist mit einer von $d$ unabhängigen Konstante in $O_{f}$

$$
\int_{0}^{1}\left|S_{N}^{\text {nat }}(x)-\frac{N}{d}\right| d x=\sqrt{d} O_{f}\left(N^{3 / 4} \log N\right)
$$

Wie in Teil 3 folgt daraus das

CoRoLxaR. Für Funktionen $f$ wie in Satz 9 und alle natürlichen Zahlen d ist $D_{\mathrm{nat}}^{\mathrm{nat}}(d \boldsymbol{N}, f)=1$.

Bemerkung. Im Zusammenhang mit diesem Ergebnis stehen Arbeiten von Rieger [26] und Wolke [30], in denen für $1<k<2$ die Anzahl der $\left[n^{k}\right]$ ermittelt wird, die kongruent $m$ modulo $d$ sind. Deshouillers hat in [5] den Fall nichtganzer Exponenten $k>1$ behandelt.

Beweis von Satz 9. $f$ und $d$ seien rorgegeben. Dabei kann angenommen werden, daß $f$ Forderung (F1) erfüllt. Füir dieses $f$ wird zunächst gezeigt:

HUFESATZ 3. (i) Für alle natürlichen Zahlen $n$ definiert

$$
g_{n}(x)=\frac{f(n+\infty)-f(n)}{f(n+1)-f(n)}
$$

eine streng monoton wachsende Frunktion von $[0,1]$ arf $[0,1]$ mit

$$
\frac{1}{g_{n}^{\prime}(x)}=1+O_{f}\left(\frac{1}{n}\right)
$$
stets

(ii) Mit $h_{m, n}=g^{m \circ} \circ g_{n}^{-1}$ und $H_{m, n}(x)=h_{m, n}^{-1}(x)-x$ gitt fiir $m \leqslant n$

$$
H_{m, n}(x)=O_{f}\left(\frac{1}{m}\right) \quad \text { und } \quad H_{m, n}^{\prime}(x)=O_{f}\left(\frac{1}{m}\right) .
$$

Beweis. (i) folgt ans

$\frac{1}{g_{n}^{\prime}(x)}=\frac{f(n+1)-f(n)}{f^{\prime}(n+x)} \quad$ and $\quad \frac{f^{\prime}(n)}{f^{\prime}(n+1)} \leqslant \frac{f(n+1)-f(n)}{f^{\prime}(n+x)} \leqslant \frac{f^{\prime}(n+1)}{f^{\prime}(n)}$.

(ii) Aus $H_{m, n}\left(g_{m}(x)\right)=g_{n}(x)-g_{m}(x)$ folgt durch Differenzieren

$$
H_{m, n}^{\prime}\left(g_{m}(x)\right)=\frac{g_{n}^{\prime}(x)}{g_{m}^{\prime}(x)}-1=\frac{f^{\prime}(n+x)}{f^{\prime}(m+x)} \frac{f(m+1)-f(m)}{f(n+1)-f(n)}-1 .
$$

Da $f^{\prime}$ monoton steigt, ist

$\frac{f^{\prime}(n)}{f^{\prime}(n+1)} \frac{f^{\prime}(m)}{f^{\prime}(m+1)} \leqslant \frac{f^{\prime}(n+x)}{f^{\prime}(m+x)} \frac{f(m+1)-f(m)}{f(n+1)-f(n)} \leqslant \frac{f^{\prime}(n+1)}{f^{\prime}(m)} \frac{f^{\prime}(m+1)}{f^{\prime}(n)}$,

und mit

folgt

$$
\frac{f^{\prime}(y+1)}{f^{\prime}(y)}=1+O_{f}\left(\frac{1}{y}\right) \quad \text { und } \quad m \leqslant n
$$

$$
H_{m, n}^{\prime}\left\{g_{m}(x)\right)=\left(1+o_{f}\left(\frac{1}{m}\right)\right)\left(1+o_{f}\left(\frac{1}{n}\right)\right)-1=o_{f}\left(\frac{1}{m}\right) .
$$

In Anbetracht der Bijektivität von $g_{m}$ ist das die zweite Behauptung in (ii). Aus dieser folgt mit $H_{m, n}(0)=0$ sofort die erste.

Nun wird

$$
\begin{aligned}
& \int_{0}^{1} S_{N}^{\text {nat }}(d \mathcal{N} ; x)^{2} d x=\sum_{m=1}^{N} \sum_{n=m}^{N}\left(2-\delta_{m, n}\right) I^{m, n}, \\
& I^{m, n}=\int_{0}^{1} \chi_{d N}([f(m+x)]) \chi_{d N}([f(n+x)]) d x,
\end{aligned}
$$

abgeschätzt. Für $x \in[0,1]$ ist

$$
\chi_{d N}([f(n+x)])= \begin{cases}1 & \text { für } \quad x \in\left[f^{-1}(d r)-n, f^{-1}(d r+1)-n[, r \in N,\right. \\ 0 & \text { sonst. }\end{cases}
$$

$[0,1]$ wird nur von den Intervallen

$$
\left[f^{-1}(d r)-n, f^{-1}(d r+1)-n\left[\text { mit } \quad \frac{f(n)-1}{d}<r \leqslant \frac{f(n+1)}{d}\right.\right.
$$


geschnitten. Werden von diesen jene Intervalle unterschlagen, die 0 oder 1 enthalten, definieren wir also auf $[0,1]$ mit

$$
\begin{gathered}
\left.\left.M_{n}=N \cap\right] \frac{f(n)}{d}, \frac{f(n+1)-1}{d}\right] \\
\chi_{n}^{(1)}(x)= \begin{cases}1 & \text { für } \quad x \in\left[f^{-1}(d r)-n, f^{-1}(d r+1)-n\left[, \quad r \in M_{n},\right.\right. \\
0 & \text { sonst, }\end{cases}
\end{gathered}
$$

so folgt für $m \leqslant n$

$$
I^{m, n}=\int_{0}^{1} x_{m}^{(1)}(x) \chi_{n}^{(1)}(x) d x+O_{f}\left(\frac{1}{m}\right),
$$

denn nach (1) in Teil 1 ist die Länge der eventuell unterschlagenen Intervalle beschränkt durch $o_{f}\left(\frac{1}{m}\right)$. Ferner ist

$$
\left|I_{n}\right|=\frac{f(n+1)-f(n)}{d}+O(1) \text {. }
$$

Für $\chi_{n}^{(2)}=\chi_{n}^{(1)} \circ g_{n}^{-1}$ und $n \geqslant m$ folgt mit Hilfssatz 3(i) aus (2).

$$
\text { (4) } \quad \begin{aligned}
I^{m, n} & =\int_{0}^{1} x_{m}^{(2)}\left(g_{m}(x)\right) \chi_{n}^{(2)}\left(g_{n}(x)\right) d x+O_{f}\left(\frac{1}{m}\right) \\
& =\int_{0}^{1} \chi_{m}^{(2)}\left(g_{m} g_{n}^{-1}(y)\right) \chi_{n}^{(2)}(y) \frac{d y}{g_{n}^{\prime} g_{n}^{-1}(y)}+O_{f}\left(\frac{1}{m}\right) \\
& =\int_{0}^{1} \chi_{m}^{(2)}\left(h_{m, n}(x)\right) \chi_{n}^{(2)}(x) d x+O_{f}\left(\frac{1}{m}\right) .
\end{aligned}
$$

Dabei ist mit den Abkürzungen $e_{n}=f(n+1)-f(n)$ und $r_{n}=d r-f(n)$

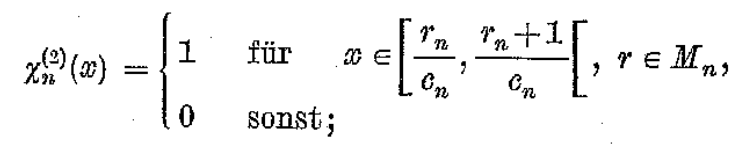

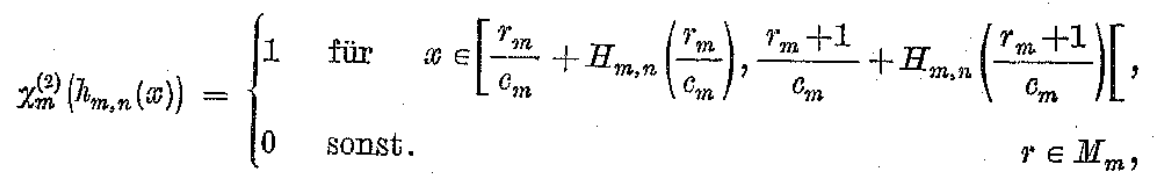
$\chi_{m}^{(2)} \circ h_{m, n}$ wird modifiziert zu

$x_{m, n}(x)= \begin{cases}1 & \text { für } \quad x \in\left[\frac{r_{m}}{c_{m}}+H_{m, n}\left(\frac{r_{m}}{c_{m}}\right), \frac{r_{m}+1}{c_{m}}+H_{m_{n} n}\left(\frac{r_{m}}{c_{m}}\right)\left[, r \in M_{m},\right.\right. \\ 0 & \text { sonst. }\end{cases}$
Aus Hilfssatz 3(ii) folgt

$$
H_{m, n}\left(\frac{r_{m}+1}{c_{m}}\right)-H_{m, n}\left(\frac{r_{m}}{e_{m}}\right)=O_{f}\left(\frac{1}{m e_{m}}\right)
$$

also ist $\chi_{m}^{(2)}\left(h_{m, n}(x)\right)=\chi_{m, n}(x)$ richtig außerhalb einer Menge rom $\mathrm{MaB}$ $o_{f}\left(\frac{1}{m c_{m}}\left|M_{m}\right|\right)$, und mit (4) und (3) erhalten wir

$$
\begin{aligned}
I^{m, n} & =\int_{0}^{1} \chi_{m, n}(x) \chi_{n}^{(2)}(x) d x+O_{f}\left(\frac{1}{m}\right) \\
& =\int_{-\infty}^{+\infty} \chi_{m, n}(x) \chi_{n}^{(2)}(x) d x+O_{f}\left(\frac{1}{m}\right),
\end{aligned}
$$
wenn $\chi_{m, n}$ und $\chi_{n}^{(2)}$ außerhalb $[0,1]$ fortgesetzt werden durch $\chi_{m, n}(x)$
$=\chi_{n}^{(2)}(x)=0$.

An der Beschreibung von $\chi_{n}^{(2)}$ in (5) läßt sich ablesen, daß

$$
\sum_{t=0}^{d-1} \chi_{n}^{(2)}\left(x-\frac{t}{c_{n}}\right)
$$

den Wert 1 annimmt für alle $w$ zwischen $O\left(d / o_{n}\right)$ und $1+O\left(d / c_{n}\right)$ und den Wert 0 sonst. Deshalb ist

$$
\sum_{t=0}^{d-1} \int_{-\infty}^{+\infty} \chi_{n}^{(2)}\left(x-\frac{t}{c_{n}}\right) \chi_{m, n}(x) d s=\int_{-\infty}^{+\infty} \chi_{m, n}(x) d x+O\left(\frac{d}{c_{n}}\right) .
$$

Andererseits folgt aus der Definition von $\chi_{m, n}$ und aus (3)

Also wird

$$
\int_{-\infty}^{+\infty} \chi_{m, n}(x) d s=\frac{1}{c_{m}}\left|M_{m}\right|=\frac{1}{d}+o\left(\frac{1}{c_{m}}\right) .
$$

$$
\sum_{i=0}^{d-1} \int_{-\infty}^{+\infty} \chi_{n}^{(2)}\left(x-\frac{t}{e_{n}}\right) \chi_{m, n}(x) d x=\frac{1}{d}+O\left(\frac{d}{e_{m}}\right)
$$

Es seien $a$ und $b$ niehtnegative ganze Zahlen. Aus

$$
\int_{-\infty}^{+\infty}\left|\chi_{n}^{(2)}\left(x-\frac{t}{c_{n}}\right)-\chi_{n}^{(2)}\left(x-\frac{t}{c_{n}}-\frac{a d}{c_{n}}\right)\right| d x=\frac{2 a}{c_{n}}
$$

folgt

(8) $\int_{-\infty}^{+\infty}\left(\chi_{n}^{(2)}\left(x-\frac{t}{e_{n}}\right)-\chi_{n}^{(2)}\left(x-\frac{t}{e_{n}}-\frac{a d}{c_{n}}\right)\right) \chi_{m, n}(x) d x=o\left(\frac{a}{c_{n}}\right)$. 


\section{Ferner ist}

(9)

$$
\int_{-\infty}^{+\infty}\left|\chi_{m_{2}, n}(x)-\chi_{m, n}\left(x-\frac{b d}{c_{m}}\right)\right| d x=O\left(\frac{b d}{m}\right) .
$$

Denn nach Definition von $\chi_{m, n}$ ist $\chi_{m, n}(x)=1$ genau für die Zahlen $x$ in einem der Intervalle

$$
J_{r}=\left[\frac{r_{m}}{c_{m}}+H_{m, n}\left(\frac{r_{m}}{c_{m}}\right), \frac{r_{m}+1}{c_{n}}+H_{n, n}\left(\frac{r_{m}}{c_{m}}\right)\left[, \quad r \in M_{m} .\right.\right.
$$

Hingegen ist $\chi_{m, n}\left(x-\frac{b d}{c_{m}}\right)=1$ genau für die $x$ in

$$
J_{s}^{\prime}=\left[\frac{s_{m}}{c_{m}}+H_{m, n}\left(\frac{s_{m}}{c_{m}}\right)+\varepsilon_{s}, \frac{s_{m}+1}{c_{m}}+H_{m, n}\left(\frac{s_{m}}{c_{m}}\right)+\varepsilon_{s}\left[, \quad s-b \in M_{m},\right.\right.
$$

mit

$$
\varepsilon_{s}=H_{m, n}\left(\frac{d(s-b)-f(m)}{c_{m}}\right)-H_{m, n}\left(\frac{d s-f(m)}{c_{m}}\right)=O\left(\frac{b d}{m c_{m}}\right)
$$

Der $O$-Term rechts in (9) setzt sich also zusammen aris höchstens $\left|M_{m}\right|$ Fehlern $O\left(\frac{b d}{m c_{m}}\right)$ und den $2 b$ Intervallängen $\frac{1}{c_{m}}$ der $J_{r}$ mit $r-b \notin M_{m}$ und der $J_{s}^{\prime}$ mit $s \dot{\leftrightarrow} M_{m}$.

Aus (8) and (9) folgt für alle ganzen $t$ zwischen 0 und $d \rightarrow 1$ :

(10) $\int_{-\infty}^{+\infty} \chi_{n}^{(2)}\left(x-\frac{t}{c_{n}}\right) \chi_{m, n}(x) d x$

$$
\begin{aligned}
& =\int_{-\infty}^{+\infty} \chi_{n}^{(2)}\left(x-\frac{t}{c_{n}}-\frac{a d}{e_{n}}\right) \chi_{m, n}(x) d x+o\left(\frac{a}{c_{n}}\right) \\
& =\int_{-\infty}^{+\infty} \chi_{n}^{(2)}\left(x-\frac{t}{c_{n}}-\frac{a d}{c_{n}}\right) \chi_{m, n}\left(x-\frac{b d}{c_{m}}\right) d x+o\left(\frac{a}{c_{n}}+\frac{b d}{m}\right) \\
& =\int_{-\infty}^{+\infty} \chi_{n}^{(2)}(x) \chi_{m, n}\left(x+\frac{t}{c_{n}}+\frac{a d}{c_{n}}-\frac{b d}{c_{m}}\right) d x+o\left(\frac{a}{c_{n}}+\frac{b d}{m}\right) .
\end{aligned}
$$

Mit der Funktion $D_{y}$ ans Teil 4 ist

$$
\begin{aligned}
\min \left\{\mid \frac{t}{c_{n}}+\frac{a d}{c_{n}}\right. & \left.-\frac{b d}{c_{m}} \mid ; a, b \in \boldsymbol{N}_{0}, b \leqslant \sqrt{m}\right\} \\
& \leqslant \frac{d}{c_{n}} \sup _{x \in[0,1]} \min \left\{\left|x+a-b \frac{c_{n}}{c_{m}}\right| ; a, b \in \mathbf{N}_{0}, b \leqslant \sqrt{m}\right\} \\
& \leqslant \frac{d}{c_{n}} b_{\sqrt{m}}\left(\frac{c_{n}}{c_{m}}\right) .
\end{aligned}
$$

Werden also $a_{t} \in N_{0}$ und $b_{i} \leqslant \sqrt{m}$ so gewälltt, da $B$ für

$$
\begin{gathered}
\varepsilon_{t}=\frac{t}{c_{n}}+\frac{a_{t} d}{c_{n}}-\frac{b_{t} d}{c_{m}} \\
\left|\varepsilon_{t}\right| \leqslant \frac{d}{c_{n}} \mathfrak{b}_{v^{\prime}}\left(\frac{c_{n}}{c_{m}}\right)
\end{gathered}
$$

gilt, so folgt mit (10) wegen $\frac{a_{t}}{a_{n}} \leqslant \frac{b_{t}}{c_{m}}+\frac{\varepsilon_{t}}{d} \leqslant \frac{1}{\sqrt{m}}$

(12) $\int_{-\infty}^{+\infty} \chi_{n}^{(2)}\left(x-\frac{t}{c_{n}}\right) \chi_{m, n}(x) d x=\int_{-\infty}^{+\infty} \chi_{n}^{(2)}(x) \chi_{m, n}\left(x+\varepsilon_{t}\right) d x+o\left(\frac{d}{\sqrt{m}}\right)$.

Aus

$$
\int_{-\infty}^{+\infty} \chi_{n}^{(2)}(x)\left(\chi_{m, n}\left(x+\varepsilon_{t}\right)-\chi_{m, n}(x)\right) d x=O\left(\left|\varepsilon_{t}\right|\left|M M_{m}\right|\right)
$$

ergibt sich mit (12), (11) und (3)

$$
\begin{aligned}
\int_{-\infty}^{+\infty} \chi_{n}^{(2)}\left(x-\frac{t}{c_{n}}\right) & \chi_{m, n}(x) d x \\
& =\int_{-\infty}^{+\infty} \chi_{n}^{(2)}(x) \chi_{m, n}(x) d x+o\left(\frac{d}{\sqrt{n}}+\frac{c_{m}+d}{c_{n}} b_{v m}\left(\frac{c_{n}}{c_{m}}\right)\right) .
\end{aligned}
$$

Addition dieser Gleichungen für $0 \leqslant t \leqslant d-1$ und anschließende Division durch $d$ liefert unter Beachtung von $(6)$ und $(7)$.

$$
I^{m, n}=\frac{1}{d^{2}}+O_{f}\left(\frac{d}{\sqrt{m}}+\frac{c_{m}}{e_{n}} \triangleright_{\sqrt{m}}\left(\frac{c_{n}}{c_{m}}\right)\right)
$$

Das wird in (1) eingesetzt:

(13) $\left.\int_{0}^{1} S_{N}^{\text {nat }}(x)^{2} d x=\frac{N^{2}}{d^{2}}+O_{f}\left(\sum_{m=1}^{N} \sum_{n=m}^{N}\left(\frac{d}{\sqrt{m}}+\frac{c_{m}}{c_{n}} \searrow_{/}-\frac{c_{n}}{c_{m}}\right)\right)\right)$.

Nun schätzen wir den $O$-Term von (13) ab:

$$
\begin{aligned}
R(N) & =\sum_{m=1}^{N} \sum_{n=m}^{N} \frac{c_{m}}{c_{n}} \mathrm{~b}_{V^{\prime}}\left(\frac{c_{n}}{c_{m}}\right) \\
& \leqslant \sum_{m=1}^{N} \sum_{1 \leqslant i \leqslant c_{N} / c_{m}}\left(\frac{1}{i} \cdot \sum_{i c_{m} \leqslant c_{n}<(i+1) c_{m}} \grave{b}_{\sqrt{m}}\left(\frac{c_{n}}{c_{m}}\right)\right) .
\end{aligned}
$$


Zur Behandlung der innersten Summe in (14) werden die nach (F1) streng monoton wachsende Funktion

$$
h(y)=\frac{1}{c_{m}}(f(y+1)-f(y))
$$

und die Eulersche Summenformel mit $B_{0}(y)=y-[y]-\frac{1}{2}$ benutzt:

$$
\begin{aligned}
& \sum_{i=1} \mathfrak{d}_{\sqrt{m}}\left(\frac{c_{n}}{c_{m}}\right) \leqslant \sum_{c^{-1}(i) \leqslant n \leqslant n_{n}<(i+1) c_{m}} \grave{d}_{\sqrt{m}}(h(n)) \\
& \leqslant \int_{h^{-}(i)}^{h^{-1}(i+1)}\left(\widehat{D}_{V \bar{m}} \circ h\right)(y) d y+\int_{h^{-1}(i)}^{h^{-1}(i+1)} B_{0}(y)\left(\bigsqcup_{V \bar{m}} \circ h\right)^{\prime}(y) d y- \\
& -\delta_{\sqrt{m}}(i+1) B_{0}\left(h^{-1}(i+1)\right)+\frac{1}{2} \delta_{\sqrt{m}}(i) \\
& \leqslant \frac{1}{h^{\prime} h^{-1}(i+\Theta)} \int_{0}^{1} D_{\sqrt{m}}(x) d x+\int_{0}^{1}\left|D_{V \bar{m}}^{\prime}(x)\right| d x+1 .
\end{aligned}
$$

Weil auf Grund ron (F1) neben $h$ anch $h^{\prime}$ monoton steigt, ist

und daher

$$
h^{\prime}\left(h^{-1}(i+\Theta)\right) \geqslant h^{\prime} h^{-1}(1)=h^{\prime}(m)
$$

$$
\frac{1}{h^{\prime} h^{-1}(i+\Theta)}=O_{f}(m)
$$

Satz 8 und Bemerkung 2 zu Satz 7 ergeben also

$$
\sum_{\substack{n \\ i c_{m} \leqslant c_{n}<(i+1) c_{m}}} \grave{b}_{\sqrt{m}}\left(\frac{c_{n}}{c_{m}}\right)=O_{f}(m) O\left(\frac{\log m}{\sqrt{m}}\right)+O(\sqrt{m})=O_{f}(\sqrt{m} \log m) .
$$

Damit folgt aus (14)

$$
R(N)=O_{f}\left(\sum_{m=1}^{N} \sum_{i=1}^{c_{N}} \frac{1}{i} \sqrt{m} \log m\right)=O_{f}\left(N^{3 / 2} \log ^{2} N\right),
$$

und das in Verbindung mit (13) zeigt

$$
\int_{0}^{1} S_{N}^{\text {nat }}(x)^{2} d x=\frac{N^{2}}{d^{2}}+d O_{f}\left(N^{3 / 2} \log ^{2} N\right)
$$

Aus Lemma 1 folgt

(16)

$$
\int_{0}^{1} S_{N}^{\text {nat }}(d N ; x) d x=\frac{N}{d}+O_{f}(\log N)
$$

und aas (15) und (16) kommt mit dem Corollar zu Lemma 3

$$
\int_{0}^{1}\left|S_{N}^{\text {nat }}(x) \frac{d}{N}-1\right| d x=d^{3 / 2} O_{f}\left(N^{-1 / 4} \log N\right)
$$

6. Anwendung des Selberg-Siebes. Mit dem Selbergschen Siebverfahren haben Halberstam und Richert ([9], S. 162) bewiesen: Ist $f$ aus $\boldsymbol{Z}[y]$, irreduzibel und mit positivem Leitkoeffizienten, bezeichnet $\varrho(p)$ für Primzahlen $p$ die Anzahl der Lösungen von $f(n) \equiv 0 \bmod p$ und ist stets $\varrho(p)<p$, so gilt

$$
D_{\mathrm{nat}}^{\mathrm{nat}}(P, f ; N)(0) \leqslant 2 \prod_{p \in P}\left(1-\frac{\varrho(p)-1}{p-1}\right) \cdot\left(1+O_{f}\left(\frac{\log \log 3 N}{\log N}\right)\right) .
$$

Hier soll gezeigt werden:

SATZ 10. Fïr Eunktionen $f$ aus $\mathscr{F}_{\text {pol }}$ vom $G_{r a d} k \geqslant 2$ ist

$$
\limsup _{N \rightarrow \infty} D_{\text {nat }}^{\mathrm{nat}}(P, f ; N) \leqslant \frac{10}{3} \pi^{2}
$$

Beweis. Wir gehen aus von Satz 9. Für $\varepsilon>0$ sei

$$
A(N, d)=\left\{x \in I^{1} ;\left|S_{N}^{\text {nat }}(d, N ; x)-N\right| d \mid \geqslant N^{1-\varepsilon}\right\} \text {. }
$$

Dann ist

$$
\mu(A(N, d)) N^{1-8} \leqslant \int_{0}^{1}\left|S_{N}^{\text {nat }}(d N ; x)-N\right| d \mid d x \leqslant c_{f} \sqrt{d} N^{3 / 4} \log N
$$

also

$$
\mu\left(A\left(N, d_{s}\right)\right)=O_{f}\left(\sqrt{d} N^{s-1 / 4} \log N\right)
$$

Mit einem $\tau>0$ sei

$$
A(N)=\bigcup_{1 \leqslant d \leqslant N^{2 \tau}} A(N, d)
$$

Demnach wird

(1) $\quad \mu(A(N))=O_{f}\left(\sum_{1 \leqslant \bar{d} \leqslant N^{2 x}} \sqrt{d} N^{g-1 / 4} \log N\right)=O_{f}\left(N^{e+3 x-1 / 4} \log N\right)$,

und für die natürlichen Zahlen $d \leqslant N^{2 \tau}$ und alle $x$ aus $A^{\prime}(N)=I^{1}-A(N)$ haben wir den Sieb-Ansatz

$$
\begin{gathered}
S_{N}^{\text {nat }}(d N ; x)=\sum_{\substack{n=1 \\
d \mid[f(n+x)]}}^{N} 1=\frac{N}{d}+R(N, d) \\
R(N, d)=O\left(N^{1-s}\right)
\end{gathered}
$$


Bezeichnet $S(\tau, N, x)$ die Anzahl der Elemente von $[f(n+x)]_{1 \leqslant n \leqslant N}$, die durch keine PrimzahI $p \leqslant N^{\tau}$ teilbar sind, so ist für alle $x$ aus $\mathcal{A}^{\prime}(N)$ nach einem Satz von Selberg (Halberstam-Roth [10], S. 213)

$$
S(\tau, N, x) \leqslant \frac{N}{Q}+\sum_{d_{1}, d_{2} \in D}\left|\Lambda_{d_{1}} \Lambda_{d_{2}} R\left(N, \mathrm{~kg} \cdot\left(d_{1}, d_{2}\right)\right)\right|
$$

mit

$$
\begin{aligned}
D & =\left\{d \in N ; d \leqslant N^{\tau}, d \text { quadratfrei }\right\} \\
g(d) & =d \prod_{\substack{p \in P \\
p \mid d}}\left(1-\frac{1}{p}\right) \\
Q & =\sum_{d \in D} \frac{1}{g(d)} \\
\Lambda_{d} & =\frac{\mu(d) d}{Q} \sum_{\substack{\delta \in D \\
d \mid \delta}} \frac{1}{g(\delta)},
\end{aligned}
$$

und hier bezeichnet $\mu$ die Möbiussche Funktion.

Für alle $d_{1}, d_{2}$ aus $D$ ist $\operatorname{kgV}\left(d_{1}, d_{2}\right) \leqslant N^{2 \tau}$; also folgt aus Formel (2)

$$
R\left(N, \operatorname{kg} \nabla\left(d_{1}, d_{2}\right)\right)=O\left(N^{1_{-}-8}\right) .
$$

Nan werden $\Lambda_{d}$ und $Q$ abgeschätzt:

$(5)$

$$
\begin{aligned}
\left|\Lambda_{d}\right| & =\frac{d}{Q} \sum_{\substack{\left.\delta \in D \\
d\right|^{\delta}}} \frac{1}{g(\delta)}=\frac{d}{g(d) Q} \sum_{\substack{\delta \in D \\
d \mid \delta}} \frac{g(d)}{g(\delta)}=\frac{d}{g(d) Q} \sum_{\substack{\delta \leq D \\
d \mid \delta}} \frac{1}{g(\delta / d)} \\
& \leqslant \frac{d}{g(d) Q} \sum_{d^{\prime} \in D} \frac{1}{g\left(d^{\prime}\right)} \leqslant \frac{\dot{d}}{g(d)}=\prod\left(1-\frac{1}{p}\right)^{-1} \leqslant \sqrt{\prod_{p \mid d}\left(1-\frac{1}{p}\right)^{-1}} \\
& =O(\log N) .
\end{aligned}
$$

Die Anzahl der quadratfreien Zahlen $\leqslant y$ ist $\frac{6}{\pi^{2}} y+O(\sqrt{y})$. Daraus folgt bei Abelscher partieller Summation mit einem $c>0$

$$
Q \geqslant \frac{6 \tau}{\pi^{2}} \log N-c
$$

Bei Einsetzung von (4), (5) und (6) in (3) ergibt sich für alle $x$ aus $A^{\prime}(N)$

$$
S(\tau, N, x) \leqslant \frac{\pi^{2} N}{6 \tau \log N-0^{b}}+O\left(N^{1+2 \tau-8} \log ^{2} N\right)
$$

Jede der Primzahlen in $[f(n+x)]_{1 \leqslant n \leqslant N}$ ist entweder $\leqslant N^{*}-$ und davon gibt es höchstens $N^{\tau}$ - oder aber sie ist $>N^{\tau}$ und dann sicher durch keine Primzahl $\leqslant N^{\tau}$ teilbar. Demnach folgt mit (7) für alle $x$ aus $A^{\prime}(N)$

$$
S_{N}^{\text {nat }}(P ; x) \leqslant \frac{\pi^{2} N}{6 \tau \log N-c^{\prime}}+O\left(N^{z}+N^{1+2 \tau-8} \log ^{2} N\right) .
$$

Wählen wir nun für große natürliche Zahlen $M \geqslant M_{0}$

$$
\tau=\frac{1}{20}-\frac{1}{M} \quad \text { und } \quad \varepsilon=\frac{1}{10}-\frac{1}{M},
$$

so folgt für $N \geqslant N\left(M_{0}, e^{\prime}\right)$ mit positiven Konstanten $c_{1}$ und $c_{2}$

(8) $S_{N}^{\text {mat }}(P ; x) \leqslant \frac{10 \pi^{2}}{3} \frac{N}{\log N}+\frac{c_{1} N}{\log ^{2} N}+\frac{c_{2} N}{M \log N}+O\left(N^{1-1 / M} \log ^{2} N\right)$ für alle $x$ aus $A^{\prime}(N)$, und (1) zeigt

$$
\mu(A(N))=O\left(N^{-4 / M} \log N\right)
$$

Wird zunächst statt der gesamten Eolge $(N)_{N \in N}$ nur die Teilfolge $\left(N^{M}\right)_{N \geqslant M}$ betrachtet, so ist

$$
A_{M I}=\bigcup_{N \geqslant M} A\left(N^{M}\right)
$$

die globale Ausnahmemenge; das heißt für alle $N \geqslant \max \left\{M, N\left(M_{0}, c^{\prime}\right)\right\}$ und alle $x \notin A_{M}$ gilt nach (8)

$$
S_{N^{M I}}^{\mathrm{nat}}(P ; x) \frac{\log N^{M I}}{N^{M I}} \leqslant \frac{10}{3} \pi^{2}+O\left(\frac{1}{M I}\right)
$$

und (9) liefert $\mu\left(A_{M I}\right)=O_{f}\left(M^{-1}\right)$. Daraus folgt nach der Methode von Hilfssatz 1

$$
\limsup _{N \rightarrow \infty} S_{N}^{\text {mat }}(P ; x) \frac{\log N}{N} \leqslant \frac{10}{3} \pi^{2}+O\left(\frac{1}{M}\right)
$$

für alle $x \notin A_{M}$, und die zu $O(1 / M)$ gehörige Konstante kann unabhängig von II gewählt werden. Mit $M \rightarrow \infty$ ergibt sich daraus die Behauptung des Satzes.

An entscheidender Stelle im Beweis von Satz 11 wird das folgende Resultat benötigt:

HutFSSATZ 4. (i) Für alle Funktionen $f$ aus $\mathscr{F}_{\text {pol }}$ vom Grad $k \geqslant 2$ ist

$$
\int_{0}^{1} S_{N}^{\text {nat }}(P ; x)^{2} d x=O_{f}\left(\frac{N^{2}}{\log ^{2} N}\right)
$$


(ii) Frür alle $f$ aus $\mathscr{F}_{\exp }$ ist

$$
\int_{0}^{1} S_{N}^{\text {nat }}(P ; x)^{2} d x=O_{f}\left(\log ^{2} N\right)
$$

Beweis. (i) Aus (8) folgt für alle $x$ aus $\mathcal{A}^{\prime}(N)$ bei festem $M$

$$
S_{N}^{\text {nat }}(P ; x)=O\left(\frac{N}{\log N}\right)
$$

und für $x$ ars $A(N)$ ist $S_{N}^{\text {nat }}(P ; \infty) \leqslant N$. Zusammen mit (9) ergibt das

$$
\int_{0}^{1} S_{N}^{\operatorname{mat}}(P ; x)^{2} d x=O_{f}\left(\frac{N^{2}}{\log ^{2} N}+N^{2-4 / M I} \log N\right)=O_{f}\left(\frac{N^{2}}{\log ^{2} N}\right) .
$$

(ii) wird bewiesen ähnlich wie Lemma 3 ; nur werden die einzelnen Summanden rechts in der Gleichung

(10) $\int_{0}^{1} S_{N}^{\text {nat }}(P ; x)^{2} d x=\int_{0}^{1} S_{N}^{\text {nat }}(P ; x) d x+2 \sum_{n=2}^{N} \sum_{m=1}^{n-1} \int_{0}^{1} s_{m}(x) s_{n}(x) d x$

anders abgeschätzt, und zwar mit Selbergs Primzahlformel [28]

$$
\pi_{P}(y+M)-\pi_{P}(y) \leqslant 2 \frac{M}{\log M}+o\left(\frac{M}{\log ^{2} M}\right)
$$

$s_{m}(x)$ nimmt auf $\left|\mathscr{P}_{m}(0,1)\right|$ Intervallen $I_{p, m}^{0,1}$ den Wert 1 an, und auf Grund der Formeln (1) und (2) von Teil 1 ist die Länge jedes dieser Intervalle $o\left(\frac{1}{f^{\prime}(m)}\right) . Z n$ festem $I_{p, m}^{0,1}=[a, b[$ und $n>m$ sind die Intervalle in $[a, b[$, auf denen $s_{n}(x)=1$ ist, genau die $I_{p, n}^{a, b}$ mit $p \in \mathscr{P}_{n}(a, b)$, und deren länge ist jeweils $O\left(\frac{1}{f^{\prime}(n)}\right) \cdot \mathscr{P}_{n}(a, b) \mid$ Iäßt sich mit (11) abschätzen durch

und

$$
\begin{aligned}
\left|\mathscr{P}_{n}(a, b)\right| & =\pi_{P}(f(n+b))-\pi_{P}(f(n+a))+O(x) \\
& =o_{f}\left(\frac{(b-a) f^{\prime}(n)}{\log \left((b-a) f^{\prime}(n)\right)}\right) \\
& =o_{f}\left(\frac{f^{\prime}(n)}{f^{\prime}(m)} \log ^{-1}\left(\frac{f^{\prime}(n)}{f^{\prime}(m)}\right)\right) \\
& =o_{f}\left(\frac{1}{n-m} \frac{f^{\prime}(n)}{f^{\prime}(m)}\right)
\end{aligned}
$$

$$
\begin{aligned}
\left|\mathscr{P}_{m}(0, \mathfrak{1})\right| & =\pi_{P}(f(m+1))-\pi_{P}(f(m))+O(1) \\
& =O_{f}\left(\frac{f^{\prime}(m)}{\log f^{\prime}(m)}\right)=O_{f}\left(\frac{f^{\prime}(m)}{\dot{m}}\right)
\end{aligned}
$$

Zusammen ergibt sich

$$
\int_{0}^{1} s_{m}(x) s_{n}(x) d x=O_{f}\left(\mid \mathscr{P}_{m}(0,1) 1 \frac{1}{n-m}-\frac{f^{\prime}(n)}{f^{\prime}(m)}-\frac{1}{f^{\prime}(n)}\right)=O_{f}\left(\frac{1}{m(n-m)}\right) .
$$

Damit folgt aus (10) und Formel (6) aus Teil 3

$$
\int_{0}^{1} S_{N}^{\text {nat }}(P ; x)^{2} d x=O_{f}\left(\log N+\sum_{n=2}^{N} \sum_{m=1}^{n-1} \frac{1}{m(n-m)}\right)=O_{f}\left(\log ^{2} N\right) .
$$

7. Die Dichte der Primzahlen in Folgen der Form $[f(n+x)]$

SATZ 11. (i) Ist $f$ a aus $\mathscr{F}_{\exp }$ vom Grad $k$, so gilt für die logarithmischharmonische Dichte der Primzahlen in der Folge $[f(n+x)]_{n \in \mathbf{N}}$

$$
D_{\mathrm{har}}^{\log }(P, f)=\frac{1}{\mu} \frac{1}{k} .
$$

(ii). Unter der Voraussestung, daß der Primzahtsatz $\pi_{P}(y)=\operatorname{li} y+$ $+O(\sqrt{y} \log y)$ richtig ist, gilt für die harmonische Dichte der Primzahlen. in $[f(n+x)]_{n \in N}$ für Frunktionen f aus $\mathscr{F}_{\text {pol }}$ vom Grad $k>2$

$$
D_{\mathrm{lar}}^{\mathrm{har}}(P, f)=\frac{1}{\mu} \frac{1}{k} .
$$

Beweis. (i) und (ii) werden simultan gezeigt; dabei kann ohne Einschränkung $f \in \mathscr{F}_{\text {pol }}^{*} \cup \mathscr{F}_{\text {exp }}^{*}$ vorausgesetzt werden.

BEHAUPTUNG 1. Für $T_{1}(y ; x)=\frac{S_{y}^{\text {nat }}(P ; x)}{\Pi(y)}$ und $r=\frac{1}{4} \min \{k-2,2\}$ ist $\int_{0}^{1} T_{1}(M ; x) T_{1}(N ; x) d x \leqslant 1+O_{f}(R(M T, N))$

mit

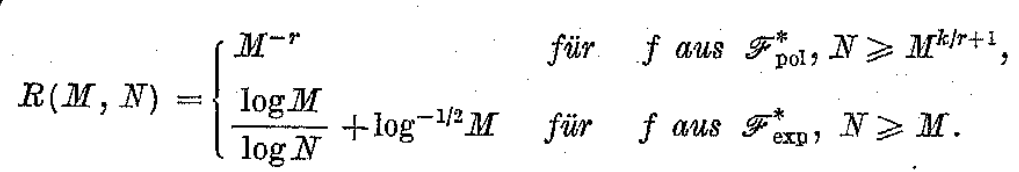

Beweis. Sei zunächst $f$ aus $\mathscr{F}_{\text {po1 }}^{*}$. Mit $\pi_{1}(y)=\operatorname{li} y, \pi_{1}^{\prime}(y)=\log ^{-1} y$ und $\pi_{2}(y)=\sqrt{y} \log y$ lassen sich die $R$-Terme in Lemma 2 vereinfachen zu

Ferner ist

$$
\begin{array}{ll}
R_{21}(N)=O_{f}\left(N^{1-2 r} \log ^{2} N\right), & R_{22}(N)=O_{f}\left(N^{k} \log ^{-1} N\right) \\
R_{23}(N)=O_{f}\left(N^{T / 2+1} \log N\right), & R_{24}(N)=O_{f}(\log \log N)
\end{array}
$$

$$
\Pi(N)=\sum_{n=1}^{N} \frac{1}{\log f(n)}=O_{f}\left(\frac{N}{\log N}\right)
$$


and Lemma 2 ergibt

$$
\begin{aligned}
& \int_{0}^{1} T_{I}(M ; x) T_{1}(N ; x) d x \\
& \begin{aligned}
\leqslant 1+\frac{1}{\Pi(M) \Pi(N)} O_{f}\left(N^{1-2 r} \log ^{2} N\left(\frac{M^{k}}{\log M}+M^{k / 2+1} \log M\right)+\right. \\
\\
\left.+\Pi(N)\left(M^{1-2 r} \log ^{2} M+\log \log M\right)\right)
\end{aligned} \\
& \leqslant 1+O_{f}\left(\frac{\log ^{3} N \log ^{2} M}{N^{-3 r}} M^{k-1}+\frac{\log ^{3} M}{M^{2 r}}\right) \\
& \leqslant 1+O_{f}\left(\frac{M^{k}}{N^{r}}+\frac{1}{M^{r}}\right) \leqslant 1+O_{f}\left(M^{-r}\right) .
\end{aligned}
$$

Für $f$ aus $\mathscr{F}_{\text {exp }}^{*}$ reicht zum Bewreis der Behauptung der Primzahlsatz (PZS 1) mit dem Restglied $\pi_{2}(y)=\frac{y}{\exp \sqrt{\log y}}$. Jetzt ist $\Pi(N)$ $=O_{f}(\log N)$.

Mit der Hölderschen Ungleichung ergibt sich aus Hilfssatz 4(ii)

$$
\int_{0}^{1} S_{M}^{\text {nat }}(P ; x) S_{N}^{\text {mat }}(P ; x) d x=O_{f}(\log M \log N) .
$$

Das beweist den zweiten Teil von Behauptung 1 für $M \leqslant N \leqslant M^{4}$ und liefert zugleich für $N>M^{*}$

(1) $\int_{0}^{1} S_{M}^{\mathrm{nat}}(x) S_{N^{\mathrm{n}}}^{\mathrm{nat}}(x) d x=O_{f}\left(\log ^{2} M\right)+\sum_{m=1}^{M I} \sum_{n=M^{4}+1}^{N} \int_{0}^{1} s_{m}(x) s_{n}(x) d x$.

Aus Formel (5) im Beweis von Lemma 2 folgt mit der Abkürzung $\pi_{3}(y)$ $=\exp \sqrt{\log y}$, wenn $I^{1}$ in $L$ Intervalle gleicher Länge zerlegt wird:

$$
\begin{aligned}
& \int_{0}^{1} s_{m}(x) s_{n}(x) d x-\frac{1}{\log f(m) \log f(n)} \\
& \leqslant O_{f}\left\{\frac{f^{\prime}(m) f(n)}{f^{\prime}(n) \log f(m) \pi_{3} f(n)}+\frac{L f(m)}{f^{\prime}(m) \log f(n) \pi_{3} f(m)}+\right. \\
& \left.+\frac{L f(m) f(n)}{f^{\prime}(n) \pi_{3} f(m) \pi_{3} f(n)}+\frac{f^{\prime \prime}(m)}{L f^{\prime}(m) \log f(m) \log f(n)}\right\} \\
& \leqslant O_{f}\left\{\frac{f(m)}{m \pi_{3} f(n)}+\frac{L}{n \pi_{3} f(m)}+\frac{L f(m)}{\pi_{3} f(m) \pi_{3} f(n)}+\frac{1}{L \log f(m) \log f(n)}\right\} \text {. }
\end{aligned}
$$

Mit

folgt weiter

$$
\sum_{n=M M^{4}+1}^{N} \frac{1}{\pi_{3} f(n)}=O_{f}\left(\frac{M^{3}}{\exp \left(\sqrt{k} M^{2}\right)}\right)
$$

$$
\begin{array}{r}
\sum_{m=1}^{M} \sum_{n=M^{4}+1}^{N} \int_{0}^{1} s_{m}(x) s_{n}(x) d x-\Pi(M) \Pi(N) \leqslant O_{f}\left(\frac{M^{2}}{\exp \left(\sqrt{k} M^{2}-k M\right)}+\right. \\
\left.+L \log N+\frac{L M^{3}}{\exp \left(\sqrt{k} M^{2}-k M\right)}+\frac{1}{L} \Pi(M) \Pi(N)\right) .
\end{array}
$$

Wird das in (1) eingesetzt, ergibt sich für $M \geqslant 3$ und $L=[\sqrt{\log M}]$

$$
\int_{0}^{1} T_{1}(M ; x) T_{1}(N ; x) d x \leqslant 1+O_{f}\left(\frac{\log M}{\log N}+\log ^{-1 / 2} M\right) .
$$

Wis sei $s_{f}=\max \{k / r+1,2\}$. Den nun auftretenden Parameter $s$ wérden wir gegen Ende des Beweises zu einer Funktion von $N$ spezialisieren. Deshalb sollen die folgenden Abschätzungen so vorgenommen werden, daß die dabei in den $O$-Termen auftretenden Konstanten von $s$ unabhängig $\sin d$.

Behauptung 2. Mit den $\mathrm{C}_{1}$-Summen

$$
T_{2}(y, s ; x)=\frac{1}{y} \sum_{0 \leqslant n \leqslant y-1} T_{1}\left(\exp \left(s^{n}\right) ; x\right)
$$

gilt für alle $s \geqslant s_{f}$ und alle natürlichen Zahlen $N$

$$
\int_{0}^{1}\left|T_{2}(N, s ; x)-1\right| d x=O_{f}\left(\frac{1}{\sqrt{N}}\right) .
$$

Beweis. Zunächst ist

$$
\text { (2) } \begin{aligned}
\int_{0}^{1} T_{2}(N, s ; x)^{2} d x= & \frac{1}{N^{2}} \sum_{n=0}^{N-1} \int_{0}^{1} T_{1}\left(\exp \left(s^{n}\right) ; x\right)^{2} d x+ \\
+ & \frac{2}{N^{2}} \sum_{m=0}^{N-2} \sum_{n=m+1}^{N-1} \int_{0}^{1} T_{1}\left(\exp \left(s^{m}\right) ; x\right) T_{1}\left(\exp \left(s^{n}\right) ; x\right) d x
\end{aligned}
$$

Für $f$ aus $\mathscr{F}_{\text {pol }}^{*}$ ist nach Wahl von $s_{f}$ mit $n>m$ stets auch $\exp \left(s^{n}\right)$ $\geqslant\left(\exp \left(s^{m}\right)\right)^{k / r+1}$; Hilfsssatz 4 (i) und Behauptung 1 zeigen also

$$
\int_{0}^{1}\left[T_{2}(N, s ; x)^{2} d x \leqslant 1+o_{f}\left(\frac{1}{N}\right)\right.
$$


Mit Eilfssatz 4(ii), Behauptung 1 und wegen

$$
\sum_{m=0}^{N-2} \sum_{n=m+1}^{N-1}\left(1+o_{f}\left(\frac{s^{m}}{s^{n}}+s^{-m / 2}\right)\right) \leqslant \frac{N^{2}}{2}+o_{f}(N)
$$

folgt (3) aus (2) aber auch für Funktionen aus $\mathscr{F}_{\text {exp }}^{*}$. Ferner ergibt sich

(4) $\quad \int_{0}^{I} T_{2}(N, s ; x) d x=\frac{1}{[N} \sum_{n=0}^{N-1} \int_{0}^{1} \frac{S_{\operatorname{sxp}\left(s^{n}\right)}^{\mathrm{nat}}(P ; x)}{I T\left(\exp \left(s^{n}\right)\right)} d x=1+O_{f}\left(\frac{1}{N}\right)$

für Polynomfunktionen aus Formel (7) in Teil 3 und für Funktionen aus $\mathscr{F}_{\text {exp }}^{*}$ mit Formel (6) aus Teil 3. SchlieBlich zeigen (3) und (4)

$$
\int_{0}^{1}\left|X_{2}(N, s ; \infty)-1\right| d x \leqslant\left(\int_{0}^{1}\left(T_{2}(N, s ; x)-1\right)^{2} d x\right)^{1 / 2}=O_{f}\left(N^{-1 / 2}\right) .
$$

Wir interpretieren nun $T_{2}(N, s ; x)$ als $S_{M}^{\varphi}(P ; x)$ mit passender Wichtung $\psi$ und beginnen, $\psi$ zu vereinfachen:

$$
\begin{aligned}
T_{2}(N, s ; x) & =\frac{1}{N} \sum_{m=0}^{N-1} \frac{S_{\exp \left(s^{m}\right)}^{\text {nat }}(P ; x)}{\Pi\left(\exp \left(s^{m}\right)\right)} \\
& =\frac{1}{N} \sum_{m=0}^{N-1} \sum_{1 \leqslant n \leqslant \exp \left(s^{m}\right)} \frac{\chi_{P}([f(n+x)])}{\Pi\left(\exp \left(s^{m}\right)\right)} \\
& =\frac{1}{N} \sum_{1 \leqslant n \leqslant \exp \left(s^{N-1}\right)} \varphi_{A}(s, n) \chi_{F}([f(n+x)])
\end{aligned}
$$

mit

$$
\varphi_{N}(s, n)=\sum_{\substack{m=0 \\ \exp \left(s^{m}\right) \geqslant n}}^{N-1} \frac{1}{\Pi\left(\exp \left(s^{m}\right)\right)} .
$$

$\varphi_{N}(s ; n)$ wird nun ersetzt durch eine Funktion, die den ersten Summanden der Summe in (6) approximiert. Dazu definieren wir für $s \geqslant s_{f}$

$$
\tau(s, n)=\min \left\{m \in \boldsymbol{N}_{0} ; \exp \left(s^{m}\right) \geqslant n\right\},
$$

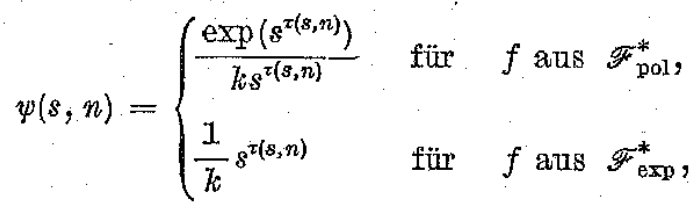

$$
T_{3}(N, s ; x)=\frac{I}{N} \sum_{1 \leqslant n \leqslant \exp \left(s^{N-1}\right)} \frac{\chi_{P}([f(n+x)])}{\psi(s, n)} .
$$

BeHAUPIUNG 3. Für $s \geqslant s_{f}$ und alle natürlichen Zahlen $N$ ist

$$
\int_{0}^{1}\left|T_{3}(N, s ; x)-1\right| d x=O_{f}\left(\frac{1}{\sqrt{N}}+\frac{\log s}{s}\right) .
$$

Beweis. Aus (5) and (8) folgt

(9) $\quad \int_{0}^{1}\left|T_{3}(N, s ; x)-T_{2}(N, s ; x)\right| d x$

$$
\leqslant \frac{1}{N} \sum_{1 \leqslant n \leqslant \exp \left(8^{N-1}\right)}\left|\frac{1}{\psi(s, n)}-\varphi_{N}(s, n)\right| \int_{0}^{1} \chi_{P}([f(n+x)]) d x,
$$

und dabei ist

(10) $\left|\frac{1}{\psi(s, n)}-\varphi_{N}(s, n)\right|$

$$
\leqslant\left|\frac{1}{\psi(s, n)}-\frac{1}{\Pi\left(\exp \left(s^{\tau(s, n)}\right) \mid\right.}\right|+\sum_{m>\tau(s, n)} \frac{1}{\Pi\left(\exp \left(s^{m}\right)\right)} .
$$

Sei zunächst wieder $f$ aus $\mathscr{F}_{\text {pol }}^{*}$. Dann ist $\Pi(y)=O_{f}(y / \log y)$, und der zweite Summand rechts in (10) läßt sich abschätzen durch

$$
\text { (11) } \quad \sum_{m>\tau(s, n)} \frac{1}{\Pi\left(\exp \left(s^{m}\right)\right)}=O_{f}\left(\sum_{m>\tau(s, n} \frac{s^{m}}{\exp \left(s^{m}\right)}\right)=O_{f}\left(\frac{1}{\exp \left(s^{\tau(8, n)}\right)}\right) \text {. }
$$

Ferner ist

$$
\begin{aligned}
\frac{k \log y}{y}-\frac{1}{\Pi(y)} & =\frac{1}{y \Pi(y)}(k(\log y) \Pi(y)-y) \\
& =O_{f}\left(\frac{\log ^{2} y}{y^{2}}\left(\sum_{1 \leqslant n \leqslant y} \frac{k}{\log f(n)}-\frac{y}{\log y}\right)\right) \\
& \left.=O_{f}\left(\frac{\log ^{2} y}{y^{2}}\left(\sum_{1 \leqslant n \leqslant y} \mid \frac{k}{\log f(n)}-\frac{1}{\log ^{2} n}\right)+\frac{y}{\log ^{2} y}\right)\right) \\
& =O_{f}\left(\frac{1}{y}\right) .
\end{aligned}
$$

Daraus folgt für $y=\exp \left(s^{r(s, n)}\right)$

$$
\frac{1}{\psi(s, n)}-\frac{1}{\Pi\left(\exp \left(s^{\tau(s, n)}\right)\right)}=O_{f}\left(\frac{1}{\exp \left(s^{\tau(s, n)}\right)}\right)
$$


Aus den Formeln (9) bis (12) ergibt sich nun

$$
\begin{aligned}
\int_{0}^{1}\left|T_{3}(N, s ; x)-T_{\mathbf{2}}(N, s ; x)\right| d x \\
\quad=o_{f}\left(\frac{1}{N} \sum_{n \geqslant 1} \frac{1}{\exp \left(s^{\tau(s, n)}\right)} \int_{0}^{1} x_{P}([f(n+x)]) d x\right) \\
=O_{f}\left(\frac{1}{N} \sum_{n \geqslant 1} \frac{1}{\exp \left(s^{n}\right)} \sum_{m \leqslant \exp \left(s^{n}\right)} \int_{0}^{1} x_{P}([f(m+x)]) d x\right) \\
=O_{f}\left(\frac{1}{N} \sum_{n \geqslant 1} \frac{1}{\exp \left(s^{n}\right)} \int_{0}^{1} S_{\exp \left(s^{n}\right)}^{\text {nat }}(P ; x) d x\right) \\
=O_{f}\left(\frac{1}{N} \sum_{n \geqslant 1} \frac{1}{s^{n}}\right) \\
=O_{f}\left(\frac{1}{N}\right) .
\end{aligned}
$$

Das zusammen mit Behauptung 2 beweist Behauptung 3 für Funktionen atss $\mathscr{F}_{\mathrm{pol}}^{*}$.

Eür $f$ aus $\mathscr{F}_{\exp }^{*}$ ist $\Pi(y)=O_{f}(\log y)$, und der zweite Summand rechts in (10) läßt sich abschätzen durch

$$
\sum_{m>\tau(s, n)} \frac{1}{\Pi\left(\exp \left(s^{m}\right)\right)}=o_{f}\left(\sum_{m>\tau(s, n)} \frac{1}{s^{m}}\right)=O_{f}\left(\frac{1}{s^{\tau(s, n)+1}}\right)
$$

Außerdem ist

$$
\begin{aligned}
\frac{k}{\log y}-\frac{1}{I I(y)} & =O_{f}\left(\log ^{-2} y\left(\sum_{1 \leqslant n \leqslant y} \frac{k}{\log f(n)}-\log y\right)\right) \\
& =O_{f}\left(\log ^{-2} y\left(\sum_{1 \leqslant n \leqslant y}\left|\frac{k_{i}}{\log f(n)}-\frac{1}{n}\right|+1\right)\right) \\
& =O_{f}\left(\log ^{-2} y\right) .
\end{aligned}
$$

Daraus folgt für $y=\exp \left(s^{\tau(s, n)}\right)$

$$
\frac{1}{\psi(s, n)}-\frac{1}{\Pi\left(\exp \left(s^{2(s, n)}\right)\right)}=O_{f}\left(\frac{1}{s^{2 \pi(s, n)}}\right)
$$

Aus (9), (10), (13) und (14) ergibt sich dann

$$
\text { (15) } \begin{aligned}
& \int_{0}^{1}\left|T_{3}(N, s ; x)-T_{2}(N, s ; x)\right| d x \\
= & \left.O_{f}\left(\left.\frac{1}{N}\right|_{1 \leqslant n \leqslant \exp \left(s^{N-1}\right)}\left(s^{-2 \tau(s, n)}+s^{-\tau(s, n)-1}\right) \int_{0}^{1} \chi_{P}([f(n+x)]] d x\right)\right) \\
= & O_{f}\left(\frac{1}{N}+\frac{1}{s N} \sum_{1 \leqslant n \leqslant \exp \left(s s^{N-1}\right)} s^{-\tau(s, n)} \int_{0}^{1} \chi_{P}([f(n+x)]) d x s\right) \\
= & O_{f}\left(\frac{1}{N}+\frac{1}{s N} \sum_{2 \leqslant n \leqslant \exp \left(s^{N-1}\right)} \int_{0}^{1} \frac{\chi_{P}([f(n+x)])}{\log n} d s\right) \\
= & \left.O_{f}\left(\frac{1}{N}+\frac{1}{s N} \int_{0}^{1} S_{\exp \left(s^{N}-1\right)}^{\log (P ;} ; x\right) d x\right) \\
= & O_{f}\left(\frac{1}{N}+\frac{\log s}{s}\right),
\end{aligned}
$$

denn für $f$ aus $\mathscr{H}_{\text {exp }}^{*}$ folgt mit Lemma 1

$$
\int_{0}^{1} S_{M}^{\log }(P ; x) d x=O_{f}(\log \log M)
$$

Behauptung 2 zusammen mit (15) liefert nun Behauptung 3 auch für Funktionen aus $\mathscr{F}_{\text {exp }}^{*}$.

BeHAUPTUNG 4. Frür $s \geqslant s_{f}$ und $M \geqslant e^{s}$ ist mit

$$
\begin{gathered}
T_{4}(y, s ; x)=\frac{\log s}{\log \log y} \sum_{1 \leqslant n \leqslant 4} \frac{\chi_{p}([f(n+x)])}{\psi(s, n)} \\
\int_{0}^{1}\left|T_{4}(M, s ; x)-1\right| d x=O_{f}\left(\left(\frac{\log s}{\log \log M}\right)^{1 / 2}+\frac{\log s}{s}\right) .
\end{gathered}
$$

Beweis. Für $N \geqslant 1$ und $\exp \left(s^{N}\right) \leqslant M<\exp \left(s^{N+1}\right)$ ist

$$
\begin{gathered}
x_{4}(M, s ; x) \leqslant \frac{\log s}{\log \left(s^{N}\right)} \sum_{n \leqslant \exp \left(8^{N+1}\right)} \frac{\chi_{P}([f(n+\infty)])}{\psi(s, n)}=\left(1+\frac{2}{N}\right) T_{3}(N+2, s ; \infty), \\
T_{4}(M, s ; x) \geqslant \frac{\log s}{\log \left(s^{N+1}\right)} \sum_{n \leqslant \exp \left(s^{N}\right)} \frac{\chi_{P}([f(n+x)])}{\psi(s, n)}=T_{3}(N+1, s ; x)
\end{gathered}
$$


Daraus folgt

$$
\begin{aligned}
|| T_{3}(M, s ; x)-1|-| T_{3}(N+1, s ; x)-1|| \\
\quad \leqslant\left|T_{4}(N, s ; x)-T_{3}(N+1, s ; x)\right| \\
\quad \leqslant\left(1+\frac{2}{N}\right) T_{3}(N+2, s ; x)-T_{3}(N+1, s ; x) \\
\quad \leqslant\left(1+\frac{2}{N}\right)\left|T_{3}(N+2, s ; x)-1\right|+\left|T_{3}(N+1, s ; x)-1\right|+\frac{2}{N} .
\end{aligned}
$$

Integration liefert mit Behauptung 3

$$
\int_{0}^{1}\left|T_{4}(M, s ; x)-1\right| d x=O_{f}\left(\frac{1}{\sqrt{N}}+\frac{\log s}{s}\right)
$$

and aus $M<\exp \left(s^{N+1}\right)$ folgt $\frac{1}{N+1}<\frac{\log s}{\log \log M}$.

Im nächsten Schritt wird die Wichtungsfunktion $\psi(s, n)$ von $T_{4}$ geglättet:

BeHAUPTUNG 5. Fiür $s \geqslant s_{f}$ und $N \geqslant e^{s}$ ist mit

$$
\begin{aligned}
& T_{5}(y, s ; x)=\int_{s}^{s+1} T_{4}(y, t ; x) d t \\
&=\frac{1}{\log \log y} \sum_{1 \leqslant n \leqslant y} \int_{s}^{s+1} \frac{\log t}{\psi(t, n)} d t \chi_{P}([f(n+x)]), \\
& \int_{0}^{1}\left|T_{5}(N, s ; x)-1\right| d x=O_{f}\left(\left(\frac{\log s}{\log \log N}\right)^{1 / 2}+\frac{\log s}{s}\right) .
\end{aligned}
$$

Beweis. Auf Grund von Behauptung 4 ist

$$
\begin{aligned}
\int_{0}^{1}\left|T_{5}(N, s ; x)-1\right| d x & =\int_{0}^{1}\left|\int_{s}^{s+1} T_{4}(N, t ; x) d t-1\right| d x \\
& \leqslant \int_{0}^{1} \int_{s}^{s+1}\left|T_{4}(N, t ; x)-1\right| d t d x \\
& =\int_{s}^{s+1} \int_{0}^{1}\left|T_{4}(N, t ; x)-1\right| d x d t \\
& =\int_{s}^{s+1} o_{f}\left(\left(\frac{\log t}{\log \log N}\right)^{1 / 2}+\frac{\log t}{t}\right) d t \\
& =o_{f}\left(\left(\frac{\log s}{\log \log N}\right)^{1 / 2}+\frac{\log s}{s}\right)
\end{aligned}
$$

BeHAUPTUNG 6. Die Wichtungsfanktion von $T_{5}$ täßt sich abschätzen dureh

$\int_{s}^{s+1} \frac{\log t}{\psi(t, n)} d t=\left\{\begin{array}{lr}\frac{k}{n}+O_{f}\left(\frac{1}{s n}+\frac{s \log ^{2} s}{n \log \log n}\right), & f \in \mathscr{F}_{\text {pol }}^{*}, n \geqslant 3, \\ \frac{\pi}{\log n}+O_{f}\left(\frac{1}{s \log n}+\frac{s \log ^{2} s}{\log n \log \log n}\right), & f \in \mathscr{F}_{\exp }^{*}, n \geqslant 3, \\ O_{f}(\log s), & n<3 .\end{array}\right.$

Beweis. Fïr $n<3$ ist $\tau(t, n)=0$, und aus der Definition von $\psi$ in Formel $(7)$ folgt $\psi(t, n)=O_{f}(1)$. Sei also $n \geqslant 3 . \tau(t, n)$ ist monoton fallende Funktion in $t$ von $] 1, \infty\left[\right.$ in die natürlichen Zahlen. Mit $t_{0}=\infty$ und $t_{m}=(\log \eta)^{1 / m}$ für $m$ aus $\boldsymbol{N}$ ist $\tau(t, n)=m$ genau für die $t$ ans $\left[t_{m}, t_{m-1}[\right.$. $\mathrm{Zu}$ vorgegebenem $s \geqslant s_{f}$ gilt

$$
\begin{gathered}
t_{j+1} \leqslant s<t_{j}<\ldots<t_{i+1}<s+1 \leqslant t_{i}, \\
\frac{\log \log n}{\log s}-1 \leqslant j<\frac{\log \log n}{\log s}, \\
\frac{\log \log n}{\log (s+1)}-1<i \leqslant \frac{\log \log n}{\log (s+1)} .
\end{gathered}
$$

Wegen $\sum_{v \leqslant y} 1 / v=\log y+\gamma+O(1 / y)$ folgt daraus insbesondere für $\delta=0$ und $\delta=1$

$$
\sum_{u=i+1}^{j-1} \frac{1}{v+\delta}=\frac{1}{(s+\theta) \log (s+\theta)}+O\left(\frac{\log s}{\log \log n}\right)
$$

Mit

wird

$$
J_{v}=\int_{t_{v+1}}^{t_{v}} \frac{\log t}{\psi(t, n)} d t, \quad J_{v}^{\prime}=\int_{\max \left\{t_{v+1}, s\right\}}^{\min \left\{t_{v} s+1\right\}} \frac{\log t}{\psi(t, n)} d t
$$

$$
\int_{s}^{s+1} \frac{\log t}{\psi(t, n)} d t=\sum_{v=i+1}^{j-1} J_{v}+O\left(J_{i}^{\prime}+J_{j}^{\prime}\right)
$$

Für $f$ aus $\mathscr{F}_{\text {pol }}^{*}$ and $i<v<j$ ist

$$
\begin{aligned}
J_{v} & =k \int_{t_{v+1}}^{t_{v}} t \log t \frac{t^{v}}{\exp \left(t^{v+1}\right)} d t \\
& =\frac{l_{v}}{v+1}\left(s+\Theta_{v}\right) \log \left(s+\Theta_{v}\right)\left(\frac{1}{n}-\left(\frac{1}{n}\right)^{t_{v}}\right) \\
& =\frac{k}{n(v+1)} s \log s\left(1+o\left(\frac{1}{s}\right)\right),
\end{aligned}
$$

s-Acta Arithmetica $\mathrm{xxxV.2}$ 
und mit (16) folgt

$$
J_{i}^{\prime}+J_{j}^{\prime} \leqslant \frac{2 \hbar}{n(i+1)}(s+1) \log (s+1)=O_{f}\left(\frac{s \log ^{2} s}{n \log \log n}\right) .
$$

Daraus ergibt sich mit (18) und (1i)

$$
\begin{aligned}
\int_{s}^{s+1} \frac{\log t}{\psi(t, n)} d t & =\frac{k}{n} s \log s\left(1+O\left(\frac{1}{s}\right)\right) \sum_{n=i+1}^{j-1} \frac{1}{v+1}+O_{f}\left(\frac{s \log ^{2} s}{n \log \log n}\right) \\
& =\frac{k}{n} s \log s\left(1+O\left(\frac{1}{s}\right)\right) \frac{1}{(s+\Theta) \log (s+\Theta)}+O_{f}\left(\frac{s \log ^{2} s}{n \log \log n}\right) \\
& =\frac{k}{n}+O_{f}\left(\frac{1}{s n}+\frac{s \log ^{2} s}{n \log \log n}\right)
\end{aligned}
$$

Für $f$ aus $\mathscr{F}_{\exp }^{*}$ und $i<v<j$ wird.

$$
\begin{aligned}
J_{v} & =k \int_{t_{v+1}}^{t_{v}} \frac{\log t}{t^{v+1}} d t \\
& =\frac{k}{v} \log \left(s+\Theta_{v}\right) \frac{1}{\log n}\left(t_{v+1}-1\right) \\
& =\frac{k}{v \log n} s \log s\left(1+O\left(\frac{1}{s}\right)\right)
\end{aligned}
$$

mit (16) folgt für $i>0$

$$
J_{i}^{t}+J_{j}^{t} \leqslant \frac{2 k}{i \log n} s \log (s+1)=O_{f}\left(\frac{s \log ^{2} s}{\log n \log \log n}\right),
$$

und im Fall $i=0$ ist nach (16) $\log n<s+1$ und deshalb

$$
J_{0}^{\prime} \leqslant \hbar \int_{s}^{s+1} \frac{\log t}{t} d t=O_{f}\left(\frac{\log ^{2} s}{\log n \log \log n}\right) .
$$

Daraus folgt mit (18) und (17)

$$
\begin{aligned}
& \int_{s}^{s+1} \frac{\log t}{\psi(t, n)} d t=\frac{k}{\log n} s \log s\left(1+O\left(\frac{1}{s}\right)\right) \sum_{v=i+1}^{j-1} \frac{1}{v}+O_{f}\left(\frac{s \log ^{2} s}{\log n \log \log n}\right) \\
& =\frac{k}{\log n} s \log s\left(1+O\left(\frac{1}{s}\right)\right) \frac{1}{(s+\Theta) \log (s+\Theta)}+O_{f}\left(\frac{s \log ^{2} s}{\log n \log \log n}\right) \\
& =\frac{k}{\log n}+O_{f}\left(\frac{1}{s \log n}+\frac{s \log 2}{\log n \log \log n}\right) .
\end{aligned}
$$

Das beweist Behauptung 6 .
BEHATPTUNG 7 . Mit

und

$$
\psi(y)=\left\{\begin{array}{lll}
y & \text { für } & f \text { aus } \mathscr{F}_{\text {poi }}^{*}, \\
\max \{\log 2, \log y\} & \text { für } & f \text { aus } \mathscr{F}_{\text {exp }}^{*}
\end{array}\right.
$$

$$
T_{6}(N ; x)=\frac{k}{\log \log N} S_{N}^{w}(P ; x)
$$

giltfür $N \geqslant N_{f}=\exp \exp \left(s_{f}^{2}\right)$

$$
\int_{0}^{1}\left|T_{6}(N ; x)-1\right| d x=O_{f}\left(\frac{(\log \log \log N)^{2}}{\sqrt{\log \log N}}\right) .
$$

Beweis. Wir wählen $s=s(N)=\sqrt{\log \log N}$. Aus $N \geqslant N_{f}$ folgt dann $s(N) \geqslant s_{f}$. Außerdem ist stets $N \geqslant e^{s(N)}$. Behauptung 5 liefert also

$$
\int_{0}^{1}\left|T_{5}(N, s(N) ; x)-1\right| d x=O_{f}\left(\frac{\log \log \log N}{\sqrt{\log \log N}}\right) .
$$

Aus Behauptung 6 kommt

(20) $\left|T_{6}(N ; x)-T_{5}(N, s(N) ; x)\right|$

$$
\begin{aligned}
& =\frac{1}{\log \log N}\left|\sum_{n=1}^{N}\left(\frac{k}{\psi(n)}-\int_{s(N)}^{s(N)+1} \frac{\log t}{\psi(n, t)} d t\right) \chi_{P}([f(n+x)])\right| \\
& =\frac{1}{\log \log N} O_{f}\left(\log s(N)+\sum_{n=3}^{N}\left(\frac{1}{s(N) \psi(n)}+\frac{s(N) \log ^{2} s(N)}{\psi(n) \log \log n}\right) \chi_{P}([f(n+x)])\right) \\
& =O_{f}\left(\frac{\log \log \log N}{\log \log N}+\frac{1}{\log \log N}\left(\frac{S_{N}^{p}(P ; x)}{s(N)}+s(N) \log ^{2} s(N) S_{N}^{\hat{\varphi}}(P ; x)\right)\right) .
\end{aligned}
$$

mit

$$
\hat{\psi}(y)=\max \{\psi(3) \log \log 3, \psi(y) \log \log y\} .
$$

Gemäß Lemma 1 ist

$$
\int_{0}^{1} S_{N}^{\varphi}(P ; x) d x=O_{f}(\log \log N), \quad \int_{0}^{1} S_{N}^{\hat{\varphi}}(P ; x) d x=O_{f}(\log \log \log N) .
$$

Damit folgt ans (20)

$$
\int_{0}^{1}\left|T_{6}(N ; x)-T_{5}(N, s(N) ; x)\right| d x=O_{f}\left(\frac{(\log \log \log N)^{3}}{\sqrt{\log \log N}}\right)
$$

und das zusammen mit (19) zeigt Behauptung 7 . 
Für $g(N)=\left[\exp \exp \left(N^{4}\right)\right]$ ist

$$
\frac{(\log \log \log g(N))^{3}}{\sqrt{\log \log g(N)}}=O\left(\frac{\log ^{3} N}{N^{2}}\right)
$$

und summiert über $N$ ist die Summe der 0 -Terme rechts in (21) konvergent. Aus Behauptung 7 ergibt sich demnach

$$
\lim _{N \rightarrow \infty} \frac{1}{\log \log g(N)} S_{g(N)}^{\varphi}(P ; x)=\frac{1}{\mu} .
$$

Mit $h(N)=\log \log N$ zeigt Hilfssatz 1 nun

$$
\lim _{N \rightarrow \infty} \frac{1}{\log \log N} S_{N}^{\text {i }}(P ; x)=\frac{1}{\bar{\mu}}
$$

tund daraus schließlich folgen wegen

$$
\sum_{n=1}^{N} \frac{\chi_{P}(n)}{n} \sim \log \log N
$$

die beiden Aussagen von Satz 11.

CoRoLLAR. Mit $f_{c}(y)=c y^{k}+d, k \geqslant 1$, stellen für fast alle $c>0$ die Folgen $\left[f_{c}(n)\right]_{n \in N}$ je unendlich viele Primzahlen dar.

Beweis. Für ganzzahliges $m$ und $f_{m}^{*}(y)=e^{k(y+m) \log 2}+d$ ist

$$
f_{m}^{*}(n+\infty)=f_{c}\left(2^{n}\right) \quad \text { mit } \quad c=2^{k(m+\infty)} .
$$

Nach Satz 11(i) gilt also die Behauptung des Oorollars zunächst für die Koefizienten $c$ ans dem Intervall $\left[2^{k m}, 2^{k(m+1)}[\right.$, and die Vereinigung dieser Intervalle für $m$ aus $\boldsymbol{Z}$ ist $\boldsymbol{R}_{+}-\{0\}$.

Bemerkangen. (1) Satz 11 zeigt insbesondere, daß die Mengen

$$
A_{f}=\left\{x \in I^{1} ; \sum_{\substack{n \in N \\[f(n+x)] \in P}} 1<\infty\right\}, \quad f \text { aus } \mathscr{F}_{\text {pol }} \cup \mathscr{F}_{\exp }
$$

Lebesgue-Maß 0 haben - für $f$ aus $\mathscr{F}_{\text {pol }}$ unter der unbewiesenen Voraussetzung, daß der Primzahlsatz (PZS2) richtig ist. Ohne Benutzung von (PZS2) läBt sieh zeigen, da $B$ für alle $f$ aus $\mathscr{F}_{\text {pol }}$ vom Grad $k>12 / 5$ und alle $f$ aus $\mathscr{F}_{\text {exp }} A_{f}$ von erster Kategorie ist.

Dazu sei für festes $f$

$$
A_{m}=\left\{x \in I^{1} ; \sum_{[f(n+x)] \in P} 1 \leqslant{ }_{i}^{*} m\right\}, \quad B_{m}=I^{1}-A_{m} .
$$

In den Bezeichungen von Teil 1 besagt $x \in B_{m}, \mathrm{da} B m+1$ verschiedene Intervalle $I_{p_{v}, n_{v}}^{0,1}$ exjstieren, deren Durchselinitit $x$ enthält. $B_{m}$ ist demnach abzählbare Vereinigung halboffener Intervalle $J_{\text {, in }} I^{1}$. Dann ist $B_{n}^{*}=\bigcup_{t} \dot{J}_{i}$ offen und $A_{m}^{*}=I^{1}-B_{m}^{*}$ abgeschlossen in $I^{1}$. Mit Satz 1 und dem sich anschließenden Corollar folgt, da $\beta A_{m}^{*}$ nirgends dicht ist. Also ist $\bigcup_{m \geqslant 0} A_{m}^{*}$ von erster Kategorie und wegen $A_{m} \subset A_{m}^{*}$ dann auch $A_{f}=\bigcup_{m \geqslant 0} A_{m}$.

(2) Die glatte Formulierung von Satz 11 verschleiert, wie wild sich die asymptotischen Dichtefunktionen

$$
\bar{D}(x)=\limsup _{N \rightarrow \infty} D(P, f ; N)(x), \quad \underline{D}(x)=\liminf _{N \rightarrow \infty} D(P, f ; N)(x)
$$

gebärden können. Nit $D=D_{\text {har }}^{\text {har }}$ für $f$ aus $\mathscr{F}_{\text {poI }}$ vom Grad $k>2$ und $D=D_{\text {har }}^{\log }$ für $f$ an.s $\mathscr{F}$ exp läßt sich zeigen:

(i) Ist die Menge $\underline{B}(a)$ aller $x$ aus $I^{1}$ mit $\underline{D}(x) \leqslant$ a dioht in $I^{1}$, so gilt mit einer Menge $\underline{K}(a)$ erster Kategorie

$$
\underline{B}(a)=I^{1}-\underline{K}(a) .
$$

(ii) Ist alie Menge $\bar{B}(a)$ aller $x$ aus $I^{1}$ mit $\tilde{D}(x) \geqslant a$ aicht in $I^{1}$, so gilt mit einer Menge $\bar{K}(a)$ erster Kategorie

$$
\bar{B}(a)=I^{1}-\bar{K}(a)
$$

Mit $K^{n, N}=\left\{x \in I^{1} ; D(P, f ; M)(x)>a+1 / n\right.$ für alle $\left.M \geqslant N\right\}$ ist

$$
\underline{K}(a) \subset \bigcup_{n, N \in \mathbb{N}} K^{n, N} \text {. }
$$

Zum Nachweis von (i) bleibt also nur einzusehen, daß bei dichtem $B(a)$ jedes $K^{n, N}$ nirgends dicht ist. Angenommen, es gäbe ein Intervall $I$ in $I^{1}$, bezüglich dessen $K^{n, N}$ dicht ist, so existierten nach der Voraussetzung über $\underline{B}(a)$ ein $x_{0}$ in $\underline{B}(a) \cap I$ und ein $N^{\prime} \geqslant N$ mit $D\left(P, f ; N^{\prime}\right)\left(x_{0}\right)<a+1 / n$. Da $\underline{D}\left(P, f ; N^{\prime}\right)(\infty)$ Treppenfunktion ist, gäbe es ein $x_{0}$ enthaltendes Intervall $I^{\prime}$ in $I$, auf dem $D\left(P, f ; N^{\prime}\right)$ konstant und $<a+1 / n$ wäre. Das aber widerspricht der Annahme. Ahnlich läßt sich (ii) beweisen.

(3) Zur Illustration von Bemerkung 2 sei

$$
f_{m}(y)=m^{y}, \quad m \in \mathbf{N}, m \geqslant 2 .
$$

$\bar{B}(1 / \log m)$ ist dicht in $I^{1}$ nach Satz $11(i)$, and auch $\underline{B}(0)$ ist dicht, weil es die Bruchteile aller Zahlen $\log n / \log m$ für $n$ aus $N$ enthält. Das zeigt:

Frïr $f_{m}$ gilt außerhalb einer Mrenge erster Kategorie stets zugleioh

$$
\underline{D}_{\mathrm{har}}^{\log }\left(P, f_{m}\right)(x)=0 \quad \text { und } \quad \breve{D}_{\mathrm{har}}^{\log }\left(P, f_{m}\right)(x) \geqslant \frac{1}{\log m}
$$

(4) Historische Hinweise über Vermutungen zur Frage der Primahlverteilung bei ganzwertigen nichtlinearen Polynomfunktionen finden sich bei Halberstam-Richert [9], S. 9-11. Deshalb sei hier nur auf eine 
Spezialisierung der Hypothese $\mathrm{H}^{*}$ von Bateman-Horn [2] hingewiesen, die Vermutungen von Hardy-Littlewood [11] über die Anzahl der Primzahlen in den Folgen $\left(m^{2}+1\right)_{m \in N}$ (Conjecture $\left.\mathrm{E}\right),\left(a m^{2}+b m+c\right)_{m \in N}$ (Conjecture $\mathrm{F}$ ) und $\left(m b^{3}+k\right)_{m \in \boldsymbol{N}}$ (Conjecture K) verallgemeinert:

Ist $f$ eine über $Q$ irreduzible Polynomfunktion mit ganzen Koeffizienten und positivem Leitkoeffizienten, und bezeichnet $\varrho(p)$ für Primzahlen $p$ die Anzahl der Lösungen ron $f(n) \equiv 0 \bmod p$, dann gilt für die Anzahl $S_{X^{*}}(P, f)$ der Primzahlen in der Folge $\left(\left.f(n)\right|_{1 \leqslant n \leqslant N}\right.$

$$
\lim _{N \rightarrow \infty} \frac{S_{N}(P, f)}{\operatorname{li} N}=\frac{1}{\operatorname{deg} f} \prod_{p} \frac{p-\varrho(p)}{p-1} .
$$

Aus dieser Hypothese folgt mit Abelscher partieller Summation beispielsweise $D_{\text {har }}^{\text {har }}\left(P, y^{4}+1\right)(0)=0,66974 \ldots$ (Shanks [29]).

(5) Gillies [8] vermutet, daß für die Anzahl $S_{N}$ der Primzahlen unter den ersten $N$ Mersenne-Zahlen $2^{n}-1,1 \leqslant n \leqslant N$, die asymptotische Formel

$$
\lim _{N \rightarrow \infty} \frac{S_{N}}{\log N}=\frac{2}{\log 2}
$$

richtig ist. Für $D_{N}^{*}=(\log 2) D_{\mathrm{har}}^{\log }\left(P, 2^{y}-1 ; N\right)(0)$ würde daraus $\lim _{N \rightarrow \infty} D_{N}^{*}=2$ folgen. Aus den derzeitigen Kenntnissen über Mersennesche Primzahlen

\begin{tabular}{|c|c|c|c|c|c|c|c|c|c|}
\hline & $N-$ & $S$ & $O_{N-1}^{*}$ & $D_{N}^{*}$ & $N$ & $S_{N-1}$ & $S_{N}$ & $D_{N-1}^{*}$ & $D_{N}^{*}$ \\
\hline 2 & 0 & 1 & & 2,00 & 521 & 12 & 13 & 1,90 & 1,95 \\
\hline 3 & 1 & 2 & 2,00 & 1,96 & 607 & 1.3 & 14 & 1,93 & 1,98 \\
\hline$\tilde{5}$ & 2 & 3 & 1,96 & 2,00 & 1279 & 14 & 15 & 1,88 & 1,93 \\
\hline 7 & 3 & 4 & 2,00 & 2,06 & 2203 & 1 ฮั & 16 & 1,87 & 1,91 \\
\hline 13 & 4 & 5 & 1,91 & 2,00 & 2281 & 16 & 17 & 1,90 & 1,94 \\
\hline 17 & 5 & 6 & 2,00 & 2,09 & 3217 & 17 & 18 & 1,90 & 1,94 \\
\hline 19 & 6 & 7 & 2,09 & 2,18 & 4253 & 18 & 19 & 1,91 & 1,95 \\
\hline 31 & 7 & 8 & 2,07 & 2,15 & 4423 & 19 & 20 & 1,95 & 1,98 \\
\hline 61 & 8 & 9 & 1,99 & 2,06 & 9689 & 20 & 21 & 1,91 & 1,94 \\
\hline 30 & 9 & 10 & 1,99 & 2,06 & 9941 & 21 & 22 & 1,94 & 1,97 \\
\hline & 10 & 11 & 2,03 & 2,10 & 11213 & 22 & 23 & 1,96 & 1,99 \\
\hline & 11 & 12 & 2,08 & 2,14 & 19937 & 23 & 24 & 1,94 & 1,97 \\
\hline
\end{tabular}
ergibt sich die folgende Tabelle:

(6) Sollte sich dereinst herausstellen, daß $x_{0}=0$ nicht der Nullmenge angehört, für die $D_{\text {har }}^{\mathrm{Lgg}}\left(P, 2^{y}+1\right)(x)=1 / \log 2$ falsch ist, so würde das bedenten, daß Fermat mit seiner Vermutung, alle Zahlen der Gestalt $2^{2^{n}}+1$ seien Primzahlen, ,im wesentlichen" recht hatte. Denn $D_{\text {hax }}^{\log }\left(P, 2^{y}+1\right)(0)$
$=1 / \log 2$ besagt

$$
\sum_{n=1}^{N} \frac{1}{n} x_{P}\left(2^{2^{n}}+1\right) \sim \sum_{n=1}^{N} \frac{1}{n} .
$$

Jedoch zeigt ein Vergleich mit Corollar 2 zu Satz 3 sofort die ganze Problematik derartiger Spekulationen.

\section{Literaturverzeichnis}

[1] A. R. Ansari, On prime representing functions, Ganita 2 (1931), S. 81-82.

[2] P. T. Bateman and R. A. Horn, A heuristic asymptotic formula concerning the distribution of prime mumbers, Math. Comp. 16 (1962), \$. 363-367.

[3] H. Cram on the order of magnitude of the difference between consecutive prime numbers, Acta Arith. 2 (1937), S. 23-46.

[4] T. Darkicham, Chicago 1967.

[4] arithmétiques, C. R. Acad. Sc. Paris A-B 277 (1973), S. A647-A650.

arithmétiques, C. R. Acad. Sc. Paris A-B 277 (1973), S. A6th. Monthly 76 (1969), S. $23-28$.

77] $\mathrm{E}$. . The Tauk Latvijskoj SSR 6 (1948), S. 97-135.

8] Als (1964), S. 93-97.

9 New York - San Francisco 1974

[10]

11i] G. H. Harthe 44 (1923) S. $1-70$.

[12] G. Wright, An introduction to the theory of numbers, 4. Aufl., Clarendon Press, Oxford 1960.

17. An the difference between consecutive primes, Inventiones math. I5 (1972), S. 164-170.

[14] (Oxford) 8 (1937), S. $255-266$.

[15] Math. Z. 18 (1923), S. 289-306.

[16] J. F. Koksma, Diophantische Approximationen, Springer, Berlin 1936.

[17] Zametki 2 (1967), S. 117-128.

[18] S. $57-58$

110] Tolke, Primzahlen der Gestali $\left[n^{T}\right]$ in arithmetischen Progressionen, Arch. Math. 25 (1974), S. 492-494.

[20] - - Prim:ahlen der Gesialt [ $f(n)]$, Math. Z. 145 (1975), S. 81-92. 53 (1947),

21] W. H. Mills, A prine-representing function, Bull. Amee S. 604 .

[22] (1951), s. $753-755$. 
[23] H.-F. Ostmann, Additive Zahlentheorie I, Springer, Berlin-GöttingenHeidelberg 1956.

[24] A. Ostrowski, Bemerkungen sur Theorie der Diophantischen Approximationen $I, I I, I I I$, Abh. Math. Sem. Hamburg 1 (1922), S. 77-98, 250-251; 4 (1926), S. 224 .

[25] I. I. Pjateckii-Shapiro, Über die Terteilung von Primzahlen in Folgen der Form $[f(n)]$, Math. Sb. 33 (1953), S. 559-566.

[26] G. J. Rieger, Über die natürlichen und primen Zahlen der Gestalt $\left[n^{c}\right]$ in arithmetischer Progression, Arch. Math. I8 (1967), S. 35-44.

[27] W. Sohwarz, Einführung in Siebmethoden der analytischen Zahlentheorie, Bibliographisches Institut, Nannheim -Wien-Zürich 1974.

[28] A. Selberg, On elementary methods in prime number theory and their limitations, In: Den 11-te Skandinaviske Ifatematiker7vongress, Trondheim 1949, Johan Grundt Tanums Forlag, Oslo 1952; S. 13-22.

[29] D. Shanks, On numbers of the form $n^{4}+1$, Math. Comp. 15 (1961), S. 186-189.

[30] D. Wolke, Multiplitative Funfetionen auf schnell wachsenden Folgen, J. Reine Angew. Math. 251 (1971), S. 54-67.

[31] E. M. Wright, A class of representing functions, J. London Math. Soc, 29 (1954), S. $63-71$.

[32] J.-M. Deshouillers, Nombres premiers de la forme [nc], C. R. Acad. Sci. Paris 282 (1976), S. A131-A133.

[33] D. Nordon, Nombres premiers de la forme [un²], Arch. Math. 28 (1977), S. $395-400$.

\section{О6 осковных едншщах алтебраических полей $n$-го порядка.}

\author{
Э. Т. Аванесов (Кисловодск)
}

Рассмотрим диофантово уравнение

$$
x^{n}+\sum_{i=1}^{n} p_{i} x^{n-i} y^{i}=1
$$

где $n \geqslant 3, p_{i}(i=1,2, \ldots, n)$ - целые рациональные числа.

На основании известных результатов Бейкера [1] для возможных целых рештений $(x, y)$ уравнения (1) справедлива следующая оценка

$$
\max (|x|,|y|)<\exp \left(n^{v^{2}} M^{p n^{3}}\right) \text {, }
$$

где $v=32 n \tau^{2} /(\tau-n-1), \tau>n+1-$ пропзвольно, $M=\max _{i}\left|p_{i}\right|_{\text {. }}$

С алгебраической точки зрения репение уравнения (1) сводится к разысканию в кольце $O(\lambda)$, где $\lambda$ - произвольный корень неприводимого уравнения

$$
\lambda^{n}=p_{1} \lambda^{n-1}-p_{2} \lambda^{n-2}+\ldots+(-1)^{n-2} p_{n-1} \lambda+(-1)^{n-1} p_{n},
$$

имеющего пискриминант $D_{k}$, единиц специального двучленного вида $\varepsilon=x+y \lambda$.

Без ограничения общности можно рассматривать кольцо $O(\lambda)$ со стетенным базисом $\left\{1, \lambda, \lambda^{2}, \ldots, \lambda^{n-1}\right\}$.

Тогда в силу теоремы Дирихле для любой едпницы $\varepsilon=x_{0}+x_{1} \lambda+$ $+\ldots+x_{n-1} \lambda^{n-1}$ кольца $O(\lambda)$

$$
\varepsilon=\zeta \varepsilon_{1}^{\alpha_{1}} \varepsilon_{2}^{a_{2}} \ldots \varepsilon_{s+t-1}^{\alpha_{s+t-1}},
$$

где $\zeta$ - некоторый содержащийся в $O(\lambda)$ норень из $1, s$ - число вещественғых коорней, $t$ - число пар комплексных норней уравнения (2); $n=s+2 t$, все $x_{i}-$ целые, $\alpha_{i}(i=1,2, \ldots, s+t-1)$ - пропзвольные целые рациональные цисла, $\varepsilon_{i}(i=1,2, \ldots, s+t-1)$ составляют систему основныг единиц кольца $O(\lambda)$.

Тағим образом задача нахождения целых решений (1) или двучленных единиц $O(\lambda)$, что одно и то же с алгебрапческой точки зрения, опирается на определение основных единиц этого же кольца. 